\title{
A Neyman-Pearson Approach to Universal Erasure and List Decoding
}

\author{
Pierre Moulin, Fellow, IEEE
}

\begin{abstract}
When information is to be transmitted over an unknown, possibly unreliable channel, an erasure option at the decoder is desirable. Using constant-composition random codes, we propose a generalization of Csiszár and Körner's maximum mutual information (MMI) decoder with an erasure option for discrete memoryless channels. The new decoder is parameterized by a weighting function that is designed to optimize the fundamental tradeoff between undetected-error and erasure exponents for a compound class of channels. The class of weighting functions may be further enlarged to optimize a similar tradeoff for list decoders-in that case, undetected-error probability is replaced with average number of incorrect messages in the list. Explicit solutions are identified.

The optimal exponents admit simple expressions in terms of the sphere-packing exponent, at all rates below capacity. For small erasure exponents, these expressions coincide with those derived by Forney (1968) for symmetric channels, using maximum a posteriori decoding. Thus, for those channels at least, ignorance of the channel law is inconsequential. Conditions for optimality of the Csiszár-Körner rule and of the simpler empirical-mutual-information thresholding rule are identified. The error exponents are evaluated numerically for the binary symmetric channel.
\end{abstract}

Index Terms-Constant-composition codes, erasures, error exponents, list decoding, maximum mutual information (MMI) decoder, method of types, Neyman-Pearson hypothesis testing, random codes, sphere packing, universal decoding.

\section{INTRODUCTION}

$\mathbf{U}$ NIVERSAL decoders have been studied extensively in the information theory literature as they are applicable to a variety of communication problems where the channel is partly or even completely unknown [1], [2]. In particular, the maximum mutual information (MMI) decoder provides universally attainable error exponents for random constant-composition codes over discrete memoryless channels (DMCs). In some cases, incomplete knowledge of the channel law is inconsequential as the resulting error exponents are the same as those for

Manuscript received January 29, 2008; revised April 13, 2009. Current version published September 23, 2009. This work was supported by the National Science Foundation (NSF) under Grant CCF 06-35137 and by DARPA under the ITMANET program. The material in this paper was presented in part at the IEEE International Symposium on Information Theory (ISIT), Toronto, ON, Canada, July 2008.

The author is with the Electrical and Computer Engineering Department, Beckman Institute, and the Coordinated Science Laboratory, University of Illinois at Urbana-Champaign, Urbana, IL 61801 USA (e-mail: moulin@ifp.uiuc. edu).

Communicated by H. Yamamoto, Associate Editor for Shannon Theory.

Color versions of Figures 1, 2, and 4 in this paper are available online at http://ieeexplore.ieee.org.

Digital Object Identifier 10.1109/TIT.2009.2027569 maximum-likelihood decoders which know the channel law in effect.

It is often desirable to provide the receiver with an erasure option that can be exercised when the received data are deemed unreliable. For fixed channels, Forney [3] derived the decision rule that provides the optimal tradeoff between the erasure and undetected-error probabilities, analogously to the Neyman-Pearson problem for binary hypothesis testing. Forney used the same framework to optimize the performance of list decoders; the probability of undetected errors is then replaced by the expected number of incorrect messages on the list. The size of the list is a random variable which equals 1 with high probability when communication is reliable. Bounds on the undetected-error and erasure exponents were subsequently derived by Telatar [4], [5] using a decoder parameterized by an asymmetric relation $\prec$ which is a function of the channel law.

For unknown channels, the problem of decoding with erasures was considered by Csiszár and Körner [1]. They derived attainable pairs of undetected-error and erasure exponents for any DMC. However, they did not indicate whether true universality is achievable, i.e., whether the exponents match Forney's exponents. Also they did not indicate whether their error exponents might be optimal in some weaker sense. The problem was recently revisited by Merhav and Feder [6], using a competitive minimax approach. The analysis of [6] yields lower bounds on a certain fraction of the optimal exponents. It is suggested in [6] that true universality might generally not be attainable, which would represent a fundamental difference with ordinary decoding.

The framework adopted for universality in this paper is based on the notion of compound DMCs, where the encoder and decoder know that the channel law belongs to a certain class $\mathcal{W}$ but do not know which channel law is in effect. This scenario has been widely used in the information theory and communications literature [1], [2], [7] and is particularly useful to model uncertainties about the DMC when a nominal channel model is available. For instance, the nominal model might be the binary-symmetric channel (BSC) with crossover probability $\rho=0.3$, and the compound class might be the set of all BSCs with crossover probability $0.2 \leq \rho \leq 0.4$.

This paper considers decoding with erasures for the compound DMC, with two goals in mind. The first is to construct a broad class of decision rules that can be optimized in an asymptotic Neyman-Pearson sense, analogously to universal hypothesis testing [8]-[10]. The second is to investigate the universality properties of the receiver, in particular conditions under which the exponents coincide with Forney's exponents. We first solve the problem of variable-size list decoders because it is 
simpler, and the solution to the ordinary problem of size-1 lists follows directly. We establish conditions under which our error exponents match Forney's exponents.

Following background material in Section II, the main results are given in Sections III-V. We also observe that in some problems the compound DMC approach is overly rigid and pessimistic. For such problems, we present in Section VI a simple and flexible extension of our method based on the relative minimax principle. In Section VII, we apply our results to a class of BSCs, which yields easily computable and insightful formulas. The paper concludes with a brief discussion in Section VIII. The proofs of the main results are given in the appendices.

\section{A. Notation}

We use uppercase letters for random variables, lowercase letters for individual values, and boldface fonts for sequences. The probability mass function (pmf) of a random variable $X \in \mathcal{X}$ is denoted by $p_{X}=\left\{p_{X}(x), x \in \mathcal{X}\right\}$, the probability of a set $\Omega$ under $p_{X}$ by $P_{X}(\Omega)$, and the expectation operator by $\mathbb{E}$. Entropy of a random variable $X$ is denoted by $H(X)$, and mutual information between two random variables $X$ and $Y$ is denoted by $I(X ; Y)=H(X)-H(X \mid Y)$, or by $I\left(p_{X Y}\right)$ when the dependency on $p_{X Y}$ should be explicit. The Kullback-Leibler divergence between two pmfs $p$ and $q$ is denoted by $D(p \| q)$. All logarithms are in base 2 . We denote by $f^{\prime}$ the derivative of a function $f$.

Denote by $p_{\boldsymbol{x}}$ the type of a sequence $\boldsymbol{x} \in \mathcal{X}^{N}$ ( $p_{\boldsymbol{x}}$ is an empirical pmf over $\mathcal{X}$ ) and by $T_{\boldsymbol{x}}$ the type class associated with $p_{\boldsymbol{x}}$, i.e., the set of all sequences of type $p_{\boldsymbol{x}}$. Likewise, denote by $p_{\boldsymbol{x} y}$ the joint type of a pair of sequences $(\boldsymbol{x}, \boldsymbol{y}) \in \mathcal{X}^{N} \times \mathcal{Y}^{N}$ (an empirical pmf over $\mathcal{X} \times \mathcal{Y}$ ) and by $T_{\boldsymbol{x} y}$ the type class associated with $p_{\boldsymbol{x} y}$, i.e., the set of all sequences of type $p_{\boldsymbol{x} \boldsymbol{y}}$. The conditional type $p_{\boldsymbol{y} \mid \boldsymbol{x}}$ of a pair of sequences $(\boldsymbol{x}, \boldsymbol{y})$ is defined as $p_{\boldsymbol{x} \boldsymbol{y}}(x, y) / p_{\boldsymbol{x}}(x)$ for all $x \in \mathcal{X}$ such that $p_{\boldsymbol{x}}(x)>0$. The conditional type class $T_{\boldsymbol{y} \mid \boldsymbol{x}}$ is the set of all sequences $\tilde{\boldsymbol{y}}$ such that $(\boldsymbol{x}, \tilde{\boldsymbol{y}}) \in T_{\boldsymbol{x} \boldsymbol{y}}$. We denote by $H(\boldsymbol{x})$ the entropy of the pmf $p_{\boldsymbol{x}}$ and by $H(\boldsymbol{y} \mid \boldsymbol{x})$ and $I(\boldsymbol{x} ; \boldsymbol{y})$ the conditional entropy and the mutual information for the joint pmf $p_{\boldsymbol{x} y}$, respectively. Recall that [1]

$$
\begin{aligned}
(N+1)^{-|\mathcal{X}|} 2^{N H(\boldsymbol{x})} & \leq\left|T_{\boldsymbol{x}}\right| \leq 2^{N H(\boldsymbol{x})} \\
(N+1)^{-|\mathcal{X}||\mathcal{Y}|} 2^{N H(\boldsymbol{y} \mid \boldsymbol{x})} & \leq\left|T_{\boldsymbol{y} \mid \boldsymbol{x}}\right| \leq 2^{N H(\boldsymbol{y} \mid \boldsymbol{x})} .
\end{aligned}
$$

We let $\mathcal{P}_{X}$ and $\mathcal{P}_{X}^{[N]}$ represent the set of all pmfs and empirical pmfs, respectively, for a random variable $X$. Likewise, $\mathcal{P}_{Y \mid X}$ and $\mathcal{P}_{Y \mid X}^{[N]}$ denote the set of all conditional pmfs and all empirical conditional pmfs, respectively, for a random variable $Y$ given $X$. The relation $f(N) \sim g(N)$ denotes asymptotic equality: $\lim _{N \rightarrow \infty} \frac{f(N)}{g(N)}=1$. The relations $f(N) \doteq g(N)$ and $f(N) \dot{\leq} g(N)$ denote equality and inequality on the exponential scale: $\lim _{N \rightarrow \infty} \frac{1}{N} \ln \frac{f(N)}{g(N)}=0$ and $\lim _{N \rightarrow \infty} \frac{1}{N} \ln \frac{f(N)}{g(N)} \leq 0$, respectively. We denote by $\mathbb{1}_{\{x \in \Omega\}}$ the indicator function of a set $\Omega$ and define $|t|^{+} \triangleq \max (0, t)$ and $\exp _{2}(t) \triangleq 2^{t}$. We adopt the notational convention that the minimum of a function over an empty set is $+\infty$.

The function-ordering notation $F \preceq G$ indicates that $F(t) \leq$ $G(t)$ for all $t$. Similarly, $F \succeq G$ indicates that $F(t) \geq G(t)$ for all $t$.

\section{DECODING With ERASURE AND List OPTIONS}

\section{A. Maximum-Likelihood Decoding}

In his 1968 paper [3], Forney studied the following erasure/list decoding problem. A length- $N$, rate- $R$ code $\mathcal{C}=$ $\{\boldsymbol{x}(m), m \in \mathcal{M}\}$ is selected, where $\mathcal{M}=\left\{1,2, \ldots, 2^{N R}\right\}$ is the message set and each codeword $\boldsymbol{x}(m) \in \mathcal{X}^{N}$. Upon selection of a message $m$, the corresponding $\boldsymbol{x}(m)$ is transmitted over a DMC $p_{Y \mid X}: \mathcal{X} \rightarrow \mathcal{Y}$. A set of decoding regions $\mathcal{D}_{m} \subseteq \mathcal{Y}^{N}, m \in \mathcal{M}$ is defined, and the decoder returns $\hat{m}=g(\boldsymbol{y})$ if and only if $\boldsymbol{y} \in \mathcal{D}_{m}$. For ordinary decoding, $\left\{\mathcal{D}_{m}, m \in \mathcal{M}\right\}$ form a partition of $\mathcal{Y}^{N}$. When an erasure option is introduced, the decision space is extended to $\mathcal{M} \cup \emptyset$, where $\emptyset$ denotes the erasure symbol. The erasure region $\mathcal{D}_{\emptyset}$ is the complement of $\cup_{m \in \mathcal{M}} \mathcal{D}_{m}$ in $\mathcal{Y}^{N}$. An undetected error arises if $m$ was transmitted but $\boldsymbol{y}$ lies in the decoding region of some other message $i \neq m$. This event is given by

$$
\mathcal{E}_{i}=\left\{(m, \boldsymbol{y}): \boldsymbol{y} \in \underset{i \in \mathcal{M} \backslash\{m\}}{\bigcup} \mathcal{D}_{i}\right\}
$$

where the subscript $i$ stands for "incorrect message." Hence

$$
\begin{aligned}
\operatorname{Pr}\left[\mathcal{E}_{i}\right] & =\frac{1}{|\mathcal{M}|} \sum_{m \in \mathcal{M}} \sum_{\boldsymbol{y} \in \bigcup_{i \in \mathcal{M} \backslash\{m\}} p_{i}} p_{Y \mid X}^{N}(\boldsymbol{y} \mid \boldsymbol{x}(m)) \\
& =\frac{1}{|\mathcal{M}|} \sum_{m \in \mathcal{M}} \sum_{i \in \mathcal{M} \backslash\{m\}} \sum_{\boldsymbol{y} \in \mathcal{D}_{i}} p_{Y \mid X}^{N}(\boldsymbol{y} \mid \boldsymbol{x}(m))
\end{aligned}
$$

where the second equality holds because the decoding regions are disjoint.

The erasure event is given by

$$
\mathcal{E}_{\emptyset}=\left\{(m, \boldsymbol{y}): \boldsymbol{y} \in \mathcal{D}_{\emptyset}\right\}
$$

and has probability

$$
\operatorname{Pr}\left[\mathcal{E}_{\emptyset}\right]=\frac{1}{|\mathcal{M}|} \sum_{m \in \mathcal{M}} \sum_{\boldsymbol{y} \in \mathcal{D}_{\emptyset}} p_{Y \mid X}^{N}(\boldsymbol{y} \mid \boldsymbol{x}(m)) .
$$

The total error event is given by $\mathcal{E}_{\text {err }}=\mathcal{E}_{i} \cup \mathcal{E}_{\emptyset}$. The decoder is generally designed so that $\operatorname{Pr}\left[\mathcal{E}_{i}\right] \ll \operatorname{Pr}\left[\mathcal{E}_{\emptyset}\right]$, so $\operatorname{Pr}\left[\mathcal{E}_{\text {err }}\right] \approx$ $\operatorname{Pr}\left[\mathcal{E}_{\emptyset}\right]$.

Analogously to the Neyman-Pearson problem, one wishes to design the decoding regions to obtain an optimal tradeoff between $\operatorname{Pr}\left[\mathcal{E}_{i}\right]$ and $\operatorname{Pr}\left[\mathcal{E}_{\emptyset}\right]$. Forney proved that the class of decision rules, given in (2.4) at the bottom of the page, is optimal, where $T \geq 0$ is a free parameter trading off $\operatorname{Pr}\left[\mathcal{E}_{i}\right]$ against $\operatorname{Pr}\left[\mathcal{E}_{\emptyset}\right]$. The nonnegativity constraint on $T$ ensures that $\hat{m}$ is uniquely defined for any given $\boldsymbol{y}$. There is no other decision rule that yields simultaneously a lower value for $\operatorname{Pr}\left[\mathcal{E}_{i}\right]$ and for $\operatorname{Pr}\left[\mathcal{E}_{\emptyset}\right]$.

$$
g_{M L}(\boldsymbol{y})=\left\{\begin{array}{lll}
\hat{m} & : & \text { if } p_{Y \mid X}^{N}(\boldsymbol{y} \mid \boldsymbol{x}(\hat{m}))>e^{N T} \sum_{i \neq \hat{m}} p_{Y \mid X}^{N}(\boldsymbol{y} \mid \boldsymbol{x}(i)) \\
\emptyset & : & \text { else }
\end{array}\right.
$$


A conceptually simple (but suboptimal) alternative to (2.4) is (2.5) shown at the bottom of the page, where the decision is made based on the two highest likelihood scores.

If one chooses $T<0$, there is generally more than one value of $\hat{m}$ that satisfies (2.4), and $g_{M L}$ may be viewed as a list decoder that returns the list of all such $\hat{m}$. Denote by $N_{i}$ the number of incorrect messages on the list. Since the decoding regions $\left\{\mathcal{D}_{m}, m \in \mathcal{M}\right\}$ overlap, the average number of incorrect messages in the list

$$
\mathbb{E}\left[N_{i}\right]=\frac{1}{|\mathcal{M}|} \sum_{m \in \mathcal{M}} \sum_{i \in \mathcal{M} \backslash\{m\}} \sum_{\boldsymbol{y} \in \mathcal{D}_{i}} p_{Y \mid X}^{N}(\boldsymbol{y} \mid \boldsymbol{x}(m)),
$$

no longer coincides with $\operatorname{Pr}\left[\mathcal{E}_{i}\right]$ in (2.2).

For the rule (2.4) applied to symmetric channels, Forney showed that the following error exponents are achievable for all $\Delta$ such that $R^{\text {conj }} \leq R+\Delta \leq C$ :

$$
\begin{aligned}
& E_{i}(R, \Delta)=E_{\mathrm{sp}}(R)+\Delta \\
& E_{\emptyset}(R, \Delta)=E_{\mathrm{sp}}(R+\Delta)
\end{aligned}
$$

where $E_{\mathrm{sp}}(R)$ is the sphere-packing exponent, and $R^{\text {conj }}$ is the conjugate rate, defined as the rate for which the slope of $E_{\mathrm{sp}}(\cdot)$ is the reciprocal of the slope at rate $R$

$$
E_{\mathrm{sp}}^{\prime}\left(R^{\mathrm{conj}}\right)=\frac{1}{E_{\mathrm{sp}}^{\prime}(R)} .
$$

The exponents of (2.7) are achieved using independent and identically distributed (i.i.d.) codes.

\section{B. Universal Decoding}

When the channel law $p_{Y \mid X}$ is unknown, maximum-likelihood decoding cannot be used. For constant-composition codes with type $p_{X}$, the MMI decoder takes the form

$$
g_{\mathrm{MMI}}(\boldsymbol{y})=\arg \max _{i \in \mathcal{M}} I(\boldsymbol{x}(i) ; \boldsymbol{y}) .
$$

Csiszár and Körner [1, pp. 174-178] extended the MMI decoder to include an erasure option, using the decision rule (2.10) also shown at the bottom of the page, where $\Delta \geq 0$ and $\lambda>1$.
They derived the following error exponents for the resulting undetected-error and erasure events:

$$
\left\{E_{r, \lambda}\left(R, p_{X}, p_{Y \mid X}\right)+\Delta, E_{r, 1 / \lambda}\left(R+\Delta, p_{X}, p_{Y \mid X}\right)\right\},
$$

$\forall p_{Y \mid X}$

where

$$
\begin{aligned}
& E_{r, \lambda}\left(R, p_{X}, p_{Y \mid X}\right) \\
& \quad=\min _{\tilde{p}_{Y \mid X}}\left\{D\left(\tilde{p}_{Y \mid X} \| p_{Y \mid X} \mid p_{X}\right)+\lambda\left|I\left(p_{X}, \tilde{p}_{Y \mid X}\right)-R\right|^{+}\right\} .
\end{aligned}
$$

While $\Delta$ and $\lambda$ are tradeoff parameters, they did not mention whether the decision rule (2.10) satisfies any Neyman-Pearson type optimality criterion.

A different approach was recently proposed by Merhav and Feder [6]. They raised the possibility that the achievable pairs of undetected-error and erasure exponents might be smaller than in the known-channel case and proposed a decision rule based on the competitive minimax principle. This rule is parameterized by a scalar parameter $0 \leq \xi \leq 1$ which represents a fraction of the optimal exponents (for the known-channel case) that their decoding procedure is guaranteed to achieve. Decoding involves explicit maximization of a cost function over the compound DMC family, analogously to a generalized likelihood ratio test (GLRT). The rule coincides with the GLRT when $\xi=0$, but the choice of $\xi$ can be optimized. They conjectured that the highest achievable $\xi$ is lower than 1 in general, and derived a computable lower bound on that value.

\section{III. $\mathcal{F}$-MMI CLASS OF DECODERS}

\section{A. Decoding Rule}

Assume that random constant-composition codes with type $p_{X}$ are used, and that the DMC $p_{Y \mid X}$ belongs to a compact subset $\mathcal{W}$ of $\mathcal{P}_{Y \mid X}$. The decoder knows $\mathcal{W}$ but not which $p_{Y \mid X}$ is in effect.

Analogously to (2.10), our proposed decoding rule is a test based on the empirical mutual informations for each candidate message and its highest scoring competitor. Let $\mathcal{F}$ be the class of continuous, nondecreasing functions $F:\left[-R, H\left(p_{X}\right)-R\right] \rightarrow$ $\mathbb{R}$. The decision rule indexed by $F \in \mathcal{F}$ takes the form of (3.1) shown at the bottom of the page. Given a candidate message $\hat{m}$,

$$
g_{M L, 2}(\boldsymbol{y})=\left\{\begin{array}{lll}
\hat{m} & : & \text { if } p_{Y \mid X}^{N}(\boldsymbol{y} \mid \boldsymbol{x}(\hat{m}))>e^{N T} \max _{i \neq \hat{m}} p_{Y \mid X}^{N}(\boldsymbol{y} \mid \boldsymbol{x}(i)) \\
\emptyset & : & \text { else }
\end{array}\right.
$$

$$
g_{\lambda, \Delta}(\boldsymbol{y})=\left\{\begin{array}{lll}
\hat{m} & : & \text { if } I(\boldsymbol{x}(\hat{m}) ; \boldsymbol{y})>R+\Delta+\lambda \max _{i \neq \hat{m}}|I(\boldsymbol{x}(i) ; \boldsymbol{y})-R|^{+} \\
\emptyset & : & \text { else }
\end{array}\right.
$$

$$
g_{F}(\boldsymbol{y})=\left\{\begin{array}{lll}
\hat{m} & : & \text { if } I(\boldsymbol{x}(\hat{m}) ; \boldsymbol{y})>R+\max _{i \neq \hat{m}} F(I(\boldsymbol{x}(i) ; \boldsymbol{y})-R) \\
\emptyset & : & \text { else. }
\end{array}\right.
$$


the function $F$ weighs the score of the best competing codeword. The decoder outputs $\hat{m}$ if its empirical mutual information score is large enough relative to its strongest competitor, as weighted by $F .{ }^{1}$ Since $0 \leq I(\boldsymbol{x}(i) ; \boldsymbol{y}) \leq H\left(p_{X}\right)$, all values of $F(t)$ for $t \notin\left[-R, H\left(p_{X}\right)-R\right]$ are equivalent in terms of the decision rule (3.1).

The choice $F(t)=t$ results in the MMI decoding rule (2.9), and

$$
F(t)=\Delta+\lambda|t|^{+}
$$

(two-parameter family of functions) results in the Csiszár-Körner rule (2.10).

One may further require that $F(t) \geq t$ to guarantee that $\hat{m}=\arg \max _{i} I(\boldsymbol{x}(i) ; \boldsymbol{y})$, as can be verified by direct substitution into (3.1). In this case, the decision is whether the decoder should output the highest scoring message or output an erasure decision.

When the restriction $F(t) \geq t$ is not imposed, the decision rule (3.1) is ambiguous because more than one $\hat{m}$ could satisfy the inequality in (3.1). Then (3.1) may be viewed as a list decoder that returns the list of all such $\hat{m}$, similarly to (2.4).

The Csiszár-Körner decision rule parameterized by $F$ in (3.2) is nonambiguous for $\lambda \geq 1$. Note that there is an error in Theorem 5.11 and Corollary 5.11A of [1, p. 175], where the condition $\lambda>0$ should be replaced with $\lambda \geq 1[11]$.

In the limit as $\lambda \downarrow 0,(3.2)$ leads to the simple decoder that lists all messages whose empirical mutual information score exceeds $R+\Delta$. If a list decoder is not desired, a simple variation on (3.2) when $0<\lambda<1$ is

$$
F(t)=\left\{\begin{array}{lll}
\Delta+\lambda|t|^{+} & : & t \leq \frac{\Delta}{1-\lambda} \\
t & : & \text { else. }
\end{array}\right.
$$

It is also worth noting that the function $F(t)=\Delta+t$ may be thought of as an empirical version of Forney's suboptimal decoding rule $(2.5)$, with $T=\Delta$. Indeed, using the identity $I(\boldsymbol{x} ; \boldsymbol{y})=H(\boldsymbol{y})-H(\boldsymbol{y} \mid \boldsymbol{x})$ and viewing the negative empirical equivocation

$$
-H(\boldsymbol{y} \mid \boldsymbol{x})=\sum_{x, y} p_{\boldsymbol{x} \boldsymbol{y}}(x, y) \ln p_{\boldsymbol{y} \mid \boldsymbol{x}}(y \mid x)
$$

as an empirical version of the normalized loglikelihood

$$
\frac{1}{N} \ln p_{Y \mid X}^{N}(\boldsymbol{y} \mid \boldsymbol{x})=\sum_{x, y} p_{\boldsymbol{x} \boldsymbol{y}}(x, y) \ln p_{Y \mid X}(y \mid x),
$$

${ }^{1}$ Note that the function $F$ could have been defined slightly differently as $G(t)=R+F(t-R)$. The right side of (3.1) would be written more compactly as $\max _{i \neq \hat{m}} G(I(\boldsymbol{x}(i) ; \boldsymbol{y}))$ but the resulting expressions (4.3) and (4.7) for random-coding exponents would be somewhat less elegant. we may rewrite (2.5) and (3.1) respectively as(3.3) at the bottom of the page, and

$$
g_{F}(\boldsymbol{y})= \begin{cases}\hat{m} & : \text { if }-H(\boldsymbol{y} \mid \boldsymbol{x}(\hat{m}))>\Delta+\max _{i \neq \hat{m}}[-H(\boldsymbol{y} \mid \boldsymbol{x}(i))] \\ \emptyset \quad: \text { else. }\end{cases}
$$

While this observation does not imply $F(t)=\Delta+t$ is an optimal choice for $F$, one might intuitively expect optimality in some regime.

\section{B. Error Exponents}

For a random-coding strategy using constant-composition codes with type $p_{X}$, the expected number of incorrect messages on the list $\mathbb{E}\left[N_{i}\right]$ and the erasure probability, $\operatorname{Pr}\left[\mathcal{E}_{\emptyset}\right]$ may be viewed as functions of $R, p_{X}, p_{Y \mid X}$, and $F$.

Definition 3.1: A pair

$$
\left\{E_{i}\left(R, p_{X}, p_{Y \mid X}, F\right), E_{\emptyset}\left(R, p_{X}, p_{Y \mid X}, F\right)\right\}
$$

of incorrect-message and erasure exponents is said to be universally attainable over $\mathcal{W}$ for random codes of rate $R$ and type $p_{X}$ if the expected number of incorrect messages on the list and the erasure probability satisfy

$$
\begin{array}{r}
\mathbb{E}\left[N_{i}\right] \leq \exp _{2}\left\{-N\left[E_{i}\left(R, p_{X}, p_{Y \mid X}, F\right)-\epsilon\right]\right\} \\
\operatorname{Pr}\left[\mathcal{E}_{\emptyset}\right] \leq \exp _{2}\left\{-N\left[E_{\emptyset}\left(R, p_{X}, p_{Y \mid X}, F\right)-\epsilon\right]\right\}, \\
\forall p_{Y \mid X} \in \mathcal{W}
\end{array}
$$

for any $\epsilon>0$ and $N$ greater than some $N_{0}(\epsilon)$.

The worst case exponents (over all $p_{Y \mid X} \in \mathcal{W}$ ) are denoted by

$$
\begin{aligned}
& E_{i}\left(R, p_{X}, \mathcal{W}, F\right) \triangleq \min _{p_{Y \mid X} \in \mathcal{W}} E_{i}\left(R, p_{X}, p_{Y \mid X}, F\right) \\
& E_{\emptyset}\left(R, p_{X}, \mathcal{W}, F\right) \triangleq \min _{p_{Y \mid X} \in \mathcal{W}} E_{\emptyset}\left(R, p_{X}, p_{Y \mid X}, F\right) .
\end{aligned}
$$

Our problem is to maximize the erasure exponent $E_{\emptyset}\left(R, p_{X}, \mathcal{W}, F\right)$ subject to the constraint that the incorrect-message exponent $E_{i}\left(R, p_{X}, \mathcal{W}, F\right)$ is at least equal to some prescribed value $\alpha$. This is an asymptotic Neyman-Pearson problem. We shall focus on the regime of practical interest where erasures are more acceptable than undetected errors

$$
E_{\emptyset}\left(R, p_{X}, \mathcal{W}, F\right) \leq E_{i}\left(R, p_{X}, \mathcal{W}, F\right) .
$$

We emphasize that asymptotic Neyman-Pearson optimality of the decision rule holds only in a restricted sense, namely, with respect to the $\mathcal{F}$-MMI class (3.1).

$$
g_{M L, 2}(\boldsymbol{y})=\left\{\begin{array}{lll}
\hat{m} & : & \text { if } \frac{1}{N} \ln p_{Y \mid X}^{N}(\boldsymbol{y} \mid \boldsymbol{x}(\hat{m}))>T+\max _{i \neq \hat{m}} \frac{1}{N} \ln p_{Y \mid X}^{N}(\boldsymbol{y} \mid \boldsymbol{x}(i)) \\
\emptyset & : \text { else }
\end{array}\right.
$$


Specifically, given $R$ and $\mathcal{W}$, we seek the solution to the constrained optimization problem

$$
\begin{aligned}
& E_{\emptyset}^{*}(R, \mathcal{W}, \alpha) \\
& \triangleq \max _{p_{X}} \max _{F \in \mathcal{F}\left(R, p_{X}, \mathcal{W}, \alpha\right)} \min _{p_{Y \mid X} \in \mathcal{W}} E_{\emptyset}\left(R, p_{X}, p_{Y \mid X}, F\right)
\end{aligned}
$$

where $\mathcal{F}\left(R, p_{X}, \mathcal{W}, \alpha\right)$ is the set of functions $F$ that satisfy

$$
\min _{p_{Y \mid X} \in \mathcal{W}} E_{i}\left(R, p_{X}, p_{Y \mid X}, F\right) \geq \alpha
$$

as well as the continuity and monotonicity conditions mentioned above (3.1).

If we were able to choose $F$ as a function of $p_{Y \mid X}$, we would do at least as well as in (3.9) and achieve the erasure exponent

$$
\begin{aligned}
E_{\emptyset}^{* *}(R, \mathcal{W}, \alpha) \triangleq & \max _{p_{X}} \min _{p_{Y \mid X} \in \mathcal{W}} \max _{F \in \mathcal{F}\left(R, p_{X}, p_{Y \mid X}, \alpha\right)} \\
& E_{\emptyset}\left(R, p_{X}, p_{Y \mid X}, F\right) \\
\geq & E_{\emptyset}^{*}(R, \mathcal{W}, \alpha) .
\end{aligned}
$$

The max min max order for this optimization corresponds to a widely used communications scenario without feedback where each player only knows the strategy of the previous player(s) and so the encoder does not know the channel law, but the decoder does. We shall be particularly interested in characterizing $(R, \mathcal{W}, \alpha)$ for which the decoder incurs no penalty for not knowing $p_{Y \mid X}$, i.e.,

- equality holds in (3.11), and

- the optimal exponents $E_{i}\left(R, p_{X}, p_{Y \mid X}, F\right)$ and $E_{\emptyset}\left(R, p_{X}, p_{Y \mid X}, F\right)$ in (3.10) and (3.9) coincide with Forney's exponents in (2.7) for all $p_{Y \mid X} \in \mathcal{W}$, the second property being stronger than the first.

\section{Basic Properties of $F$}

To simplify the derivations, it is convenient to slightly strengthen the requirement that $F$ be nondecreasing, and work with (strictly) increasing functions $F$ instead. Then the maxima over $F$ in (3.9) and (3.11) are replaced with suprema, but of course their value remains the same.

To each monotonically increasing function $F \in \mathcal{F}$ corresponds an inverse $F^{-1}$, such that

$$
F(t)=u \Leftrightarrow F^{-1}(u)=t .
$$

Since $F$ is continuous and monotonically increasing, elementary properties satisfied by $F^{-1}$ include:

(P1) $F^{-1}$ is continuous and increasing over its range.

(P2) If $F \preceq G$, then $F^{-1} \succeq G^{-1}$.

(P3) $G(t)=F(t)+\Delta \Leftrightarrow G^{-1}(t)=F^{-1}(t-\Delta)$.

(P4) If $F$ is differentiable, then $\frac{d F(t)}{d t}=1 / \frac{d F^{-1}(t)}{d t}$.

(P5) If $F$ is convex, then $F^{-1}$ is concave.

(P6) The domain of $F^{-1}$ is the range of $F$, and vice versa.

The function $F^{-1}$ that appears in the statement of our main results can still be defined if $F$ is not strictly increasing, but some care must be taken, in particular in the definition of the domain of $F^{-1}$. For instance, given parameters $\lambda, \epsilon>0$, the function

$$
F(t)=\left\{\begin{array}{lll}
\Delta+\lambda t & : & 0 \leq t \leq H\left(p_{X}\right)-R \\
\Delta+\epsilon t & : & -R \leq t \leq 0
\end{array}\right.
$$

is increasing and converges in the sup norm to (3.2) as $\epsilon \downarrow 0$. The inverse of (3.12) is given by

$$
F^{-1}(t)= \begin{cases}\lambda^{-1}(t-\Delta) & : \quad 0 \leq t-\Delta \leq \lambda\left[H\left(p_{X}\right)-R\right] \\ \epsilon^{-1}(t-\Delta) & : \quad-\epsilon R \leq t-\Delta \leq 0 .\end{cases}
$$

In the limit case $\epsilon=0$, the domain of $F^{-1}$ reduces to $[\Delta, \Delta+$ $\left.\lambda\left(H\left(p_{X}\right)-R\right)\right]$, and ${ }^{2}$

$$
F^{-1}(t)=\frac{t-\Delta}{\lambda}, \text { for } t \geq \Delta .
$$

In the limit case $\lambda=\epsilon=0$, corresponding to $F(t) \equiv \Delta$, the domain of $F^{-1}$ reduces to the singleton $\{\Delta\}$.

\section{RANDOM-CODING AND SPHERE-PACKING EXPONENTS}

The sphere-packing exponent for channel $p_{Y \mid X}$ is defined as $E_{\mathrm{sp}}\left(R, p_{X}, p_{Y \mid X}\right)$

$$
\triangleq \min _{\tilde{p}_{Y \mid X}: I\left(p_{X}, \tilde{p}_{Y \mid X}\right) \leq R} D\left(\tilde{p}_{Y \mid X} \| p_{Y \mid X} \mid p_{X}\right)
$$

and as $\infty$ if the minimization above is over an empty set. The function $E_{\mathrm{sp}}\left(R, p_{X}, p_{Y \mid X}\right)$ is convex, nonincreasing, and continuous in $p_{Y \mid X}$. The first two properties are given in [1, Lemma 5.4]. The third property holds because the minimand of (4.1) may be viewed as a functional $\Phi\left(\tilde{p}_{Y \mid X}, p_{Y \mid X}\right)$, and the left side of (4.1) as a functional $E\left(p_{Y \mid X}\right)=\min _{\tilde{p}_{Y \mid X}} \Phi\left(\tilde{p}_{Y \mid X}, p_{Y \mid X}\right)$. Since $\Phi$ is continuous in $\left(\tilde{p}_{Y \mid X}, p_{Y \mid X}\right)$ with respect to $L^{1}$ norm, (4.1) is also a continuous functional of $p_{Y \mid X}$.

The sphere-packing exponent for class $\mathcal{W}$ is defined as

$$
E_{\mathrm{sp}}\left(R, p_{X}, \mathcal{W}\right) \triangleq \min _{p_{Y \mid X} \in \mathcal{W}} E_{\mathrm{sp}}\left(R, p_{X}, p_{Y \mid X}\right)
$$

The function $E_{\mathrm{sp}}\left(R, p_{X}, \mathcal{W}\right)$ is continuous and nonincreasing in $R$. In some cases, $E_{\mathrm{sp}}\left(R, p_{X}, \mathcal{W}\right)$ is also convex and continuously differentiable in $R$, e.g., when the same $p_{Y \mid X}$ achieves the minimum in (4.2) at all rates. Denote by $R_{\infty}\left(p_{X}, \mathcal{W}\right)$ the infimum of the rates $R$ such that $E_{\mathrm{sp}}\left(R, p_{X}, \mathcal{W}\right)<\infty$, and by

$$
I\left(p_{X}, \mathcal{W}\right) \triangleq \min _{p_{Y \mid X} \in \mathcal{W}} I\left(p_{X}, p_{Y \mid X}\right)
$$

the supremum of $R$ such that $E_{\mathrm{sp}}\left(R, p_{X}, \mathcal{W}\right)>0$.

The modified random coding exponent for channel $p_{Y \mid X}$ and for class $\mathcal{W}$ are, respectively, defined as

$$
\begin{aligned}
E_{r, F}\left(R, p_{X}, p_{Y \mid X}\right) \triangleq \min _{\tilde{p}_{Y \mid X}}[ & D\left(\tilde{p}_{Y \mid X} \| p_{Y \mid X} \mid p_{X}\right) \\
& \left.+F\left(I\left(p_{X}, \tilde{p}_{Y \mid X}\right)-R\right)\right]
\end{aligned}
$$

\footnotetext{
${ }^{2}$ Any nondecreasing $F$ can be written as the limit of a sequence of increasing functions converging in the sup norm. Since $F$ is defined over a finite interval, this implies convergence in the $L^{1}$ norm. The sequence of inverse functions converges in the $L^{1}$ norm to a right-continuous function which may be defined as $F^{-1}$. Since $E_{r, F}$ defined in (4.3) is continuous with respect to $F$ in the $L^{1}$ norm, it follows that Propositions 4.1 and 4.2 hold for nondecreasing $F$ and not just for increasing $F$.
} 


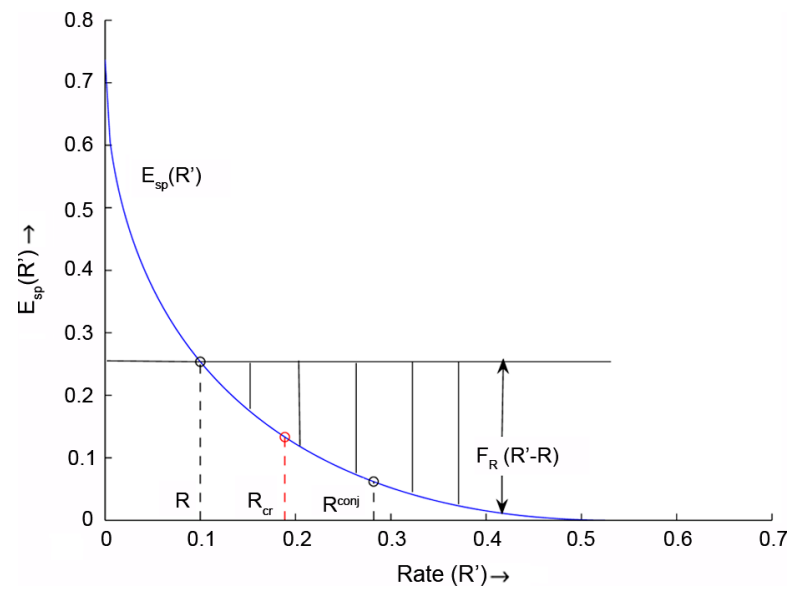

Fig. 1. A graphical depiction of the function $F_{R, p_{X}, \mathcal{W}}\left(R^{\prime}-R\right)$ versus $R^{\prime}$ when $R=0.1, \mathcal{W}$ is the family of BSCs with crossover probability $\rho \leq 0.1$ (capacity $C(\rho) \geq 0.53$ ), and $p_{X}$ is the uniform pmf over $\{0,1\}$. The function is evaluated from the sphere-packing exponent $E_{\mathrm{sp}}\left(R^{\prime}\right)$ for class $\mathcal{W}$.

and

$$
E_{r, F}\left(R, p_{X}, \mathcal{W}\right) \triangleq \min _{p_{Y \mid X} \in \mathcal{W}} E_{r, F}\left(R, p_{X}, p_{Y \mid X}\right)
$$

We may view the minimand of (4.3) as a functional $\Phi\left(F, \tilde{p}_{Y \mid X}\right)$ and the left side of (4.3) as a functional

$$
E(F)=\min _{p_{Y \mid X}} \Phi\left(F, \tilde{p}_{Y \mid X}\right)
$$

Since $\Phi$ is continuous in $\left(F, \tilde{p}_{Y \mid X}\right),(4.3)$ is a continuous functional of $F$. When $F(t)=|t|^{+},(4.3)$ is just the usual random coding exponent.

Define the function

$$
F_{R, p_{X}, \mathcal{W}}(t) \triangleq E_{\mathrm{sp}}\left(R, p_{X}, \mathcal{W}\right)-E_{\mathrm{sp}}\left(R+t, p_{X}, \mathcal{W}\right)
$$

which is depicted in Fig. 1 for a BSC example to be analyzed in Section VII. This function is increasing for $R_{\infty}\left(p_{X}, \mathcal{W}\right)-$ $R \leq t \leq I\left(p_{X}, \mathcal{W}\right)-R$ and satisfies the following elementary properties:

$$
\begin{aligned}
F_{R, p_{X}, \mathcal{W}}(0) & =0 \\
F_{R, p_{X}, \mathcal{W}}^{\prime}(t) & =-E_{\mathrm{sp}}^{\prime}\left(R+t, p_{X}, \mathcal{W}\right)
\end{aligned}
$$$$
E_{\mathrm{sp}}\left(R^{\prime}, p_{X}, \mathcal{W}\right)+F_{R, p_{X}, \mathcal{W}}\left(R^{\prime}-R\right) \equiv E_{\mathrm{sp}}\left(R, p_{X}, \mathcal{W}\right) \text {. }
$$

If $E_{\mathrm{sp}}\left(R, p_{X}, \mathcal{W}\right)$ is convex in $R$, then $F_{R, p_{X}, \mathcal{W}}(t)$ is concave in $t$.

Proposition 4.1: The modified random coding exponent $E_{r, F}\left(R, p_{X}, p_{Y \mid X}\right)$ satisfies the following properties.

(i) $E_{r, F}\left(R, p_{X}, p_{Y \mid X}\right)$ is decreasing in $R$ for all $R \leq$ $I\left(p_{X}, p_{Y \mid X}\right)$.

(ii) If $F \preceq G$, then

$$
E_{r, F}\left(R, p_{X}, p_{Y \mid X}\right) \leq E_{r, G}\left(R, p_{X}, p_{Y \mid X}\right) .
$$

(iii) $E_{r, F}\left(R, p_{X}, p_{Y \mid X}\right)$ is related to the sphere packing exponent as follows:

$$
\begin{aligned}
& E_{r, F}\left(R, p_{X}, p_{Y \mid X}\right) \\
& \quad=\min _{R^{\prime}}\left[E_{\mathrm{sp}}\left(R^{\prime}, p_{X}, p_{Y \mid X}\right)+F\left(R^{\prime}-R\right)\right] .
\end{aligned}
$$

(iv) The preceding properties hold with $\mathcal{W}$ in place of $p_{Y \mid X}$ in the arguments of the functions $E_{r, F}, E_{\mathrm{sp}}$, and $I$.

The proof of these properties is given in Appendix A. Part (iii) is a variation on Lemma 5.4 and its corollary in [1, p. 168]. Also note that while $E_{r, F}\left(R, p_{X}, p_{Y \mid X}\right)$ is convex in $R$ for some choices of $F$, including (3.2), that property does not extend to arbitrary $F$.

Proposition 4.2: The following pair of incorrect-message and erasure exponents are universally attainable over $\mathcal{W}$ (recall Definition 3.1) under the decision rule (3.1):

$$
\begin{aligned}
& E_{i}\left(R, p_{X}, p_{Y \mid X}, F\right)=E_{r, F}\left(R, p_{X}, p_{Y \mid X}\right) \\
& E_{\emptyset}\left(R, p_{X}, p_{Y \mid X}, F\right)=E_{r,\left|F^{-1}\right|^{+}}\left(R, p_{X}, p_{Y \mid X}\right), \quad \forall F \in \mathcal{F} .
\end{aligned}
$$

Proof: See Appendix B.

If $F(t)=|t|^{+}$, then $F^{-1}(t)=t$ for $t \geq 0$, and both (4.8) and (4.9) reduce to the ordinary random-coding exponent.

If the channel is not reliable enough in the sense that $I\left(p_{X}, p_{Y \mid X}\right)<R+F(0)$, then

$$
F^{-1}\left(I\left(p_{X}, p_{Y \mid X}\right)-R\right) \leq F^{-1}(F(0))=0
$$

and from (4.9) we obtain $E_{\emptyset}\left(R, p_{X}, p_{Y \mid X}, F\right)=0$ because the minimizing $\tilde{p}_{Y \mid X}$ in the expression for $E_{r,\left|F^{-1}\right|^{+}}$is equal to $p_{Y \mid X}$. Checking (3.1), a heuristic interpretation for the zero erasure exponent is that $I(\boldsymbol{x}(m) ; \boldsymbol{y}) \approx I\left(p_{X}, p_{Y \mid X}\right)$ with high probability when $m$ is the transmitted message, and $\max _{i \neq m} I(\boldsymbol{x}(m) ; \boldsymbol{y}) \approx R$ (obtained using (B.4) with $\nu=R$ and the union bound). Hence $m$ fails the test (3.1) with high probability.

\section{W-Optimal ChOice of $F$}

In this section, we view the incorrect-message and erasure exponents as functionals of $|F|^{+}$and examine optimal tradeoffs between them. It is instructive to first consider the one-parameter family

$$
F(t) \equiv \Delta \geq 0
$$

which corresponds to a thresholding rule in (3.1). Using (4.7)-(4.9), we obtain

$$
\begin{aligned}
& E_{i}\left(R, p_{X}, p_{Y \mid X}, F\right)=E_{r, F}\left(R, p_{X}, p_{Y \mid X}\right) \equiv \Delta \\
& E_{\emptyset}\left(R, p_{X}, p_{Y \mid X}, F\right) \equiv E_{\mathrm{sp}}\left(R+\Delta, p_{Y \mid X}\right)
\end{aligned}
$$

where (5.2) holds because the minimum in (4.7) is achieved at $R^{\prime}=I\left(p_{X}, p_{Y \mid X}\right)$, in which case the sphere-packing exponent is 0 ; and (5.3) is obtained by viewing $F(t) \equiv \Delta$ as the limit of a sequence of increasing functions, as described at the end of Section III-C. Two extreme choices for $\Delta$ are 0 and $I\left(p_{X}, \mathcal{W}\right)-$ $R$ because in each case one error exponent is zero and the other one is positive. One would expect that better tradeoffs can be achieved using a broader class of functions $F$, though.

Recalling (3.9), (3.10), and using (4.8) and (4.9), we seek the solution to the following two asymptotic Neyman-Pearson optimization problems. For list decoding, find

$$
E_{\emptyset}^{L}(R, \mathcal{W}, \alpha)=\max _{p_{X}} E_{\emptyset}^{L}\left(R, p_{X}, \mathcal{W}, \alpha\right)
$$


where the cost function

$$
\begin{aligned}
E_{\emptyset}^{L}\left(R, p_{X}, \mathcal{W}, \alpha\right) & \sup _{F \in \mathcal{F}^{L}\left(R, p_{X}, \mathcal{W}, \alpha\right)} E_{r,\left|F^{-1}\right|^{+}}\left(R, p_{X}, \mathcal{W}\right)
\end{aligned}
$$

and the feasible set $\mathcal{F}^{L}\left(R, p_{X}, \mathcal{W}, \alpha\right)$ is the set of continuous, increasing functions $F$ that yield an incorrect-message exponent at least equal to $\alpha$

$$
E_{i}\left(R, p_{X}, \mathcal{W}, F\right)=E_{r, F}\left(R, p_{X}, \mathcal{W}\right) \geq \alpha .
$$

For classical decoding (list size $\leq 1$ ), find

$$
E_{\emptyset}(R, \mathcal{W}, \alpha)=\max _{p_{X}} E_{\emptyset}\left(R, p_{X}, \mathcal{W}, \alpha\right)
$$

where

$$
\begin{aligned}
E_{\emptyset}\left(R, p_{X}, \mathcal{W}, \alpha\right) & \sup _{F \in \mathcal{F}\left(R, p_{X}, \mathcal{W}, \alpha\right)} E_{r,\left|F^{-1}\right|^{+}}\left(R, p_{X}, \mathcal{W}\right)
\end{aligned}
$$

and $\mathcal{F}\left(R, p_{X}, \mathcal{W}, \alpha\right)$ is the subset of functions in $\mathcal{F}^{L}\left(R, p_{X}, \mathcal{W}, \alpha\right)$ that satisfy $F(t) \geq t$, i.e., the decoder outputs at most one message.

Since $\mathcal{F}\left(R, p_{X}, \mathcal{W}, \alpha\right) \subset \mathcal{F}^{L}\left(R, p_{X}, \mathcal{W}, \alpha\right)$, we have

$$
E_{\emptyset}\left(R, p_{X}, \mathcal{W}, \alpha\right) \leq E_{\emptyset}^{L}\left(R, p_{X}, \mathcal{W}, \alpha\right) .
$$

The remainder of this paper focuses on the class of variable-size list decoders associated with $\mathcal{F}^{L}$ because the error exponent tradeoffs are at least as good as those associated with $\mathcal{F}$, and the corresponding error exponents take a more concise form.

Define the critical rate $R_{c r}\left(p_{X}, \mathcal{W}\right)$ as the rate at which the derivative $E_{\mathrm{sp}}^{\prime}\left(\cdot, p_{X}, \mathcal{W}\right)=-1$ (assuming this derivative exists), and

$$
\Delta \triangleq \alpha-E_{\mathrm{sp}}\left(R, p_{X}, \mathcal{W}\right) .
$$

Analogously to (2.8), two rates $R_{1}$ and $R_{2}$ are said to be conjugate given $p_{X}$ and $\mathcal{W}$ if the corresponding slopes of $E_{\mathrm{sp}}\left(\cdot, p_{X}, \mathcal{W}\right)$ are reciprocal of each other

$$
E_{\mathrm{sp}}^{\prime}\left(R_{1}, p_{X}, \mathcal{W}\right)=\frac{1}{E_{\mathrm{sp}}^{\prime}\left(R_{2}, p_{X}, \mathcal{W}\right)} .
$$

The difference $d$ between two conjugate rates uniquely specifies them. We denote the smaller one by $R_{1}(d)$ and the larger one by $R_{2}(d)$, irrespective of the sign of $d$. Hence, $R_{1}(d) \leq R_{c r}\left(p_{X}, \mathcal{W}\right) \leq R_{2}(d)$, with equality when $d=0$. We also denote by $R^{\operatorname{conj}}\left(p_{X}, \mathcal{W}\right)$ the conjugate rate of $R$, as defined by (5.10). The conjugate rate always exists when $R$ is below the critical rate $R_{c r}\left(p_{X}, \mathcal{W}\right)$. If $R$ is above the critical rate and sufficiently large, $R^{\operatorname{conj}}\left(p_{X}, \mathcal{W}\right)$ may not exist. Instead of treating this case separately, we note that this case will be irrelevant because the conjugate rate always appears via the expression $\max \left\{R, R^{\operatorname{conj}}\left(p_{X}, \mathcal{W}\right)\right\}$ in our analysis. This maximum is equal to $R$ if $R>R_{c r}\left(p_{X}, \mathcal{W}\right)$ and is therefore always well defined.

The proofs of Propositions 5.1 and 5.3 and Lemma 5.4 below may be found in Appendices C-E; recall $F_{R, p_{X}, \mathcal{W}}(t)$ was defined in (4.5). The proof of Proposition 5.5 parallels that of Proposition 5.1(ii) and is therefore omitted.
Proposition 5.1: The suprema in (5.5) and (5.8) are respectively achieved by

$$
\begin{aligned}
F^{L *}(t) & =F_{R, p_{X}, \mathcal{W}}(t)+\Delta \\
& =\alpha-E_{\mathrm{sp}}\left(R+t, p_{X}, \mathcal{W}\right)
\end{aligned}
$$

and

$$
F^{*}(t)=\max \left(t, F^{L *}(t)\right)
$$

The resulting incorrect-message exponent is given by $E_{i}\left(R, p_{X}, \mathcal{W}\right)=\alpha$. The optimal solution is nonunique. In particular, for $t \leq 0$, one can replace $F^{L^{*}}(t)$ by the constant $F^{L *}(0)$ without effect on the error exponents.

The proof of Proposition 5.1 is quite simple and can be separated from the calculation of the error exponents. The main idea is this: If $G \preceq F$, we have

$$
E_{r, G}\left(R, p_{X}, \mathcal{W}\right) \leq E_{r, F}\left(R, p_{X}, \mathcal{W}\right) .
$$

Since $G^{-1} \succeq F^{-1}$, we also have

$$
E_{r, G^{-1}}\left(R, p_{X}, \mathcal{W}\right) \geq E_{r, F^{-1}}\left(R, p_{X}, \mathcal{W}\right) .
$$

Therefore, we seek $F^{*} \in \mathcal{F}^{L}\left(R, p_{X}, \mathcal{W}, \alpha\right)$ such that $F^{*} \preceq F$ for all $F \in \mathcal{F}^{L}\left(R, p_{X}, \mathcal{W}, \alpha\right)$. Such $F^{*}$, assuming it exists, necessarily achieves $E_{\emptyset}^{L}\left(R, p_{X}, \mathcal{W}, \alpha\right)$. The same procedure applies to $\mathcal{F}\left(R, p_{X}, \mathcal{W}, \alpha\right)$.

Corollary 5.2: If $R \geq I\left(p_{X}, \mathcal{W}\right)$, the thresholding rule $F^{L *}(t) \equiv \Delta$ of (5.1) is optimal, and the optimal error exponents are $E_{i}\left(R, p_{X}, \mathcal{W}, \alpha\right)=\Delta$ and $E_{\emptyset}^{L}\left(R, p_{X}, \mathcal{W}, \alpha\right)=0$.

Proof: Since $R \geq I\left(p_{X}, \mathcal{W}\right)$, we have $E_{\mathrm{sp}}\left(R, p_{X}, \mathcal{W}\right)=$ 0 . Hence, from (4.5), $F_{R, p_{X}, \mathcal{W}}(t) \equiv 0$ for all $t \geq 0$. Substituting into (5.11) establishes the optimality of the thresholding rule (5.1). The corresponding error exponents are obtained by minimizing (5.2) and (5.3) over $p_{Y \mid X} \in \mathcal{W}$.

The more elaborate case $R<I\left(p_{X}, \mathcal{W}\right)$ is addressed next.

Proposition 5.3: If $E_{\mathrm{sp}}\left(R, p_{X}, \mathcal{W}\right)$ is convex and differentiable in $R$, the optimal incorrect-message and erasure exponents are related as follows.

(i) For $\left|R^{\operatorname{conj}}\left(p_{X}, \mathcal{W}\right)-R\right|^{+} \leq \Delta \leq I\left(p_{X}, \mathcal{W}\right)-R$, we have

$$
\begin{aligned}
E_{i}\left(R, p_{X}, \mathcal{W}, \alpha\right) & =E_{\mathrm{sp}}\left(R, p_{X}, \mathcal{W}\right)+\Delta=\alpha \\
E_{\emptyset}^{L}\left(R, p_{X}, \mathcal{W}, \alpha\right) & =E_{\mathrm{sp}}\left(R+\Delta, p_{X}, \mathcal{W}\right) .
\end{aligned}
$$

(ii) The above exponents are also achieved using the penalty function $F(t)=\Delta+\lambda|t|^{+}$with

$$
-E_{\mathrm{sp}}^{\prime}\left(R, p_{X}, \mathcal{W}\right) \leq \lambda \leq \frac{1}{-E_{\mathrm{sp}}^{\prime}\left(R+\Delta, p_{X}, \mathcal{W}\right)} .
$$

(iii) If $R \leq R_{c r}\left(p_{X}, \mathcal{W}\right)$ and $0 \leq \Delta \leq R^{\operatorname{conj}}\left(p_{X}, \mathcal{W}\right)-R$, we have

$$
\begin{aligned}
E_{i}\left(R, p_{X}, \mathcal{W}, \alpha\right)= & E_{\mathrm{sp}}\left(R, p_{X}, \mathcal{W}\right)+\Delta=\alpha \\
E_{\emptyset}^{L}\left(R, p_{X}, \mathcal{W}, \alpha\right)= & E_{\mathrm{sp}}\left(R_{2}(\Delta), p_{X}, \mathcal{W}\right) \\
& +F_{R, p_{X}, \mathcal{W}}^{-1}\left(R_{1}(\Delta)-R\right) .
\end{aligned}
$$


(iv) If $R \leq R_{c r}\left(p_{X}, \mathcal{W}\right)$ and $R_{\infty}\left(p_{X}, \mathcal{W}\right)-R \leq \Delta \leq 0$, we have

$$
\begin{aligned}
E_{i}\left(R, p_{X}, \mathcal{W}, \alpha\right)= & E_{\mathrm{sp}}\left(R, p_{X}, \mathcal{W}\right)+\Delta=\alpha \\
E_{\emptyset}^{L}\left(R, p_{X}, \mathcal{W}, \alpha\right)= & E_{\mathrm{sp}}\left(R_{1}(\Delta), p_{X}, \mathcal{W}\right) \\
& +F_{R, p_{X}, \mathcal{W}}^{-1}\left(R_{2}(\Delta)-R\right) .
\end{aligned}
$$

Part (ii) of the proposition implies that not only is the optimal $F$ nonunique under the combinations of $\left(R, p_{X}, \mathcal{W}, \alpha\right)$ of Part (i), but also the Csiszár-Körner rule (2.10) is optimal for any $(\Delta, \lambda)$ in a certain range of values.

Also, while Proposition 5.3 provides simple expressions for the worst case error exponents over $\mathcal{W}$, the exponents for any specific channel $p_{Y \mid X} \in \mathcal{W}$ are obtained by substituting the function (5.12) and its inverse, respectively, into the minimization problem of (4.7). This problem does generally not admit a simple expression.

This leads us back to the question asked at the end of Section II, namely, when does the decoder pay no penalty for not knowing $p_{Y \mid X}$ ? Defining

$$
\underline{E}_{\mathrm{sp}}^{\prime}\left(R, p_{X}, \mathcal{W}\right) \triangleq \min _{p_{Y \mid X} \in \mathcal{W}} E_{\mathrm{sp}}^{\prime}\left(R, p_{X}, p_{Y \mid X}\right)
$$

and

$$
\bar{R}^{\mathrm{conj}}\left(p_{X}, \mathcal{W}\right) \triangleq \max _{p_{Y \mid X} \in \mathcal{W}} R^{\operatorname{conj}}\left(p_{X}, p_{Y \mid X}\right)
$$

we have the following lemma, whose proof appears in Appendix E.

Lemma 5.4:

$$
\begin{aligned}
E_{\mathrm{sp}}^{\prime}\left(R, p_{X}, \mathcal{W}\right) & \geq \underline{E}_{\mathrm{sp}}^{\prime}\left(R, p_{X}, \mathcal{W}\right) \\
\bar{R}^{\mathrm{conj}}\left(p_{X}, \mathcal{W}\right) & \geq R^{\mathrm{conj}}\left(p_{X}, \mathcal{W}\right)
\end{aligned}
$$

with equality if the same $p_{Y \mid X}$ minimizes $E_{\mathrm{sp}}\left(R, p_{X}, p_{Y \mid X}\right)$ at all rates.

Proposition 5.5: Assume that $R, p_{X}, \mathcal{W}, \Delta$, and $\lambda$ are such that

$$
\begin{aligned}
\left|\bar{R}^{\mathrm{conj}}\left(p_{X}, \mathcal{W}\right)-R\right|^{+} & \leq \Delta \leq I\left(p_{X}, \mathcal{W}\right)-R \\
-\underline{E}_{\mathrm{sp}}^{\prime}\left(R, p_{X}, \mathcal{W}\right) & \leq \lambda \leq \frac{1}{-\underline{E}_{\mathrm{sp}}^{\prime}\left(R+\Delta, p_{X}, \mathcal{W}\right)} .
\end{aligned}
$$

Then the pair of incorrect-message and erasure exponents

$$
\left\{E_{\mathrm{sp}}\left(R, p_{X}, p_{Y \mid X}\right)+\Delta, E_{\mathrm{sp}}\left(R+\Delta, p_{X}, p_{Y \mid X}\right)\right\}
$$

is universally attainable over $p_{Y \mid X} \in \mathcal{W}$ using the penalty function $F(t)=\Delta+\lambda|t|^{+}$, and equality holds in the erasure-exponent game of (3.11).

Proof: From (5.19) and (5.22), we have

$$
\begin{aligned}
\left|R^{\operatorname{conj}}\left(p_{X}, p_{Y \mid X}\right)-R\right|^{+} \leq \Delta \leq I\left(p_{X}, p_{Y \mid X}\right) & -R, \\
& \forall p_{Y \mid X} \in \mathcal{W} .
\end{aligned}
$$

Similarly, from (5.18) and (5.23), we have

$$
\begin{aligned}
&-E_{\mathrm{sp}}^{\prime}\left(R, p_{X}, p_{Y \mid X}\right) \leq \lambda \leq \frac{1}{-E_{\mathrm{sp}}^{\prime}\left(R+\Delta, p_{X}, p_{Y \mid X}\right)}, \\
& \forall p_{Y \mid X} \in \mathcal{W} .
\end{aligned}
$$

Then applying Proposition 5.3(ii) with the singleton $\left\{p_{Y \mid X}\right\}$ in place of $\mathcal{W}$ proves the claim.

The set of $(\Delta, \lambda)$ defined by (5.22), (5.23) is smaller than that of Proposition 5.3(i) but is not empty because $\underline{E}_{\mathrm{sp}}^{\prime}\left(R+\Delta, p_{X}, \mathcal{W}\right)$ tends to zero as $\Delta$ approaches the upper limit $I\left(p_{X}, \mathcal{W}\right)-R$. Thus, the universal exponents in (5.24) hold at least in the small erasure-exponent regime (where $\left.E_{\mathrm{sp}}\left(R+\Delta, p_{X}, p_{Y \mid X}\right) \rightarrow 0\right)$ and coincide with those derived by Forney [3, Theorem 3(a)] for symmetric channels, using maximum a posteriori probability (MAP) decoding. For symmetric channels, the same input distribution $p_{X}$ is optimal at all rates. ${ }^{3}$ Our rates are identical to his, i.e., the same optimal error exponents are achieved without knowledge of the channel.

\section{RELATIVE MinimaX}

When the compound class $\mathcal{W}$ is so large that $I\left(p_{X}, \mathcal{W}\right) \leq R$, we have seen from Corollary 5.2 that the simple thresholding rule $F(t) \equiv \Delta$ is optimal. Even if $I\left(p_{X}, \mathcal{W}\right)>R$, our minimax criterion (which seeks the worst case error exponents over the class $\mathcal{W}$ ) for designing $F$ might be a pessimistic one. This drawback can be alleviated to some extent using a relative minimax principle, see [12] and references therein. Our proposed approach is to define two functionals $\alpha\left(p_{Y \mid X}\right)$ and $\beta\left(p_{Y \mid X}\right)$ and the relative error exponents

$$
\begin{aligned}
& \Delta_{\alpha} E_{i}\left(R, p_{X}, p_{Y \mid X}, F\right) \triangleq E_{i}\left(R, p_{X}, p_{Y \mid X}, F\right)-\alpha\left(p_{Y \mid X}\right) \\
& \Delta_{\beta} E_{\emptyset}\left(R, p_{X}, p_{Y \mid X}, F\right) \triangleq E_{\emptyset}\left(R, p_{X}, p_{Y \mid X}, F\right)-\beta\left(p_{Y \mid X}\right) .
\end{aligned}
$$

Then solve the constrained optimization problem of (3.9) with the above functionals in place of $E_{i}\left(R, p_{X}, p_{Y \mid X}, F\right)-\alpha$ and $E_{\emptyset}\left(R, p_{X}, p_{Y \mid X}, F\right)$. It is reasonable to choose $\alpha\left(p_{Y \mid X}\right)$ and $\beta\left(p_{Y \mid X}\right)$ large for "good channels" and small for very noisy channels. Also, while $\alpha\left(p_{Y \mid X}\right)$ and $\beta\left(p_{Y \mid X}\right)$ could be the error exponents associated with some reference test, this is not a requirement. A possible choice is

$$
\begin{aligned}
& \alpha\left(p_{Y \mid X}\right)=\Delta \\
& \beta\left(p_{Y \mid X}\right)=E_{\mathrm{sp}}\left(R+\Delta, p_{X}, p_{Y \mid X}\right)
\end{aligned}
$$

which are the error exponents (5.2) and (5.3) corresponding to the thresholding rule $F(t) \equiv \Delta$. Another choice is

$$
\begin{aligned}
& \alpha\left(p_{Y \mid X}\right)=E_{\mathrm{sp}}\left(R, p_{X}, p_{Y \mid X}\right)+\Delta \\
& \beta\left(p_{Y \mid X}\right)=E_{\mathrm{sp}}\left(R+\Delta, p_{X}, p_{Y \mid X}\right)
\end{aligned}
$$

which are the "ideal" Forney exponents—achievable under the assumptions of Proposition 5.5(i).

The relative minimax problem is a simple extension of the minimax problem solved earlier. Define the following functions:

$$
\begin{aligned}
\Delta_{\alpha} E_{r, F}\left(R, p_{X}, \mathcal{W}\right)= & \min _{p_{Y \mid X} \in \mathcal{W}}\left[E_{r, F}\left(R, p_{X}, p_{Y \mid X}\right)\right. \\
& \left.-\alpha\left(p_{Y \mid X}\right)\right], \\
\Delta_{\alpha} E_{\mathrm{sp}}\left(R, p_{X}, \mathcal{W}\right)= & \min _{p_{Y \mid X} \in \mathcal{W}}\left[E_{\mathrm{sp}}\left(R, p_{X}, p_{Y \mid X}\right)\right. \\
& \left.-\alpha\left(p_{Y \mid X}\right)\right], \\
F_{R, p_{X}, \mathcal{W}, \alpha}(t)= & \Delta_{\alpha} E_{\mathrm{sp}}\left(R, p_{X}, \mathcal{W}\right) \\
& -\Delta_{\alpha} E_{\mathrm{sp}}\left(R+t, p_{X}, \mathcal{W}\right) .
\end{aligned}
$$

${ }^{3}$ Forney also studied the case $E_{\emptyset}(R)>E_{i}(R)$, which is not covered by our analysis. 
The function $F_{R, p_{X}, \mathcal{W}, \alpha}(t)$ of (6.5) is increasing and satisfies $F_{R, p_{X}, \mathcal{W}, \alpha}(0)=0$. The above functions $\Delta_{\alpha} E_{r, F}$ and $\Delta_{\alpha} E_{\mathrm{sp}}$ satisfy the following relationship:

$$
\begin{aligned}
& \Delta_{\alpha} E_{r, F}\left(R, p_{X}, \mathcal{W}\right) \\
& \stackrel{(\text { a) }}{=} \min _{p_{Y \mid X} \in \mathcal{W}}\left\{\operatorname { m i n } _ { R ^ { \prime } } \left[E_{\mathrm{sp}}\left(R^{\prime}, p_{X}, p_{Y \mid X}\right)\right.\right. \\
& \left.\left.+F\left(R^{\prime}-R\right)\right]-\alpha\left(p_{Y \mid X}\right)\right\} \\
& =\min _{R^{\prime}}\left\{\operatorname { m i n } _ { p _ { Y | X } \in \mathcal { W } } \left[E_{\mathrm{sp}}\left(R^{\prime}, p_{X}, p_{Y \mid X}\right)\right.\right. \\
& \left.\left.\left.-\alpha\left(p_{Y \mid X}\right)\right]+F\left(R^{\prime}-R\right)\right]\right\} \\
& \stackrel{(\mathrm{b})}{=} \min _{R^{\prime}}\left[\Delta_{\alpha} E_{\mathrm{sp}}\left(R^{\prime}, p_{X}, \mathcal{W}\right)+F\left(R^{\prime}-R\right)\right]
\end{aligned}
$$

where (a) is obtained from (4.7) and (6.3), and (b) from (6.4). Equation (6.6) is of the same form as (4.7), with $\Delta_{\alpha} E_{r, F}$ and $\Delta_{\alpha} E_{\mathrm{sp}}$ in place of $E_{r, F}-\alpha$ and $E_{\mathrm{sp}}-\alpha$, respectively.

Analogously to (5.4)-(5.6), the relative minimax for variablesize decoders is given by

$$
\begin{aligned}
& \Delta_{\beta} E_{\emptyset}^{L}(R, \mathcal{W}, \alpha) \\
& \quad=\max _{p_{X}} \sup _{F \in \mathcal{F}^{L}\left(R, p_{X}, \mathcal{W}, \alpha\right)} \Delta_{\beta} E_{r,\left|F^{-1}\right|^{+}}\left(R, p_{X}, \mathcal{W}\right)
\end{aligned}
$$

where the feasible set $\mathcal{F}^{L}\left(R, p_{X}, \mathcal{W}, \alpha\right)$ is the set of functions $F$ that satisfy

$$
\Delta_{\alpha} E_{r, F}\left(R, p_{X}, \mathcal{W}\right) \geq 0
$$

as well as the previous continuity and monotonicity conditions. The following proposition is analogous to Proposition 5.1.

Proposition 6.1: The supremum over $F$ in (6.7) is achieved by

$$
\begin{aligned}
F^{L *}(t) & =F_{R, p_{X}, \mathcal{W}, \alpha}(t)-\Delta_{\alpha} E_{\mathrm{sp}}\left(R, p_{X}, \mathcal{W}\right) \\
& =-\Delta_{\alpha} E_{\mathrm{sp}}\left(R+t, p_{X}, \mathcal{W}\right)
\end{aligned}
$$

independently of the choice of $\beta$. The relative minimax is given by

$$
\Delta_{\beta} E_{\emptyset}^{L}(R, \mathcal{W}, \alpha)=\max _{p_{X}} \Delta_{\beta} E_{r,\left|\left(F^{L *}\right)^{-1}\right|}\left(R, p_{X}, \mathcal{W}\right)
$$

Proof: The proof exploits the same monotonicity property $\left(E_{r, F} \leq E_{r, G}\right.$ for $F \preceq G$ ) that was used to derive the optimal $F$ in (5.12). The supremum over $F$ is obtained by following the steps of the proof of Proposition 5.1, substituting $\Delta_{\alpha} E_{r, F}$, $\Delta_{\alpha} E_{\mathrm{sp}}$, and $F_{R, p_{X}, \mathcal{W}, \alpha}$ for $E_{r, F}-\alpha, E_{\mathrm{sp}}-\alpha$, and $F_{R, p_{X}, \mathcal{W}}$, respectively. The relative minimax is obtained by substituting the optimal $F$ into (6.7).

We would like to know how much influence the reference function $\alpha$ has on the optimal $F$. For the "Forney reference exponent function" $\alpha$ of (6.1), we obtain the optimal $F$ from (6.8) and (6.4):

$$
\begin{gathered}
F^{L *}(t)=-\min _{p_{Y \mid X} \in \mathcal{W}}\left[E_{\mathrm{sp}}\left(R+t, p_{X}, p_{Y \mid X}\right)-\alpha\left(p_{Y \mid X}\right)\right] \\
=-\min _{p_{Y \mid X} \in \mathcal{W}}\left[E_{\mathrm{sp}}\left(R+t, p_{X}, p_{Y \mid X}\right)\right. \\
\left.-E_{\mathrm{sp}}\left(R, p_{X}, p_{Y \mid X}\right)-\Delta\right]
\end{gathered}
$$

$$
\begin{aligned}
& =\Delta+\max _{p_{Y \mid X} \in \mathcal{W}}\left[E_{\mathrm{sp}}\left(R, p_{X}, p_{Y \mid X}\right)\right. \\
& \left.\quad-E_{\mathrm{sp}}\left(R+t, p_{X}, p_{Y \mid X}\right)\right] \\
& =\Delta+\max _{p_{Y \mid X} \in \mathcal{W}} F_{R, p_{X}, p_{Y \mid X}}(t) .
\end{aligned}
$$

Interestingly, the maximum above is often achieved by the cleanest channel in $\mathcal{W}$-for which $E_{\mathrm{sp}}\left(R, p_{X}, p_{Y \mid X}\right)$ is large and $E_{\mathrm{sp}}\left(R+t, p_{X}, p_{Y \mid X}\right)$ falls off rapidly as $t$ increases. This stands in contrast to (5.12) which may be written as

$$
\begin{aligned}
F^{L *}(t)= & \alpha-\min _{p_{Y \mid X} \in \mathcal{W}} E_{\mathrm{sp}}\left(R+t, p_{X}, p_{Y \mid X}\right) \\
= & \Delta+\min _{p_{Y \mid X} \in \mathcal{W}} E_{\mathrm{sp}}\left(R, p_{X}, p_{Y \mid X}\right) \\
& -\min _{p_{Y \mid X} \in \mathcal{W}} E_{\mathrm{sp}}\left(R+t, p_{X}, p_{Y \mid X}\right) .
\end{aligned}
$$

In (6.10), the minima are achieved by the noisiest channel at rates $R$ and $R+t$, respectively. Also note that $F^{L *}(t)$ from (6.9) is uniformly larger than $F^{L *}(t)$ from (6.10) and thus results in larger incorrect-message exponents.

For $R>I\left(p_{X}, \mathcal{W}\right)$, Corollary 5.2 has shown that the minimax criterion is maximized by the thresholding rule $F(t)=\Delta$ which yields

$$
\begin{aligned}
& E_{i}\left(R, p_{X}, p_{Y \mid X}\right)=\Delta \\
& E_{\emptyset}\left(R, p_{X}, p_{Y \mid X}\right)=E_{\mathrm{sp}}\left(R+\Delta, p_{X}, p_{Y \mid X}\right), \quad \forall p_{Y \mid X} .
\end{aligned}
$$

The relative minimax criterion based on $\alpha\left(p_{Y \mid X}\right)$ of (6.1) yields a higher $E_{i}\left(R, p_{X}, p_{Y \mid X}\right)$ for good channels and this is counterbalanced by a lower $E_{\emptyset}\left(R, p_{X}, p_{Y \mid X}\right)$. Thus, the primary advantage of the relative minimax approach is that $\alpha\left(p_{Y \mid X}\right)$ can be chosen to more finely balance the error exponents across the range of channels of interest.

\section{COMPOUND BINARY-SYMMETRIC CHANNEL}

We have evaluated the incorrect-message and erasure exponents of (5.24) for the compound BSC with crossover probability $\rho \in\left[\rho_{\min }, \rho_{\max }\right]$, where $0<\rho_{\min }<\rho_{\max } \leq \frac{1}{2}$. The class $\mathcal{W}$ may be identified with the interval $\left[\rho_{\min }, \rho_{\max }\right]$, where $\rho_{\min }$ and $\rho_{\max }$ correspond to the cleanest and noisiest channels in $\mathcal{W}$, respectively. Denote by $h_{2}(\rho) \triangleq-\rho \log \rho-(1-\rho) \log (1-\rho)$ the binary entropy function, by $h_{2}^{-1}(\cdot)$ the inverse of that function over the range $\left[0, \frac{1}{2}\right]$, and by $p_{\rho}$ the Bernoulli pmf with parameter $\rho$.

Capacity of the BSC is given by $C(\rho)=1-h_{2}(\rho)$, and the sphere packing exponent by [1, p. 195]

$$
\begin{aligned}
E_{\mathrm{sp}}(R, \rho) & =D\left(p_{\rho_{R}} \| p_{\rho}\right) \\
& =\rho_{R} \log \frac{\rho_{R}}{\rho}+\left(1-\rho_{R}\right) \log \frac{1-\rho_{R}}{1-\rho}, \\
& 0 \leq R \leq C(\rho)
\end{aligned}
$$

where $\rho_{R}=h_{2}^{-1}(1-R) \geq \rho$. The optimal input distribution $p_{X}$ is uniform at all rates and will be omitted from the list of arguments of the functions $E_{r, F}, E_{\mathrm{sp}}$, and $F_{R}$ below. The critical rate is

$$
R_{c r}(\rho)=1-h_{2}\left(\frac{1}{1+\sqrt{1 / \rho^{2}-1}}\right)
$$

and $E_{\mathrm{sp}}(0, \rho)=-\log \sqrt{4 \rho(1-\rho)}$. 
The capacity and sphere-packing exponent for the compound BSC are respectively given by $C(\mathcal{W})=C\left(\rho_{\max }\right)$ and

$$
\begin{aligned}
E_{\mathrm{sp}}(R, \mathcal{W}) & =\min _{\rho_{\min } \leq \rho \leq \rho_{\max }} E_{\mathrm{sp}}(R, \rho) \\
& =E_{\mathrm{sp}}\left(R, \rho_{\max }\right) .
\end{aligned}
$$

For $R \geq C(\mathcal{W})$, the optimal $F$ is the thresholding rule of (5.1), and (5.2), (5.3) yield

$$
E_{i}(R, \rho)=\Delta \quad \text { and } \quad E_{\emptyset}(R, \rho)=E_{\mathrm{sp}}(R+\Delta, \rho) .
$$

In the remainder of this section, we assume $R<C(\mathcal{W})$, in which case $E_{\mathrm{sp}}(R, \mathcal{W})>0$.

Optimal F: For any $0 \leq \Delta \leq C(\rho)-R$, we have $\rho \leq$ $\rho_{R+\Delta} \leq \rho_{R} \leq \frac{1}{2}$. From (7.1) we have

$$
\begin{aligned}
F_{R, \rho}(t)= & E_{\mathrm{sp}}(R, \rho)-E_{\mathrm{sp}}(R+t, \rho) \\
= & D\left(p_{\rho_{R}} \| p_{\rho}\right)-D\left(p_{\rho_{R+t}} \| p_{\rho}\right) \\
= & h_{2}\left(\rho_{R}\right)-h_{2}\left(\rho_{R+t}\right)+\left(\rho_{R}-\rho_{R+t}\right) \\
& \times\left(\log \frac{1}{\rho}-\log \frac{1}{1-\rho}\right) \\
= & h_{2}\left(\rho_{R}\right)-h_{2}\left(\rho_{R+t}\right)+\left(\rho_{R}-\rho_{R+t}\right) \\
& \times \log \left(\frac{1}{\rho}-1\right), \quad t \geq 0
\end{aligned}
$$

which is a decreasing function of $\rho$.

Evaluating the optimal $F$ from (5.12), we have

$$
\begin{aligned}
F^{L *}(t) & =\Delta+F_{R, \mathcal{W}}(t) \\
& =\Delta+E_{\mathrm{sp}}(R, \mathcal{W})-E_{\mathrm{sp}}(R+t, \mathcal{W}) \\
& =\Delta+E_{\mathrm{sp}}\left(R, \rho_{\max }\right)-E_{\mathrm{sp}}\left(R+t, \rho_{\max }\right) \\
& =\Delta+F_{R, \rho_{\max }}(t), \quad t \geq 0 .
\end{aligned}
$$

Observe that the optimal $F$ is determined by the noisiest channel $\left(\rho_{\max }\right)$ and does not depend at all on $\rho_{\min }$.

This contrasts with the relative minimax criterion with $\alpha\left(p_{Y \mid X}\right)$ of (6.1), where evaluation of the optimal $F$ from (6.9) yields

$$
\begin{aligned}
F^{L *}(t) & =\Delta+\max _{\rho_{\min } \leq \rho \leq \rho_{\max }} F_{R, \rho}(t) \\
& =\Delta+F_{R, \rho_{\min }}(t), \quad t \geq 0
\end{aligned}
$$

which is determined by the cleanest channel $\left(\rho_{\min }\right)$ and does not depend on $\rho_{\max }$.

Optimal Error Exponents: Fig. 2 displays $E_{\emptyset}(R, \mathcal{W})$ and $E_{i}(R, \mathcal{W})$ when $\rho_{\max }=0.1$ (the value of $\rho_{\min }$ is immaterial for the minimax criterion here), $R=0.1<C\left(\rho_{\max }\right) \approx$ 0.53 , and the exponent tradeoff parameter $\Delta$ ranges from $-R$ to $C\left(\rho_{\max }\right)-R$. The resulting exponents are obtained from Proposition 5.3, and the existence of the three regimes, respectively described by (5.14), (5.16), and (5.17), may be seen in the figure.

Next we derive expressions for $E_{\mathrm{sp}}^{\prime}(R, \mathcal{W})$ and $\underline{E}_{\mathrm{sp}}^{\prime}(R, \mathcal{W})$. The derivations are simplified if instead of working with the crossover probability $\rho$, we use the following reparameterization:

$$
\mu \triangleq \rho^{-1}-1, \quad \mu_{\max } \triangleq \rho_{\min }^{-1}-1, \quad \mu_{\min } \triangleq \rho_{\max }^{-1}-1
$$

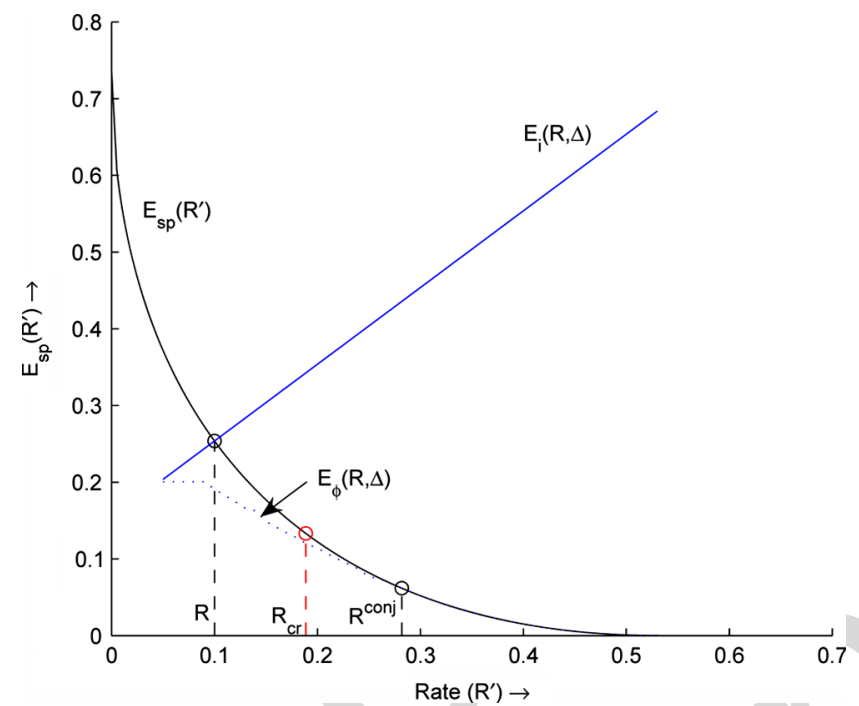

Fig. 2. Erasure and incorrect-message exponents $E_{\emptyset}(R, \mathcal{W})$ and $E_{i}(R, \mathcal{W})$ when $R=0.1$ and $\mathcal{W}$ is the family of BSCs with crossover probability $\rho \leq$ $\rho_{\max }=0.1$. The compound channel capacity is $C\left(\rho_{\max }\right) \approx 0.53$.

and

$\mu_{R} \triangleq \rho_{R}^{-1}-1=\frac{1}{h_{2}^{-1}(1-R)}-1 \Leftrightarrow R=1-h_{2}\left(\frac{1}{1+\mu}\right)$

where $\mu_{R}$ increases monotonically from 1 to $\infty$ as $R$ increases from 0 to 1 . With this notation, we have $\rho=\frac{1}{1+\mu}$ and $\mu_{\max } \geq$ $\mu \geq \mu_{R+\Delta} \geq \mu_{R} \geq 1$. Also

$$
\begin{aligned}
\frac{d R}{d \rho_{R}} & =-\frac{d h_{2}\left(\rho_{R}\right)}{d \rho_{R}}=-\frac{\log \mu_{R}}{\ln 2} \\
\frac{d E_{\mathrm{sp}}(R, \rho)}{d \rho_{R}} & =\frac{\log \mu-\log \mu_{R}}{\ln 2}
\end{aligned}
$$

$-E_{\mathrm{sp}}^{\prime}(R, \rho)=\frac{d E_{\mathrm{sp}}(R, \rho) / d \rho_{R}}{-d R / d \rho_{R}}=\frac{\log \mu}{\log \mu_{R}}-1=\frac{\log \mu / \mu_{R}}{\log \mu_{R}}$.

From (5.18) and (7.3), we obtain

$$
-\underline{E}_{\mathrm{sp}}^{\prime}(R, \mathcal{W})=-\min _{\rho_{\min } \leq \rho \leq \rho_{\max }} E_{\mathrm{sp}}^{\prime}(R, \rho)=\frac{\log \mu_{\max } / \mu_{R}}{\log \mu_{R}} .
$$

Observe that the minimizing $\rho$ is $\rho_{\min }$, i.e., the cleanest channel in $\mathcal{W}$. In contrast, from (7.2), we have

$$
\begin{aligned}
-E_{\mathrm{sp}}^{\prime}(R, \mathcal{W}) & =-E_{\mathrm{sp}}^{\prime}\left(R, \rho_{\max }\right) \\
& =\frac{\log \mu_{\min } / \mu_{R}}{\log \mu_{R}}<-\underline{E}_{\mathrm{sp}}^{\prime}(R, \mathcal{W})
\end{aligned}
$$

which is determined by the noisiest channel $\left(\rho_{\max }\right)$.

Conditions for Universality: Next we evaluate $\bar{R}^{\text {conj }}(\mathcal{W})$ from (5.19). For a given $\rho$, the conjugate rate of $R$ is obtained from (7.3)

$$
\begin{aligned}
-E_{\mathrm{sp}}^{\prime}(R, \rho) & =\frac{1}{-E_{\mathrm{sp}}^{\prime}\left(R^{\mathrm{conj}}, \rho\right)} \\
\frac{\log \left(\mu / \mu_{R}\right)}{\log \mu_{R}} & =\frac{\log \mu_{R^{\mathrm{conj}}}}{\log \left(\mu / \mu_{R^{\mathrm{conj}}}\right)}
\end{aligned}
$$


hence

$$
\begin{aligned}
& \mu_{R^{\mathrm{conj}}}=\frac{\mu}{\mu_{R}} \\
& R^{\mathrm{conj}}(\mu)=1-h_{2}\left(\frac{1}{1+\mu / \mu_{R}}\right) \\
& \bar{R}^{\text {conj }}(\mathcal{W})=\max _{\mu_{\min } \leq \mu \leq \mu_{\max }} R^{\text {conj }}(\mu) \\
& =R^{\mathrm{conj}}\left(\mu_{\max }\right) \text {. }
\end{aligned}
$$

From (5.10) and (7.2), we have

$$
R^{\mathrm{conj}}(\mathcal{W})=R^{\mathrm{conj}}\left(\mu_{\min }\right) .
$$

Analogously to (7.5), observe that both $\bar{R}^{\text {conj }}(\mathcal{W})$ and $R^{\operatorname{conj}}(\mathcal{W})$ are determined by the cleanest and noisiest channels in $\mathcal{W}$, respectively.

We can now evaluate the conditions of Proposition 5.5, under which $F(t)=\Delta+\lambda|t|^{+}$is universal (subject to conditions on $\Delta$ and $\lambda$ ). In (5.22), $\Delta$ must satisfy (7.6) shown at the bottom of the page. The left side is zero if $\mu_{\max } \leq \mu_{R}^{2}$. If $\mu_{\max }>\mu_{R}^{2}$, the argument of $|\cdot|^{+}$is positive, and we need $\mu_{\max } / \mu_{R}<\mu_{\min }$ to ensure that the left side is lower than the right side. Hence, there exists a nonempty range of values of $\Delta$ satisfying (7.6) if and only if

$$
\mu_{R} \geq \min \left\{\sqrt{\mu_{\max }}, \frac{\mu_{\max }}{\mu_{\min }}\right\}
$$

which may also be written as

$$
\mu_{\max } \leq \max \left\{\mu_{R}^{2}, \mu_{R} \mu_{\min }\right\} .
$$

Next, substituting (7.4) into (5.23), we obtain the following condition for $\lambda$ :

$$
\frac{\log \mu_{\max } / \mu_{R}}{\log \mu_{R}} \leq \lambda \leq \frac{\log \mu_{R+\Delta}}{\log \mu_{\max } / \mu_{R+\Delta}} .
$$

This equation has a solution if and only if the left side does not exceed the right side, i.e.,

$$
\mu_{\max } \leq \mu_{R} \mu_{R+\Delta}
$$

or, equivalently, $\rho_{\min } \geq\left(1+\mu_{R} \mu_{R+\Delta}\right)^{-1}$. Since $\mu_{R}$ is an increasing function of $R$, the larger the values of $R$ and $\Delta$, the lower the value of $\rho_{\min }$ for which the universality property still holds.

If equality holds in (7.8), the only feasible value of $\lambda$ is

$$
\lambda=\frac{\log \mu_{R+\Delta}}{\log \mu_{R}} \geq 1
$$

This value of $\lambda$ remains feasible if (7.8) holds with strict inequality.

\section{DISCUSSION}

The $\mathcal{F}$-MMI decision rule of (3.1) is a generalization of Csiszár and Körner's MMI decoder with erasure option. The weighting function $F$ in (3.1) can be optimized in an asymptotic Neyman-Pearson sense given a compound class of channels $\mathcal{W}$. An explicit formula has been derived in terms of the sphere-packing exponent function for $F$ that maximizes the erasure exponent subject to a constraint on the incorrect-message exponent. The optimal $F$ is generally nonunique but agrees with existing designs in special cases of interest.

In particular, Corollary 5.2 shows that the simple thresholding rule $F(t) \equiv \Delta$ is optimal if $R \geq I\left(p_{X}, \mathcal{W}\right)$, i.e., when the transmission rate cannot be reliably supported by the worst channel in $\mathcal{W}$. When $R<I\left(p_{X}, \mathcal{W}\right)$, Proposition 5.5 shows that for small erasure exponents, our expressions for the optimal exponents coincide with those derived by Forney [3] for symmetric channels, where the same input distribution $p_{X}$ is optimal at all rates. In this regime, Csiszár and Körner's rule $F(t)=$ $\Delta+\lambda|t|^{+}$is also universal under some conditions on the parameter pair $(\Delta, \lambda)$. It is also worth noting that while suboptimal, the design $F(t)=\Delta+t$ yields an empirical version of Forney's simple decision rule (2.5).

Previous work [6] using a different universal decoder had shown that Forney's exponents can be matched in the special case where undetected-error and erasure exponents are equal (corresponding to $T=0$ in Forney's rule (2.4)). Our results show that this property extends beyond this special case, albeit not everywhere.

Another analogy between Forney's suboptimal decision rule (2.5) and ours (3.1) is that the former is based on the likelihood score of each candidate message and its highest scoring competitor, and ours uses empirical mutual information instead of likelihood. Our results imply that (2.5) is optimal (in terms of error exponents) in the special regime identified above.

The relative minimax criterion of Section VI is attractive when the compound class $\mathcal{W}$ is broad (or difficult to pick) as it allows finer tuning of the error exponents for different channels in $\mathcal{W}$. The class $\mathcal{W}$ could conceivably be as large as $\mathcal{P}_{Y \mid X}$, the set of all DMCs. Depending on the choice of the reference exponents and the class $\mathcal{W}$, the design of $F$ may be determined by the least noisy channel in $\mathcal{W}$ instead of the noisiest one.

Finally, we have extended our framework to decoding for compound MACs. Those results will be presented elsewhere.

$$
\begin{gathered}
\left|\bar{R}^{\operatorname{conj}}(\mathcal{W})-R\right|^{+} \leq \Delta \leq C(\mathcal{W})-R \\
\mid R^{\operatorname{conj}\left(\mu_{\max }\right)-\left.R\right|^{+}} \leq \Delta \leq C\left(\mu_{\min }\right)-R \\
\left|h_{2}\left(\frac{1}{1+\mu_{R}}\right)-h_{2}\left(\frac{1}{1+\mu_{\max } / \mu_{R}}\right)\right|^{+} \leq \Delta \leq h_{2}\left(\frac{1}{1+\mu_{R}}\right)-h_{2}\left(\frac{1}{1+\mu_{\min }}\right) .
\end{gathered}
$$




\section{APPENDIX A \\ PROOF OF PROPOSITION 4.1}

(i) follows from (4.3), restated below

$$
\begin{aligned}
E_{r, F}\left(R, p_{X}, p_{Y \mid X}\right)=\min _{\tilde{p}_{Y \mid X}}[ & D\left(\tilde{p}_{Y \mid X} \| p_{Y \mid X} \mid p_{X}\right) \\
& \left.+F\left(I\left(p_{X}, \tilde{p}_{Y \mid X}\right)-R\right)\right]
\end{aligned}
$$

and the fact that $F$ is increasing. Indeed, let $\tilde{p}_{Y \mid X}^{[R]}$ achieve the minimum of (A.1), at rate $R$, and choose $R<R^{\prime} \leq$ $I\left(p_{X}, p_{Y \mid X}\right)$. Then evaluating (A.1) at $R^{\prime}$ we have

$$
\begin{aligned}
E_{r, F} & \left(R^{\prime}, p_{X}, p_{Y \mid X}\right) \\
& \leq D\left(\tilde{p}_{Y \mid X}^{[R]} \| p_{Y \mid X} \mid p_{X}\right)+F\left(I\left(p_{X}, \tilde{p}_{Y \mid X}^{[R]}\right)-R^{\prime}\right) \\
& <D\left(\tilde{p}_{Y \mid X}^{[R]} \| p_{Y \mid X} \mid p_{X}\right)+F\left(I\left(p_{X}, \tilde{p}_{Y \mid X}^{[R]}\right)-R\right) \\
& =\min _{\tilde{p}_{Y \mid X}}\left[D\left(\tilde{p}_{Y \mid X} \| p_{Y \mid X} \mid p_{X}\right)+F\left(I\left(p_{X}, \tilde{p}_{Y \mid X}\right)-R\right)\right] \\
& =E_{r, F}\left(R, p_{X}, p_{Y \mid X}\right) .
\end{aligned}
$$

(ii) holds for the same reason as above.

(iii) Since the function $E_{\mathrm{sp}}\left(R, p_{X}, p_{Y \mid X}\right)$ is decreasing in $R$ for $R \leq I\left(p_{X}, p_{Y \mid X}\right)$, it follows from (4.1) that

$$
\begin{array}{r}
E_{\mathrm{Sp}}\left(R, p_{X}, p_{Y \mid X}\right)=\min _{\tilde{p}_{Y \mid X}: I\left(p_{X}, \tilde{p}_{Y \mid X}\right)=R} D\left(\tilde{p}_{Y \mid X} \| p_{Y \mid X} \mid p_{X}\right), \\
\forall R \leq I\left(p_{X}, p_{Y \mid X}\right) . \quad \text { (A.2) }
\end{array}
$$

For any $\tilde{p}_{Y \mid X}$ such that $I\left(p_{X}, \tilde{p}_{Y \mid X}\right) \geq I\left(p_{X}, p_{Y \mid X}\right)$, we have $F\left(I\left(p_{X}, \tilde{p}_{Y \mid X}\right)-R\right) \geq F\left(I\left(p_{X}, p_{Y \mid X}\right)-R\right)$ because $F$ is nondecreasing. The minimum of $D+F$ in (A.1) over all such $\tilde{p}_{Y \mid X}$ is therefore achieved by $\tilde{p}_{Y \mid X}=p_{Y \mid X}$. Thus, $\tilde{p}_{Y \mid X}$ that achieves the unconstrained minimum in (A.1) must satisfy $I\left(p_{X}, \tilde{p}_{Y \mid X}\right) \leq I\left(p_{X}, p_{Y \mid X}\right)$. Hence, we have the equation at the bottom of the page, where (a) is due to (A.2), and (b) holds because $E_{\mathrm{sp}}\left(R^{\prime}, p_{X}, p_{Y \mid X}\right)=0$ for $R^{\prime} \geq I\left(p_{X}, p_{Y \mid X}\right)$ and $F$ is nondecreasing.

(iv) The claim follows directly from the definitions (4.2) and (4.4), e.g., (i) is proved by observing that $E_{r, F}\left(R, p_{X}, p_{Y \mid X}\right)<$ $E_{r, F}\left(R^{\prime}, p_{X}, p_{Y \mid X}\right)$ implies

$$
\begin{aligned}
E_{r, F}\left(R, p_{X}, \mathcal{W}\right) & =\min _{p_{Y \mid X} \in \mathcal{W}} E_{r, F}\left(R, p_{X}, p_{Y \mid X}\right) \\
& <\min _{p_{Y \mid X} \in \mathcal{W}} E_{r, F}\left(R^{\prime}, p_{X}, p_{Y \mid X}\right) \\
& =E_{r, F}\left(R^{\prime}, p_{X}, \mathcal{W}\right)
\end{aligned}
$$

for all $R \leq I\left(p_{X}, \mathcal{W}\right)$. Property (ii) is immediate as well. Property (iii) holds because

$$
\begin{aligned}
E_{r, F} & \left(R, p_{X}, \mathcal{W}\right) \\
& =\min _{p_{Y \mid X} \in \mathcal{W}} \min _{R^{\prime}}\left[E_{\mathrm{sp}}\left(R^{\prime}, p_{X}, p_{Y \mid X}\right)+F\left(R^{\prime}-R\right)\right] \\
& =\min _{R^{\prime}}\left[\min _{p_{Y \mid X} \in \mathcal{W}} E_{\mathrm{sp}}\left(R^{\prime}, p_{X}, p_{Y \mid X}\right)+F\left(R^{\prime}-R\right)\right] \\
& =\min _{R^{\prime}}\left[E_{\mathrm{sp}}\left(R^{\prime}, p_{X}, \mathcal{W}\right)+F\left(R^{\prime}-R\right)\right] .
\end{aligned}
$$

APPENDIX B

PROOF OF PROPOSITION 4.2

Given the pmf $p_{X}$, choose any type $p_{\boldsymbol{x}}$ such that $\max _{x \in \mathcal{X}}\left|p_{\boldsymbol{x}}(x)-p_{X}(x)\right| \leq \frac{|\mathcal{X}|}{N} \cdot{ }^{4}$ Define

$$
\begin{aligned}
& E_{r, F, N}\left(R, p_{\boldsymbol{x}}, p_{Y \mid X}\right) \\
& \quad=\min _{p_{\boldsymbol{y} \mid \boldsymbol{x}}}\left[D\left(p_{\boldsymbol{y} \mid \boldsymbol{x}} \| p_{Y|X|} \mid p_{\boldsymbol{x}}\right)+F(I(\boldsymbol{x} ; \boldsymbol{y})-R)\right]
\end{aligned}
$$

and

$$
E_{s p, N}\left(R, p_{\boldsymbol{x}}, p_{Y \mid X}\right)=\min _{p_{\boldsymbol{y} \mid x}: I(\boldsymbol{x} ; \boldsymbol{y}) \leq R} D\left(p_{\boldsymbol{y} \mid \boldsymbol{x}}|| p_{Y \mid X} \mid p_{\boldsymbol{x}}\right)
$$

which differ from (4.3) and (4.1) in that the minimization is performed over conditional types instead of general conditional pmfs. We have

$$
\begin{aligned}
\lim _{N \rightarrow \infty} E_{r, F, N}\left(R, p_{\boldsymbol{x}}, p_{Y \mid X}\right) & =E_{r, F}\left(R, p_{X}, p_{Y \mid X}\right) \\
\lim _{N \rightarrow \infty} E_{s p, N}\left(R, p_{\boldsymbol{x}}, p_{Y \mid X}\right) & =E_{\mathrm{sp}}\left(R, p_{X}, p_{Y \mid X}\right)
\end{aligned}
$$

by continuity of the divergence functional and of $F$ in the region where the minimand $D+F$ is finite.

We will use the following two standard inequalities.

1) Given an arbitrary sequence $\boldsymbol{y}$, draw $\boldsymbol{x}^{\prime}$ independently of $\boldsymbol{y}$ and uniformly over a fixed type class $T_{\boldsymbol{x}}$. Then [1]

$$
\operatorname{Pr}\left[T_{\boldsymbol{x}^{\prime} \mid \boldsymbol{y}}\right]=\frac{\left|T_{\boldsymbol{x}^{\prime} \mid \boldsymbol{y}}\right|}{\left|T_{\boldsymbol{x}}\right|}=\frac{\left|T_{\boldsymbol{x}^{\prime} \mid \boldsymbol{y}}\right|}{\left|T_{\boldsymbol{x}^{\prime}}\right|} \doteq 2^{-N I\left(\boldsymbol{x}^{\prime} ; \boldsymbol{y}\right)}
$$

Hence, for any $0 \leq \nu \leq H\left(p_{\boldsymbol{x}}\right)$

$$
\operatorname{Pr}\left[I\left(\boldsymbol{x}^{\prime} ; \boldsymbol{y}\right) \geq \nu\right]=\sum_{T_{\boldsymbol{x}^{\prime} \mid \boldsymbol{y}}} \operatorname{Pr}\left[T_{\boldsymbol{x}^{\prime} \mid \boldsymbol{y}}\right] \mathbb{1}_{\left\{I\left(\boldsymbol{x}^{\prime} ; \boldsymbol{y}\right) \geq \nu\right\}}
$$

${ }^{4}$ For instance, truncate each $p_{X}(x)$ down to the nearest integer multiple of $1 / N$ and add $a / N$ to the smallest resulting value to obtain $p_{\boldsymbol{x}}(x), x \in \mathcal{X}$, summing to one. $a$ is an integer in the range $\{0,1, \ldots,|\mathcal{X}|-1\}$.

$$
\begin{aligned}
E_{r, F}\left(R, p_{X}, p_{Y \mid X}\right) & =\min _{\tilde{p}_{Y \mid X}: I\left(p_{X}, \tilde{p}_{Y \mid X}\right) \leq I\left(p_{X}, p_{Y \mid X}\right)}\left[D\left(\tilde{p}_{Y \mid X} \| p_{Y \mid X} \mid p_{X}\right)+F\left(I\left(p_{X}, \tilde{p}_{Y \mid X}\right)-R\right)\right] \\
& =\min _{R^{\prime} \leq I\left(p_{X}, p_{Y \mid X}\right)} \tilde{p}_{Y \mid X}: I\left(p_{X}, \tilde{p}_{Y \mid X}\right)=R^{\prime} \\
& \stackrel{(\text { a) }}{=} \min _{R^{\prime} \leq I\left(p_{X}, p_{Y \mid X}\right)}\left[E_{\mathrm{sp}}\left(R^{\prime}, p_{X}, p_{Y \mid X}\right)+F\left(R^{\prime}-R\right)\right] \\
& \stackrel{\text { b) }}{=} \min _{R^{\prime}}\left[E_{\mathrm{sp}}\left(R^{\prime}, p_{X}, p_{Y \mid X}\right)+F\left(p_{X}\right)+F\left(R^{\prime}-R\right)\right]
\end{aligned}
$$




$$
\begin{aligned}
& \doteq \sum_{T_{\boldsymbol{x}^{\prime} \mid \boldsymbol{y}}} 2^{-N I\left(\boldsymbol{x}^{\prime} ; \boldsymbol{y}\right)} \mathbb{1}_{\left\{I\left(\boldsymbol{x}^{\prime} ; \boldsymbol{y}\right) \geq \nu\right\}} \\
& \stackrel{(\mathrm{a})}{\doteq} \max _{T_{\boldsymbol{x}^{\prime} \mid \boldsymbol{y}}} 2^{-N I\left(\boldsymbol{x}^{\prime} ; \boldsymbol{y}\right)} \mathbb{1}_{\left\{I\left(\boldsymbol{x}^{\prime} ; \boldsymbol{y}\right) \geq \nu\right\}} \\
& \doteq 2^{-N \nu}
\end{aligned}
$$

where (a) holds because the number of types is polynomial in $N$. For $\nu>H\left(p_{\boldsymbol{x}}\right)$ we have $\operatorname{Pr}\left[I\left(\boldsymbol{x}^{\prime} ; \boldsymbol{y}\right) \geq \nu\right]=0$.

2) Given an arbitrary sequence $\boldsymbol{x}$, draw $\boldsymbol{y}$ from the conditional $\operatorname{pmf} p_{Y \mid X}^{N}(\cdot \mid \boldsymbol{x})$. We have [1]

$$
\operatorname{Pr}\left[T_{\boldsymbol{y} \mid \boldsymbol{x}}\right] \doteq 2^{-N D\left(p_{\boldsymbol{y} \mid \boldsymbol{x}} \| p_{Y \mid X} \mid p_{\boldsymbol{x}}\right)} .
$$

Then, for any $\nu>0$

$$
\begin{aligned}
\operatorname{Pr}[I(\boldsymbol{x} ; \boldsymbol{y}) \leq \nu]= & \sum_{T_{\boldsymbol{y} \mid \boldsymbol{x}}} \operatorname{Pr}\left[T_{\boldsymbol{y} \mid \boldsymbol{x}}\right] \\
& \times \mathbb{1}_{\{I(\boldsymbol{x} ; \boldsymbol{y}) \leq \nu\}} \\
\doteq & \sum_{p_{\boldsymbol{y} \mid \boldsymbol{x}}} 2^{-N D\left(p_{\boldsymbol{y} \mid \boldsymbol{x}} \| p_{Y \mid X} \mid p_{\boldsymbol{x}}\right)} \\
& \times \mathbb{1}_{\{I(\boldsymbol{x} ; \boldsymbol{y}) \leq \nu\}} \\
\doteq & \max _{p_{\boldsymbol{y} \mid \boldsymbol{x}}} 2^{-N D\left(p_{\boldsymbol{y} \mid \boldsymbol{x}} \| p_{Y \mid X} \mid p_{\boldsymbol{x}}\right)} \\
& \times \mathbb{1}_{\{I(\boldsymbol{x} ; \boldsymbol{y}) \leq \nu\}} \\
= & \max _{p_{\boldsymbol{y} \mid \boldsymbol{x}}: I(\boldsymbol{x} ; \boldsymbol{y}) \leq \nu} 2^{-N D\left(p_{\boldsymbol{y} \mid \boldsymbol{x}} \| p_{Y \mid X} \mid p_{\boldsymbol{x}}\right)} \\
= & 2^{-N E_{s p, N}\left(\nu, p_{\boldsymbol{x}}, p_{Y \mid X}\right)} .
\end{aligned}
$$

Incorrect Messages: The codewords are drawn independently and uniformly from type class $T_{\boldsymbol{x}}$. Since the conditional error probability is independent of the transmitted message, assume without loss of generality that message $m=1$ was transmitted. An incorrect codeword $\boldsymbol{x}(i)$ appears on the decoder's list if $i>1$ and

$$
I(\boldsymbol{x}(i) ; \boldsymbol{y}) \geq R+\max _{j \neq i} F(I(\boldsymbol{x}(j) ; \boldsymbol{y})-R) .
$$

Let $\boldsymbol{x}=\boldsymbol{x}(1)$. To evaluate the expected number of incorrect codewords on the list, we first fix $\boldsymbol{y}$.

Given $\boldsymbol{y}$, define the i.i.d. random variables $Z_{i}=I(\boldsymbol{x}(i) ; \boldsymbol{y})-$ $R$ for $2 \leq i \leq 2^{N R}$. Also, let $z_{1}=I(\boldsymbol{x} ; \boldsymbol{y})-R$, which is a function of the joint type $p_{x y}$. The expected number of incorrect codewords on the list depends on $(\boldsymbol{x}, \boldsymbol{y})$ only via their joint type and is given by

$$
\begin{aligned}
\mathbb{E}\left[N_{i} \mid T_{\boldsymbol{x y}}\right]= & \sum_{i=2}^{2^{N R} \operatorname{Pr}}[I(\boldsymbol{x}(i) ; \boldsymbol{y}) \geq R \\
& \left.\quad+\max _{j \notin \mathcal{M} \backslash\{i\}} F(I(\boldsymbol{x}(j) ; \boldsymbol{y})-R)\right] \\
= & \sum_{i=2}^{2^{N R} \operatorname{Pr}}\left[Z_{i} \geq \max _{j \notin \mathcal{M} \backslash\{i\}} F\left(Z_{j}\right)\right] \\
\leq & \sum_{i=2}^{2^{N R}} \operatorname{Pr}\left[Z_{i} \geq F\left(z_{1}\right)\right] \\
= & \left(2^{N R}-1\right) \operatorname{Pr}\left[Z_{2} \geq F\left(z_{1}\right)\right]
\end{aligned}
$$

$$
\begin{aligned}
& \stackrel{(\mathrm{a})}{=}\left(2^{N R}-1\right) \\
& \quad \times \operatorname{Pr}\left[I\left(\boldsymbol{x}^{\prime} ; \boldsymbol{y}\right) \geq R+F(I(\boldsymbol{x} ; \boldsymbol{y})-R)\right] \\
& \stackrel{(\mathrm{b})}{\doteq} 2^{N R} 2^{-N[R+F(I(\boldsymbol{x} ; \boldsymbol{y})-R)]} \\
& =2^{-N F(I(\boldsymbol{x} ; \boldsymbol{y})-R)}
\end{aligned}
$$

where in (a), $\boldsymbol{x}^{\prime}$ is drawn independently of $\boldsymbol{y}$ and uniformly over the type class $T_{x}$; and (b) is obtained by application of (B.4).

Averaging over $\boldsymbol{y}$, we obtain

$$
\begin{aligned}
\mathbb{E}\left[N_{i} \mid T_{\boldsymbol{x}}\right] & =\sum_{T_{\boldsymbol{y} \mid \boldsymbol{x}}} \operatorname{Pr}\left[T_{\boldsymbol{y} \mid \boldsymbol{x}}\right] \mathbb{E}\left[N_{i} \mid T_{\boldsymbol{x} \boldsymbol{y}}\right] \\
& \doteq \max _{T_{\boldsymbol{y} \mid \boldsymbol{x}}} \operatorname{Pr}\left[T_{\boldsymbol{y} \mid \boldsymbol{x}}\right] \mathbb{E}\left[N_{i} \mid T_{\boldsymbol{x} \boldsymbol{y}}\right] \\
& \stackrel{(\mathrm{a})}{\leq} \max _{p_{\boldsymbol{y} \mid \boldsymbol{x}}} \exp _{2}\left\{-N\left[D\left(p_{\boldsymbol{y} \mid \boldsymbol{x}} \| p_{Y \mid X} \mid p_{\boldsymbol{x}}\right)\right.\right. \\
& \quad+F(I(\boldsymbol{x} ; \boldsymbol{y})-R)]\} \\
& \stackrel{\text { (b) }}{=} \exp _{2}\left\{-N E_{r, F, N}\left(R, p_{\boldsymbol{x}}, p_{Y \mid X}\right)\right\} \\
& \stackrel{\text { (c) }}{=} \exp _{2}\left\{-N E_{r, F}\left(R, p_{X}, p_{Y \mid X}\right)\right\}
\end{aligned}
$$

where (a) follows from (B.5) and (B.7), (b) from (B.1), and (c) from (B.3). This proves (4.8).

Erasure: The decoder fails to return the transmitted codeword $\boldsymbol{x}=\boldsymbol{x}(1)$ if

$$
I(\boldsymbol{x} ; \boldsymbol{y}) \leq R+\max _{2 \leq i \leq 2^{N R}} F(I(\boldsymbol{x}(i) ; \boldsymbol{y})-R) .
$$

Denote by $p_{\emptyset}\left(p_{\boldsymbol{x}}\right)$ the probability of this event. The event is the disjoint union of events $\mathcal{E}_{1}$ and $\mathcal{E}_{2}$ below. The first one is

$$
\mathcal{E}_{1}: I(\boldsymbol{x} ; \boldsymbol{y}) \leq R+F(0) .
$$

Since $F^{-1}$ is increasing, $\mathcal{E}_{1}$ is equivalent to $F^{-1}(I(\boldsymbol{x} ; \boldsymbol{y})-R) \leq$ 0 . The second event is

$$
\begin{aligned}
\mathcal{E}_{2} & : R+F(0)<I(\boldsymbol{x} ; \boldsymbol{y}) \\
& \leq R+\max _{2 \leq i \leq 2^{N R}} F(I(\boldsymbol{x}(i) ; \boldsymbol{y})-R) \\
& =R+F\left(\max _{2 \leq i \leq 2^{N R}} I(\boldsymbol{x}(i) ; \boldsymbol{y})-R\right)
\end{aligned}
$$

where equality holds because $F$ is nondecreasing. Thus, $\mathcal{E}_{2}$ is equivalent to

$$
\begin{aligned}
\max _{2 \leq i \leq 2^{N R}} I(\boldsymbol{x}(i) ; \boldsymbol{y}) & \geq R+F^{-1}(I(\boldsymbol{x} ; \boldsymbol{y})-R) \\
& >R
\end{aligned}
$$

where the last inequality follows from the fact that $I(\boldsymbol{x} ; \boldsymbol{y})-R>$ $F(0)$ under $\mathcal{E}_{2}$.

Applying (B.6) and (B.3) successively, we have

$$
\begin{aligned}
\operatorname{Pr}\left[\mathcal{E}_{1}\right] & =p_{\emptyset}^{(1)}\left(p_{\boldsymbol{x}}\right) \\
& \triangleq \operatorname{Pr}[I(\boldsymbol{x} ; \boldsymbol{y}) \leq R+F(0)] \\
& \doteq \exp _{2}\left\{-N E_{s p, N}\left(R+F(0), p_{\boldsymbol{x}}, p_{Y \mid X}\right)\right\} \\
& \doteq \exp _{2}\left\{-N E_{\mathrm{sp}}\left(R+F(0), p_{\boldsymbol{x}}, p_{Y \mid X}\right)\right\} .
\end{aligned}
$$


Clearly $p_{\emptyset}\left(p_{\boldsymbol{x}}\right) \sim p_{\emptyset}^{(1)}\left(p_{\boldsymbol{x}}\right) \sim 1$ if

$$
R+F(0) \geq I\left(p_{\boldsymbol{x}}, p_{Y \mid X}\right) .
$$

Next, the probability of $\mathcal{E}_{2}$ conditioned on $\boldsymbol{y}$ is

$$
\begin{aligned}
p_{\emptyset}^{(2)}\left(T_{\boldsymbol{y} \mid \boldsymbol{x}}\right)= & \operatorname{Pr}\left[\max _{2 \leq i \leq 2^{N R}} I(\boldsymbol{x}(i) ; \boldsymbol{y}) \geq R\right. \\
& \left.\quad+F^{-1}(I(\boldsymbol{x} ; \boldsymbol{y})-R)\right] \\
& \stackrel{(\mathrm{a})}{\leq} 2^{N R} \operatorname{Pr}\left[I\left(\boldsymbol{x}^{\prime} ; \boldsymbol{y}\right) \geq R+F^{-1}(I(\boldsymbol{x} ; \boldsymbol{y})-R)\right] \\
& \stackrel{(\mathrm{b})}{\doteq} 2^{-N F^{-1}(I(\boldsymbol{x} ; \boldsymbol{y})-R)}
\end{aligned}
$$

where (a) follows from the union bound, and $\boldsymbol{x}^{\prime}$ is drawn independently of $\boldsymbol{x}$ and $\boldsymbol{y}$ and uniformly from the type class $T_{\boldsymbol{x}}$; and (b) follows from (B.4).

Averaging over $y$, we obtain (B.13) at the bottom of the page, where (a) follows from (B.5) and (B.12) and (b) from the same continuity argument that was used to establish (B.2). Note that $F^{-1}\left(I\left(p_{\boldsymbol{x}}, \tilde{p}_{Y \mid X}\right)-R\right)=0$ for $I\left(p_{\boldsymbol{x}}, \tilde{p}_{Y \mid X}\right)=R+F(0)$. In view of the comment following (B.11), we need to bound $p_{\emptyset}^{(2)}\left(p_{\boldsymbol{x}}\right)$ only in the nontrivial case $R+F(0) \leq I\left(p_{\boldsymbol{x}}, p_{Y \mid X}\right)$. Then, analogously to (A.2), we have

$\max _{\tilde{p}_{Y \mid X}: I\left(p_{\boldsymbol{x}}, \tilde{p}_{Y \mid X}\right) \leq R+F(0)} \exp _{2}\left\{-N D\left(\tilde{p}_{Y \mid X} \| p_{Y \mid X} \mid p_{\boldsymbol{x}}\right)+0\right\}$

$=\exp _{2}\left\{-N E_{\mathrm{sp}}\left(R+F(0), p_{\boldsymbol{x}}, p_{Y \mid X}\right)\right\}$

$=\max _{\tilde{p}_{Y \mid X}: I\left(p_{\boldsymbol{x}}, \tilde{p}_{Y \mid X}\right)=R+F(0)} \exp _{2}\left\{-N D\left(\tilde{p}_{Y \mid X} \| p_{Y \mid X} \mid p_{\boldsymbol{x}}\right)+0\right\}$.

Thus, (B.13) reduces to the unconstrained maximum

$$
\begin{aligned}
& p_{\emptyset}^{(2)}\left(p_{\boldsymbol{x}}\right) \doteq \max _{\tilde{p}_{Y \mid X}} \exp _{2}\left\{-N\left[D\left(\tilde{p}_{Y \mid X} \| p_{Y \mid X} \mid p_{\boldsymbol{x}}\right)\right.\right. \\
&\left.\left.\quad+\left|F^{-1}\left(I\left(p_{\boldsymbol{x}}, \tilde{p}_{Y \mid X}\right)-R\right)\right|^{+}\right]\right\} \\
&=\exp _{2}\left\{-N E_{r,\left|F^{-1}\right|^{+}}\left(R, p_{\boldsymbol{x}}, p_{Y \mid X}\right)\right\} .
\end{aligned}
$$

Since $\mathcal{E}_{1}$ and $\mathcal{E}_{2}$ are disjoint events, we obtain

$$
\begin{gathered}
p_{\emptyset}\left(p_{\boldsymbol{x}}\right)=p_{\emptyset}^{(1)}\left(p_{\boldsymbol{x}}\right)+p_{\emptyset}^{(2)}\left(p_{\boldsymbol{x}}\right) \\
\doteq \exp _{2}\left\{-N \min \left\{E_{\mathrm{sp}}\left(R+F(0), p_{X}, p_{Y \mid X}\right),\right.\right. \\
\left.\left.E_{r,\left|F^{-1}\right|^{+}}\left(R, p_{X}, p_{Y \mid X}\right)\right\}\right\} .
\end{gathered}
$$

The function $F^{-1}(t)$ has a zero at $t=F(0)$. Applying (4.7), we obtain

$$
\begin{aligned}
& E_{r,\left|F^{-1}\right|^{+}}\left(R, p_{X}, p_{Y \mid X}\right) \\
& \quad=\min _{R^{\prime}}\left[E_{\mathrm{sp}}\left(R^{\prime}, p_{X}, p_{Y \mid X}\right)+\left|F^{-1}\left(R^{\prime}-R\right)\right|^{+}\right] \\
& \quad \leq E_{\mathrm{sp}}\left(R+F(0), p_{X}, p_{Y \mid X}\right)+0 .
\end{aligned}
$$

Hence

$$
p_{\emptyset}\left(p_{\boldsymbol{x}}\right) \dot{\leq} \exp _{2}\left\{-N E_{r,\left|F^{-1}\right|^{+}}\left(R, p_{X}, p_{Y \mid X}\right)\right\},
$$

which proves (4.9).

\section{APPENDIX C \\ PROOF OF PROPOSITION 5.1}

We first prove (5.11). Recall (4.7) and (4.6), restated here for convenience:

$$
\begin{aligned}
E_{r, F}\left(R, p_{X}, \mathcal{W}\right) & =\min _{R^{\prime}}\left[E_{\mathrm{sp}}\left(R^{\prime}, p_{X}, \mathcal{W}\right)+F\left(R^{\prime}-R\right)\right] \\
E_{\mathrm{sp}}\left(R, p_{X}, \mathcal{W}\right) & \equiv E_{\mathrm{sp}}\left(R^{\prime}, p_{X}, \mathcal{W}\right)+F_{R, p_{X}, \mathcal{W}}\left(R^{\prime}-R\right),
\end{aligned}
$$

$\forall R^{\prime}$.

\section{Hence}

$$
E_{r, F_{R, p_{X}, \mathcal{W}}}\left(R, p_{X}, \mathcal{W}\right)=E_{\mathrm{sp}}\left(R, p_{X}, \mathcal{W}\right) .
$$

We have

$$
\begin{aligned}
\alpha & \stackrel{(\mathrm{a})}{=} \Delta+E_{\mathrm{sp}}\left(R, p_{X}, \mathcal{W}\right) \\
& \stackrel{(\mathrm{b})}{=} \Delta+E_{r, F_{R, p_{X}, \mathcal{W}}}\left(R, p_{X}, \mathcal{W}\right) \\
& \stackrel{(\mathrm{c})}{=} E_{r, \Delta+F_{R, p_{X}, \mathcal{W}}}\left(R, p_{X}, \mathcal{W}\right) \\
& \stackrel{(\mathrm{d})}{=} E_{F^{L *}}\left(R, p_{X}, \mathcal{W}\right)
\end{aligned}
$$

where (a) follows from (5.9) (b) from (C.1), (c) from the definition (4.3) of $E_{r, F}(\cdot)$, and (d) from the definition (5.11) of $F^{L *}$. The feasible set $\mathcal{F}^{L}\left(R, p_{X}, \mathcal{W}, \alpha\right)$ defined in (5.5) takes the form

$$
\begin{aligned}
\mathcal{F}^{L}\left(R, p_{X}, \mathcal{W}, \alpha\right)= & \left\{F: E_{r, F}\left(R, p_{X}, \mathcal{W}\right) \geq \alpha\right\} \\
= & \left\{F: E_{r, F}\left(R, p_{X}, \mathcal{W}\right)\right. \\
& \left.\geq E_{r, F^{L *}}\left(R, p_{X}, \mathcal{W}\right)\right\} \\
= & \left\{F: F \succeq F^{L *}\right\}
\end{aligned}
$$

$$
\begin{aligned}
\operatorname{Pr}\left[\mathcal{E}_{2}\right]=p_{\emptyset}^{(2)}\left(p_{\boldsymbol{x}}\right) & \triangleq \sum_{T_{\boldsymbol{y} \mid \boldsymbol{x}}: I(\boldsymbol{x} ; \boldsymbol{y})>R+F(0)} \operatorname{Pr}\left[T_{\boldsymbol{y} \mid \boldsymbol{x}}\right] p_{\emptyset}^{(2)}\left(T_{\boldsymbol{y} \mid \boldsymbol{x}}\right) \\
& \doteq \max _{T_{\boldsymbol{y} \mid \boldsymbol{x}}: I(\boldsymbol{x} ; \boldsymbol{y}) \geq R+F(0)} \operatorname{Pr}\left[T_{\boldsymbol{y} \mid \boldsymbol{x}}\right] p_{\emptyset}^{(2)}\left(T_{\boldsymbol{y} \mid \boldsymbol{x}}\right) \\
& \stackrel{(\mathrm{a})}{\doteq} \max _{p_{\boldsymbol{y} \mid \boldsymbol{x}}: I(\boldsymbol{x} ; \boldsymbol{y}) \geq R+F(0)} \exp _{2}\left\{-N\left[D\left(p_{\boldsymbol{y} \mid \boldsymbol{x}} \| p_{Y \mid X} \mid p_{\boldsymbol{x}}\right)+F^{-1}(I(\boldsymbol{x} ; \boldsymbol{y})-R)\right]\right\} \\
& \stackrel{(\mathrm{b})}{\doteq} \operatorname{pr}_{Y \mid X} \max _{\tilde{p}_{Y}: I\left(p_{\boldsymbol{x}}, \tilde{r}_{Y \mid X}\right) \geq R+F(0)} \exp _{2}\left\{-N\left[D\left(\tilde{p}_{Y \mid X} \| p_{Y \mid X} \mid p_{\boldsymbol{x}}\right)+F^{-1}\left(I\left(p_{\boldsymbol{x}}, \tilde{p}_{Y \mid X}\right)-R\right)\right]\right\}
\end{aligned}
$$


where the last line follows from the monotonicity property of Proposition 4.1(ii). As indicated below the statement of Proposition 5.1, this implies $F^{L *}$ achieves the supremum in (5.5).

The alternative expression (5.12) for $F^{L *}$ is obtained by substituting (4.5) and (5.9) into (5.11).

To prove (5.13), we simply observe that if $F^{L *} \preceq F$ for all $F \in \mathcal{F}^{L}\left(R, p_{X}, \mathcal{W}, \alpha\right)$, then $F^{*} \preceq F$ for all $F \in$ $\mathcal{F}\left(R, p_{X}, \mathcal{W}, \alpha\right)$, where $F^{*}(t)=\max \left(t, F^{L *}(t)\right)$.

\section{APPENDIX D}

\section{PROOF OF PROPOSITION 5.3}

We have

$$
\begin{aligned}
& E_{\emptyset}^{L}\left(R, p_{X}, \mathcal{W}, \alpha\right) \stackrel{(\mathrm{a})}{=} E_{r,\left|\left(F^{L *}\right)^{-1}\right|^{+}}\left(R, p_{X}, \mathcal{W}\right) \\
& \stackrel{(\mathrm{b})}{=} \min _{R^{\prime}}\left[E_{\mathrm{sp}}\left(R^{\prime}, p_{X}, \mathcal{W}\right)\right. \\
&\left.\quad+\left|\left(F^{L *}\right)^{-1}\right|^{+}\left(R^{\prime}-R\right)\right]
\end{aligned}
$$

where equality (a) results from Propositions 4.2 and 5.1, and (b) from (4.5). From (5.11) and property (P3) in Section III, we obtain the inverse function

$$
\left(F^{L *}\right)^{-1}(t)=F_{R, p_{X}, \mathcal{W}}^{-1}(t-\Delta)
$$

where $F_{R, p_{X}, \mathcal{W}}(t)$ is given in (4.5). We have $\left(F^{L *}\right)^{-1}(0)=0$ and thus $\left|\left(F^{L *}\right)^{-1}(t)\right|^{+}=0$ for all $t \leq 0$. Since the function $E_{\mathrm{sp}}\left(R^{\prime}, p_{X}, \mathcal{W}\right)$ is nonincreasing in $R^{\prime}$, we may write (D.1) as

$$
\begin{aligned}
& E_{\emptyset}^{L}\left(R, p_{X}, \mathcal{W}, \alpha\right)= \\
& \min _{R^{\prime} \geq R+\Delta}[\underbrace{E_{\mathrm{sp}}\left(R^{\prime}, p_{X}, \mathcal{W}\right)+F_{R, p_{X}, \mathcal{W}}^{-1}\left(R^{\prime}-R-\Delta\right)}_{h\left(R^{\prime}\right)}] . \quad \text { (D.3) }
\end{aligned}
$$

By assumption, $E_{\mathrm{sp}}\left(R, p_{X}, \mathcal{W}\right)$ is convex in $R$, and therefore $F_{R, p_{X}, \mathcal{W}}(t)$ is concave. By application of Property (P5) in Section III, the function $F_{R, p_{X}, \mathcal{W}}^{-1}$ is convex, and thus so is $h\left(R^{\prime}\right)$ in (D.3). The derivative of $F_{R, p_{X}, \mathcal{W}}^{-1}$ is given by

$$
\begin{aligned}
\left(F_{R, p_{X}, \mathcal{W}}^{-1}\right)^{\prime}(t) & \stackrel{(\mathrm{a})}{=} \frac{1}{F_{R, p_{X}, \mathcal{W}}^{\prime}(t)} \\
& \stackrel{(\mathrm{b})}{=} \frac{1}{-E_{\mathrm{sp}}^{\prime}\left(R+t, p_{X}, \mathcal{W}\right)}
\end{aligned}
$$

where (a) follows from Property (P4) and (b) from (4.6). Differentiating $h\left(R^{\prime}\right)$ defined in (D.3) and using (D.4) we obtain

$$
h^{\prime}\left(R^{\prime}\right)=E_{\mathrm{sp}}^{\prime}\left(R^{\prime}, p_{X}, \mathcal{W}\right)+\frac{1}{-E_{\mathrm{sp}}^{\prime}\left(R^{\prime}-\Delta, p_{X}, \mathcal{W}\right)} .
$$

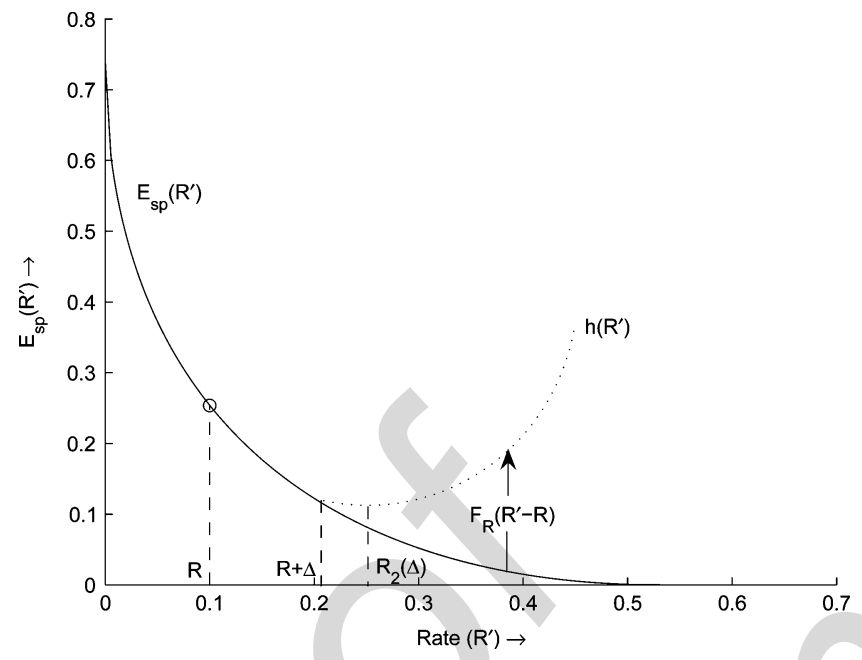

Fig. 3. Construction and minimization of $h\left(R^{\prime}\right)$ for Case (i).

By Proposition 5.1 and the definition of $\Delta$ in (5.9), we have

$$
E_{i}\left(R, p_{X}, \mathcal{W}\right)=\alpha=E_{\mathrm{sp}}\left(R, p_{X}, \mathcal{W}\right)+\Delta
$$

Next we prove the statements (i)-(iv).

(i) It is assumed that

$$
\max \left(0, R^{\operatorname{conj}}\left(p_{X}, \mathcal{W}\right)-R\right) \leq \Delta \leq I\left(p_{X}, \mathcal{W}\right)-R .
$$

This case is illustrated in Fig. 3. We have

$$
R+\Delta \geq \max \left(R, R^{\operatorname{conj}}\left(p_{X}, \mathcal{W}\right)\right) \geq R_{c r}\left(p_{X}, \mathcal{W}\right) .
$$

Hence from (D.5) we have (D.7) shown at the bottom of the page. By convexity of $h(\cdot)$, this implies that $R+\Delta$ minimizes $h\left(R^{\prime}\right)$ over $R^{\prime} \geq R+\Delta$, and so

$$
E_{\emptyset}^{L}\left(R, p_{X}, \mathcal{W}, \alpha\right)=h(R+\Delta)=E_{\mathrm{sp}}\left(R+\Delta, p_{X}, \mathcal{W}\right) .
$$

(ii) Due to (D.6), we have either $R \geq R_{c r}\left(p_{X}, \mathcal{W}\right)$ or $R+\Delta \geq R^{\text {conj }}\left(p_{X}, \mathcal{W}\right) \geq R$. In both cases

$$
-E_{\mathrm{sp}}^{\prime}\left(R, p_{X}, \mathcal{W}\right) \leq \frac{1}{-E_{\mathrm{sp}}^{\prime}\left(R+\Delta, p_{X}, \mathcal{W}\right)} .
$$

Let $F(t)=\Delta+\lambda|t|^{+}$where $\lambda$ is sandwiched by the left and right sides of the above inequality. We have $F^{\prime}(t)=\lambda \mathbb{1}_{\{t \geq 0\}}$. The inverse function is $F^{-1}(t)=\frac{1}{\lambda}(t-\Delta)$ for $t \geq \Delta$. Hence

$$
\begin{aligned}
h^{\prime}(R+\Delta) & =E_{\mathrm{sp}}^{\prime}\left(R+\Delta, p_{X}, \mathcal{W}\right)+\frac{1}{-E_{\mathrm{sp}}^{\prime}\left(R, p_{X}, \mathcal{W}\right)} \\
& \geq \begin{cases}E_{\mathrm{sp}}^{\prime}\left(R, p_{X}, \mathcal{W}\right)+\frac{1}{-E_{\mathrm{sp}}^{\prime}\left(R, p_{X}, \mathcal{W}\right)} \geq 0 & : \text { if } R \geq R_{c r}\left(p_{X}, \mathcal{W}\right) \\
E_{\mathrm{sp}}^{\prime}\left(R^{\operatorname{conj}}\left(p_{X}, \mathcal{W}\right), p_{X}, \mathcal{W}\right)+\frac{1}{-E_{\mathrm{sp}}^{\prime}\left(R, p_{X}, \mathcal{W}\right)}=0 & : \text { if } R \leq R_{c r}\left(p_{X}, \mathcal{W}\right)\end{cases} \\
& \geq 0 .
\end{aligned}
$$




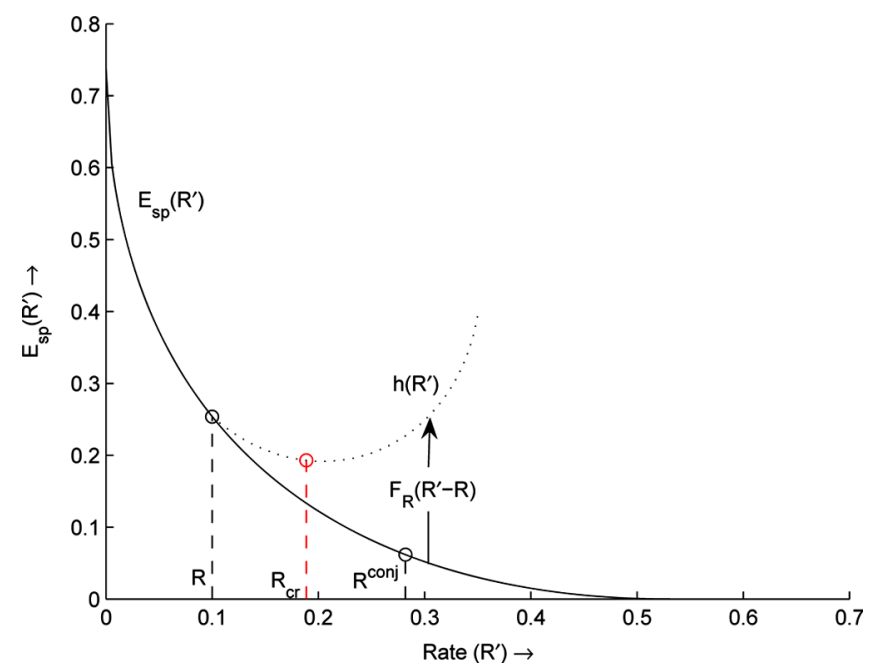

Fig. 4. Construction and minimization of $h\left(R^{\prime}\right)$ for Case (ii), with $\Delta=0$.

$\left(F^{-1}\right)^{\prime}(t)=\frac{1}{\lambda}$ for $t \geq \Delta$. Substituting $F$ and $F^{-1}$ into (4.7), we obtain

$$
\begin{aligned}
& E_{r, F}\left(R, p_{X}, \mathcal{W}\right) \\
& \quad=\min _{R^{\prime} \geq R}\left[E_{\mathrm{sp}}\left(R^{\prime}, p_{X}, \mathcal{W}\right)+\Delta+\lambda\left(R^{\prime}-R\right)\right] \\
& E_{r,\left|F^{-1}\right|^{+}}\left(R, p_{X}, \mathcal{W}\right) \\
& \quad=\min _{R^{\prime} \geq R+\Delta}\left[E_{\mathrm{sp}}\left(R^{\prime}, p_{X}, \mathcal{W}\right)+\frac{1}{\lambda}\left(R^{\prime}-R-\Delta\right)\right] .
\end{aligned}
$$

Taking derivatives of the bracketed terms with respect to $R^{\prime}$ and recalling that

$$
\lambda \geq-E_{\mathrm{sp}}^{\prime}\left(R, p_{X}, \mathcal{W}\right), \quad \frac{1}{\lambda} \geq-E_{\mathrm{sp}}^{\prime}\left(R+\Delta, p_{X}, \mathcal{W}\right)
$$

we observe that these derivatives are nonnegative. Since $E_{\mathrm{sp}}\left(\cdot, p_{X}, \mathcal{W}\right)$ is convex, the minima are achieved at $R$ and $R+\Delta$, respectively.

The resulting exponents are $E_{\mathrm{sp}}\left(R, p_{X}, \mathcal{W}\right)+\Delta$ and $E_{\mathrm{sp}}\left(R+\Delta, p_{X}, \mathcal{W}\right)$ which coincide with the optimal exponents of (5.14).

(iii) $R \leq R_{c r}\left(p_{X}, \mathcal{W}\right)$ and $0 \leq \Delta \leq R^{\operatorname{conj}}\left(p_{X}, \mathcal{W}\right)-R$. This case is illustrated in Fig. 4 in the case $\Delta=0$. From (D.7), we have $h^{\prime}\left(R^{\prime}\right)=0$ if and only if $R^{\prime}$ and $R^{\prime}-\Delta$ are conjugate rates. In this case, using the above assumption on $\Delta$, we have

$$
\begin{aligned}
R \leq R^{\prime}-\Delta & =R_{1}(\Delta) \leq R_{c r}\left(p_{X}, \mathcal{W}\right) \leq R^{\prime} \\
& =R_{2}(\Delta) \leq R^{\operatorname{conj}}\left(p_{X}, \mathcal{W}\right)
\end{aligned}
$$

Hence, $R_{2}(\Delta)=R^{\prime} \geq R+\Delta$ is feasible for (D.3) and minimizes $h(\cdot)$. Substituting $R^{\prime}$ back into (D.3), we obtain

$$
\begin{aligned}
E_{\emptyset}^{L}\left(R, p_{X}, \mathcal{W}, \alpha\right)= & h\left(R_{2}(\Delta)\right) \\
= & E_{\mathrm{sp}}\left(R_{2}(\Delta), p_{X}, \mathcal{W}\right) \\
& +F_{R, p_{X}, \mathcal{W}}^{-1}\left(R_{1}(\Delta)-R\right)
\end{aligned}
$$

which establishes (5.16).

(iv) $R \leq R_{c r}\left(p_{X}, \mathcal{W}\right)$ and $R_{\infty}\left(p_{X}, \mathcal{W}\right)-R \leq \Delta \leq 0$.
Again we have $h^{\prime}\left(R^{\prime}\right)=0$ if and only if $R^{\prime}$ and $R^{\prime}-\Delta$ are conjugate rates. Then, using the above assumption on $\Delta$, we have

$$
R \leq R^{\prime}=R_{1}(\Delta) \leq R_{c r}\left(p_{X}, \mathcal{W}\right) \leq R^{\prime}-\Delta=R_{2}(\Delta)
$$

Hence $R_{1}(\Delta)=R^{\prime} \geq R+\Delta$ is feasible for (D.3) and minimizes $h(\cdot)$. Substituting $R^{\prime}$ back into (D.3), we obtain

$$
\begin{aligned}
E_{\emptyset}^{L}\left(R, p_{X}, \mathcal{W} \alpha\right)= & h\left(R_{1}(\Delta)\right) \\
= & E_{\mathrm{sp}}\left(R_{1}(\Delta), p_{X}, \mathcal{W}\right) \\
& +F_{R, p_{X}, \mathcal{W}}^{-1}\left(R_{2}(\Delta)-R\right)
\end{aligned}
$$

which establishes (5.17).

\section{APPENDIX E}

PROOF OF LEMMA 5.4

First we prove (5.20). For any $R_{0}<R_{1}$, we have

$$
\begin{aligned}
\int_{R_{0}}^{R_{1}} & \underline{E_{\mathrm{sp}}^{\prime}}\left(R, p_{X}, \mathcal{W}\right) d R \\
& \stackrel{(\mathrm{a})}{=} \int_{R_{0}}^{R_{1}} \min _{p_{Y \mid X} \in \mathcal{W}} E_{\mathrm{sp}}^{\prime}\left(R, p_{X}, p_{Y \mid X}\right) d R \\
& \leq \min _{p_{Y \mid X} \in \mathcal{W}} \int_{R_{0}}^{R_{1}} E_{\mathrm{sp}}^{\prime}\left(R, p_{X}, p_{Y \mid X}\right) d R \\
& =\min _{p_{Y \mid X} \in \mathcal{W}}\left[E_{\mathrm{sp}}\left(R_{1}, p_{X}, p_{Y \mid X}\right)-E_{\mathrm{sp}}\left(R_{0}, p_{X}, p_{Y \mid X}\right)\right] \\
& \stackrel{\mathrm{b})}{\leq} E_{\mathrm{sp}}\left(R_{1}, p_{X}, p_{Y \mid X}^{*}\right)-E_{\mathrm{sp}}\left(R_{0}, p_{X}, p_{Y \mid X}^{*}\right) \\
& =\min _{p_{Y \mid X} \in \mathcal{W}} E_{\mathrm{sp}}\left(R_{1}, p_{X}, p_{Y \mid X}\right)-E_{\mathrm{sp}}\left(R_{0}, p_{X}, p_{Y \mid X}^{*}\right) \\
& \leq \min _{p_{Y \mid X} \in \mathcal{W}} E_{\mathrm{sp}}\left(R_{1}, p_{X}, p_{Y \mid X}\right)-\min _{p_{Y \mid X} \in \mathcal{W}} E_{\mathrm{sp}}\left(R_{0}, p_{X}, p_{Y \mid X}\right) \\
& =E_{\mathrm{sp}}\left(R_{1}, p_{X}, \mathcal{W}\right)-E_{\mathrm{sp}}\left(R_{0}, p_{X}, \mathcal{W}\right) \\
& =\int_{R_{0}}^{R_{1}} E_{\mathrm{sp}}^{\prime}\left(R, p_{X}, \mathcal{W}\right) d R
\end{aligned}
$$

where (a) follows from the definition of $\underline{E}_{\mathrm{sp}}^{\prime}$ in (5.18), and we choose $p_{Y \mid X}^{*}$ in inequality (b) as the minimizer of $E_{\mathrm{sp}}\left(R_{1}, p_{X}, \cdot\right)$ over $\mathcal{W}$. Since (E.1) holds for all $R_{0}<R_{1}$, we must have inequality between the integrands in the left and right sides: $\underline{E}_{\mathrm{sp}}^{\prime}\left(R, p_{X}, \mathcal{W}\right) \leq E_{\mathrm{sp}}^{\prime}\left(R, p_{X}, \mathcal{W}\right)$. Moreover, the three inequalities used to derive (E.1) hold with equality if the same $p_{Y \mid X}^{* *}$ minimizes $E_{\mathrm{sp}}^{\prime}\left(R, p_{X}, \cdot\right)$ at all rates, and the same $p_{Y \mid X}^{*}$ minimizes $E_{\mathrm{sp}}\left(R, p_{X}, \cdot\right)$ at all rates. We need not (and generally do not) have $p_{Y \mid X}^{* *}=p_{Y \mid X}^{*} \cdot{ }^{5}$

Next we prove (5.21). By definition of $R^{\operatorname{conj}}\left(p_{X}, \mathcal{W}\right)$, we have

$$
\begin{gathered}
-E_{\mathrm{sp}}^{\prime}\left(R^{\mathrm{conj}}\left(p_{X}, \mathcal{W}\right), p_{X}, \mathcal{W}\right) \\
=\frac{1}{-E_{\mathrm{sp}}^{\prime}\left(R, p_{X}, \mathcal{W}\right)} \\
\quad \stackrel{(\mathrm{a})}{\geq} \frac{1}{-\underline{E}_{\mathrm{sp}}^{\prime}\left(R, p_{X}, \mathcal{W}\right)}
\end{gathered}
$$

${ }^{5}$ While $p_{Y \mid X}^{*}$ is the noisiest channel in $\mathcal{W}, p_{Y \mid X}^{* *}$ may be the cleanest channel in $\mathcal{W}$, as in the BSC example of Section VII 


$$
\begin{aligned}
& =\min _{p_{Y \mid X} \in \mathcal{W}} \frac{1}{-E_{\mathrm{sp}}^{\prime}\left(R, p_{X}, p_{Y \mid X}\right)} \\
& =\min _{p_{Y \mid X} \in \mathcal{W}}\left[-E_{\mathrm{sp}}^{\prime}\left(R^{\mathrm{conj}}\left(p_{X}, p_{Y \mid X}\right), p_{X}, p_{Y \mid X}\right)\right] \\
& \stackrel{(\mathrm{b})}{\geq} \min _{p_{Y \mid X} \in \mathcal{W}}\left[-E_{\mathrm{sp}}^{\prime}\left(\bar{R}^{\mathrm{conj}}\left(p_{X}, \mathcal{W}\right), p_{X}, p_{Y \mid X}\right)\right] \\
& \stackrel{(\mathrm{c})}{=}-\underline{E}_{\mathrm{sp}}^{\prime}\left(\bar{R}^{\mathrm{conj}}\left(p_{X}, \mathcal{W}\right), p_{X}, \mathcal{W}\right) \\
& \stackrel{(\mathrm{d})}{\geq}-E_{\mathrm{sp}}^{\prime}\left(\bar{R}^{\mathrm{conj}}\left(p_{X}, \mathcal{W}\right), p_{X}, \mathcal{W}\right)
\end{aligned}
$$

where (a) and (d) are due to (5.20), (b) to the definition of $\bar{R}^{\mathrm{conj}}\left(p_{X}, \mathcal{W}\right)$ in (5.19) and the fact that $-E_{\mathrm{sp}}^{\prime}\left(R, p_{X}, p_{Y \mid X}\right)$ is a decreasing function of $R$, and (c) from (5.18). Since $-E_{\mathrm{sp}}^{\prime}\left(R, p_{X}, \mathcal{W}\right)$ is also a decreasing function of $R$, we must have $R^{\text {conj }}\left(p_{X}, \mathcal{W}\right) \leq \bar{R}^{\text {conj }}\left(p_{X}, \mathcal{W}\right)$. Moreover, the conditions for equality are the same as those for equality in (5.20).

\section{ACKNOWLEDGMENT}

The author wishes to thank Shankar Sadasivam for numerical evaluation of the error exponent formulas in Section VII, Prof. Lizhong Zheng for helpful comments, and the reviewers for their careful reading of the manuscript and recommended improvements.

\section{REFERENCES}

[1] I. Csiszár and J. Körner, Information Theory: Coding Theory for Discrete Memoryless Systems. New York: Academic, 1981.

[2] A. Lapidoth and P. Narayan, "Reliable communication under channel uncertainty," IEEE Trans. Inf. Theory, vol. 44, no. 6, pp. 2148-2177, Oct. 1998.

[3] G. D. Forney, Jr, "Exponential error bounds for erasure, list, and decision feedback schemes," IEEE Trans. Inf. Theory, vol. IT-14, no. 2, pp. 206-220, Mar. 1968.

[4] I. E. Telatar, "Multi-Access Communications With Decision Feedback Decoding," Ph.D. dissertation, MIT, Cambridge, MA, 1992.
[5] I. E. Telatar and R. G. Gallager, "New exponential upper bounds to error and erasure probabilities," in Proc. IEEE Int. Symp. Information Theory (ISIT'94), Trondheim, Norway, Jun. 1994, p. 379.

[6] N. Merhav and M. Feder, "Minimax universal decoding with erasure option," IEEE Trans. Inf. Theory, vol. 53, no. 5, pp. 1664-1675, May 2007.

[7] S. A. Kassam and H. V. Poor, "Robust techniques for signal processing: A survey," Proc. IEEE, vol. 73, no. 3, pp. 433-481, Mar. 1985.

[8] W. Hoeffding, "Asymptotically optimal tests for multinomial distributions," Ann. Math. Statist., vol. 36, no. 2, pp. 369-400, 1965.

[9] G. Tusnády, "On asymptotically optimal tests," Ann. Statist., vol. 5, no. 2, pp. 385-393, 1977.

[10] O. Zeitouni and M. Gutman, "On universal hypotheses testing via large deviations," IEEE Trans. Inf. Theory, vol. 37, no. 2, pp. 285-290, Mar. 1991.

[11] I. Csiszár, Personal Communication Aug. 2007.

[12] M. Feder and N. Merhav, "Universal composite hypothesis testing: A competitive minimax approach," IEEE Trans. Inf. Theory, vol. 48, no. 6, pp. 1504-1517, Jun. 2002.

Pierre Moulin (S'89-M'90-SM'98-F'03) received the doctoral degree from Washington University, St. Louis, MO, in 1990.

After graduation, he joined at Bell Communications Research, Morristown, NJ, as a Research Scientist. In 1996, he joined the University of Illinois at Urbana-Champaign (UIUC), Urbana, where he is currently Professor in the Department of Electrical and Computer Engineering, Research Professor at the Beckman Institute and the Coordinated Science Laboratory, an affiliate Professor in the Department of Statistics, and Sony Faculty Scholar. His fields of professional interest include image and video processing, compression, statistical signal processing and modeling, media security, decision theory, and information theory.

Dr. Moulin has served on the editorial boards of the IEEE TRANSACTIONS ON INFORMATION THEORY and the IEEE TRANSACTIONS ON IMAGE PROCESSING, He currently serves on the Editorial Boards of PROCEEDINGS OF IEEE and of Foundations and Trends in Signal Processing. He was co-founding Editor-inChief of the IEEE TRANSACTIONS ON INFORMATION FORENSICS AND SECURITY (2005-2008), member of the IEEE Signal Processing Society Board of Governors (2005-2007), and has served IEEE in various other capacities. He received a 1997 CAREER award from the National Science Foundation and an IEEE Signal Processing Society 1997 Senior Best Paper award. He is also coauthor (with Juan Liu) of a paper that received an IEEE Signal Processing Society 2002 Young Author Best Paper award. He was 2003 Beckman Associate of UIUCs Center for Advanced Study and plenary speaker for ICASSP 2006 and several other conferences. 


\title{
A Neyman-Pearson Approach to Universal Erasure and List Decoding
}

\author{
Pierre Moulin, Fellow, IEEE
}

\begin{abstract}
When information is to be transmitted over an unknown, possibly unreliable channel, an erasure option at the decoder is desirable. Using constant-composition random codes, we propose a generalization of Csiszár and Körner's maximum mutual information (MMI) decoder with an erasure option for discrete memoryless channels. The new decoder is parameterized by a weighting function that is designed to optimize the fundamental tradeoff between undetected-error and erasure exponents for a compound class of channels. The class of weighting functions may be further enlarged to optimize a similar tradeoff for list decoders-in that case, undetected-error probability is replaced with average number of incorrect messages in the list. Explicit solutions are identified.

The optimal exponents admit simple expressions in terms of the sphere-packing exponent, at all rates below capacity. For small erasure exponents, these expressions coincide with those derived by Forney (1968) for symmetric channels, using maximum a posteriori decoding. Thus, for those channels at least, ignorance of the channel law is inconsequential. Conditions for optimality of the Csiszár-Körner rule and of the simpler empirical-mutual-information thresholding rule are identified. The error exponents are evaluated numerically for the binary symmetric channel.
\end{abstract}

Index Terms-Constant-composition codes, erasures, error exponents, list decoding, maximum mutual information (MMI) decoder, method of types, Neyman-Pearson hypothesis testing, random codes, sphere packing, universal decoding.

\section{INTRODUCTION}

$\mathbf{U}$ NIVERSAL decoders have been studied extensively in the information theory literature as they are applicable to a variety of communication problems where the channel is partly or even completely unknown [1], [2]. In particular, the maximum mutual information (MMI) decoder provides universally attainable error exponents for random constant-composition codes over discrete memoryless channels (DMCs). In some cases, incomplete knowledge of the channel law is inconsequential as the resulting error exponents are the same as those for

Manuscript received January 29, 2008; revised April 13, 2009. Current version published September 23, 2009. This work was supported by the National Science Foundation (NSF) under Grant CCF 06-35137 and by DARPA under the ITMANET program. The material in this paper was presented in part at the IEEE International Symposium on Information Theory (ISIT), Toronto, ON, Canada, July 2008.

The author is with the Electrical and Computer Engineering Department, Beckman Institute, and the Coordinated Science Laboratory, University of Illinois at Urbana-Champaign, Urbana, IL 61801 USA (e-mail: moulin @ifp.uiuc. edu).

Communicated by H. Yamamoto, Associate Editor for Shannon Theory.

Color versions of Figures 1, 2, and 4 in this paper are available online at http://ieeexplore.ieee.org.

Digital Object Identifier 10.1109/TIT.2009.2027569 maximum-likelihood decoders which know the channel law in effect.

It is often desirable to provide the receiver with an erasure option that can be exercised when the received data are deemed unreliable. For fixed channels, Forney [3] derived the decision rule that provides the optimal tradeoff between the erasure and undetected-error probabilities, analogously to the Neyman-Pearson problem for binary hypothesis testing. Forney used the same framework to optimize the performance of list decoders; the probability of undetected errors is then replaced by the expected number of incorrect messages on the list. The size of the list is a random variable which equals 1 with high probability when communication is reliable. Bounds on the undetected-error and erasure exponents were subsequently derived by Telatar [4], [5] using a decoder parameterized by an asymmetric relation $\prec$ which is a function of the channel law.

For unknown channels, the problem of decoding with erasures was considered by Csiszár and Körner [1]. They derived attainable pairs of undetected-error and erasure exponents for any DMC. However, they did not indicate whether true universality is achievable, i.e., whether the exponents match Forney's exponents. Also they did not indicate whether their error exponents might be optimal in some weaker sense. The problem was recently revisited by Merhav and Feder [6], using a competitive minimax approach. The analysis of [6] yields lower bounds on a certain fraction of the optimal exponents. It is suggested in [6] that true universality might generally not be attainable, which would represent a fundamental difference with ordinary decoding.

The framework adopted for universality in this paper is based on the notion of compound DMCs, where the encoder and decoder know that the channel law belongs to a certain class $\mathcal{W}$ but do not know which channel law is in effect. This scenario has been widely used in the information theory and communications literature [1], [2], [7] and is particularly useful to model uncertainties about the DMC when a nominal channel model is available. For instance, the nominal model might be the binary-symmetric channel (BSC) with crossover probability $\rho=0.3$, and the compound class might be the set of all BSCs with crossover probability $0.2 \leq \rho \leq 0.4$.

This paper considers decoding with erasures for the compound DMC, with two goals in mind. The first is to construct a broad class of decision rules that can be optimized in an asymptotic Neyman-Pearson sense, analogously to universal hypothesis testing [8]-[10]. The second is to investigate the universality properties of the receiver, in particular conditions under which the exponents coincide with Forney's exponents. We first solve the problem of variable-size list decoders because it is 
simpler, and the solution to the ordinary problem of size-1 lists follows directly. We establish conditions under which our error exponents match Forney's exponents.

Following background material in Section II, the main results are given in Sections III-V. We also observe that in some problems the compound DMC approach is overly rigid and pessimistic. For such problems, we present in Section VI a simple and flexible extension of our method based on the relative minimax principle. In Section VII, we apply our results to a class of BSCs, which yields easily computable and insightful formulas. The paper concludes with a brief discussion in Section VIII. The proofs of the main results are given in the appendices.

\section{A. Notation}

We use uppercase letters for random variables, lowercase letters for individual values, and boldface fonts for sequences. The probability mass function (pmf) of a random variable $X \in \mathcal{X}$ is denoted by $p_{X}=\left\{p_{X}(x), x \in \mathcal{X}\right\}$, the probability of a set $\Omega$ under $p_{X}$ by $P_{X}(\Omega)$, and the expectation operator by $\mathbb{E}$. Entropy of a random variable $X$ is denoted by $H(X)$, and mutual information between two random variables $X$ and $Y$ is denoted by $I(X ; Y)=H(X)-H(X \mid Y)$, or by $I\left(p_{X Y}\right)$ when the dependency on $p_{X Y}$ should be explicit. The Kullback-Leibler divergence between two pmfs $p$ and $q$ is denoted by $D(p \| q)$. All logarithms are in base 2 . We denote by $f^{\prime}$ the derivative of a function $f$.

Denote by $p_{\boldsymbol{x}}$ the type of a sequence $\boldsymbol{x} \in \mathcal{X}^{N}$ ( $p_{\boldsymbol{x}}$ is an empirical pmf over $\mathcal{X}$ ) and by $T_{\boldsymbol{x}}$ the type class associated with $p_{\boldsymbol{x}}$, i.e., the set of all sequences of type $p_{\boldsymbol{x}}$. Likewise, denote by $p_{\boldsymbol{x} y}$ the joint type of a pair of sequences $(\boldsymbol{x}, \boldsymbol{y}) \in \mathcal{X}^{N} \times \mathcal{Y}^{N}$ (an empirical pmf over $\mathcal{X} \times \mathcal{Y}$ ) and by $T_{\boldsymbol{x} y}$ the type class associated with $p_{\boldsymbol{x} y}$, i.e., the set of all sequences of type $p_{\boldsymbol{x} \boldsymbol{y}}$. The conditional type $p_{\boldsymbol{y} \mid \boldsymbol{x}}$ of a pair of sequences $(\boldsymbol{x}, \boldsymbol{y})$ is defined as $p_{\boldsymbol{x} \boldsymbol{y}}(x, y) / p_{\boldsymbol{x}}(x)$ for all $x \in \mathcal{X}$ such that $p_{\boldsymbol{x}}(x)>0$. The conditional type class $T_{\boldsymbol{y} \mid \boldsymbol{x}}$ is the set of all sequences $\tilde{\boldsymbol{y}}$ such that $(\boldsymbol{x}, \tilde{\boldsymbol{y}}) \in T_{\boldsymbol{x} \boldsymbol{y}}$. We denote by $H(\boldsymbol{x})$ the entropy of the pmf $p_{\boldsymbol{x}}$ and by $H(\boldsymbol{y} \mid \boldsymbol{x})$ and $I(\boldsymbol{x} ; \boldsymbol{y})$ the conditional entropy and the mutual information for the joint pmf $p_{\boldsymbol{x} y}$, respectively. Recall that [1]

$$
\begin{aligned}
(N+1)^{-|\mathcal{X}|} 2^{N H(\boldsymbol{x})} & \leq\left|T_{\boldsymbol{x}}\right| \leq 2^{N H(\boldsymbol{x})} \\
(N+1)^{-|\mathcal{X}||\mathcal{Y}|} 2^{N H(\boldsymbol{y} \mid \boldsymbol{x})} & \leq\left|T_{\boldsymbol{y} \mid \boldsymbol{x}}\right| \leq 2^{N H(\boldsymbol{y} \mid \boldsymbol{x})} .
\end{aligned}
$$

We let $\mathcal{P}_{X}$ and $\mathcal{P}_{X}^{[N]}$ represent the set of all pmfs and empirical pmfs, respectively, for a random variable $X$. Likewise, $\mathcal{P}_{Y \mid X}$ and $\mathcal{P}_{Y \mid X}^{[N]}$ denote the set of all conditional pmfs and all empirical conditional pmfs, respectively, for a random variable $Y$ given $X$. The relation $f(N) \sim g(N)$ denotes asymptotic equality: $\lim _{N \rightarrow \infty} \frac{f(N)}{g(N)}=1$. The relations $f(N) \doteq g(N)$ and $f(N) \dot{\leq} g(N)$ denote equality and inequality on the exponential scale: $\lim _{N \rightarrow \infty} \frac{1}{N} \ln \frac{f(N)}{g(N)}=0$ and $\lim _{N \rightarrow \infty} \frac{1}{N} \ln \frac{f(N)}{g(N)} \leq 0$, respectively. We denote by $\mathbb{1}_{\{x \in \Omega\}}$ the indicator function of a set $\Omega$ and define $|t|^{+} \triangleq \max (0, t)$ and $\exp _{2}(t) \triangleq 2^{t}$. We adopt the notational convention that the minimum of a function over an empty set is $+\infty$.

The function-ordering notation $F \preceq G$ indicates that $F(t) \leq$ $G(t)$ for all $t$. Similarly, $F \succeq G$ indicates that $F(t) \geq G(t)$ for all $t$.

\section{DECODING With ERASURE AND List OPTIONS}

\section{A. Maximum-Likelihood Decoding}

In his 1968 paper [3], Forney studied the following erasure/list decoding problem. A length- $N$, rate- $R$ code $\mathcal{C}=$ $\{\boldsymbol{x}(m), m \in \mathcal{M}\}$ is selected, where $\mathcal{M}=\left\{1,2, \ldots, 2^{N R}\right\}$ is the message set and each codeword $\boldsymbol{x}(m) \in \mathcal{X}^{N}$. Upon selection of a message $m$, the corresponding $\boldsymbol{x}(m)$ is transmitted over a DMC $p_{Y \mid X}: \mathcal{X} \rightarrow \mathcal{Y}$. A set of decoding regions $\mathcal{D}_{m} \subseteq \mathcal{Y}^{N}, m \in \mathcal{M}$ is defined, and the decoder returns $\hat{m}=g(\boldsymbol{y})$ if and only if $\boldsymbol{y} \in \mathcal{D}_{m}$. For ordinary decoding, $\left\{\mathcal{D}_{m}, m \in \mathcal{M}\right\}$ form a partition of $\mathcal{Y}^{N}$. When an erasure option is introduced, the decision space is extended to $\mathcal{M} \cup \emptyset$, where $\emptyset$ denotes the erasure symbol. The erasure region $\mathcal{D}_{\emptyset}$ is the complement of $\cup_{m \in \mathcal{M}} \mathcal{D}_{m}$ in $\mathcal{Y}^{N}$. An undetected error arises if $m$ was transmitted but $\boldsymbol{y}$ lies in the decoding region of some other message $i \neq m$. This event is given by

$$
\mathcal{E}_{i}=\left\{(m, \boldsymbol{y}): \boldsymbol{y} \in \underset{i \in \mathcal{M} \backslash\{m\}}{\bigcup} \mathcal{D}_{i}\right\}
$$

where the subscript $i$ stands for "incorrect message." Hence

$$
\begin{aligned}
\operatorname{Pr}\left[\mathcal{E}_{i}\right] & =\frac{1}{|\mathcal{M}|} \sum_{m \in \mathcal{M}} \sum_{\boldsymbol{y} \in \bigcup_{i \in \mathcal{M} \backslash\{m\}} p_{i}} p_{Y \mid X}^{N}(\boldsymbol{y} \mid \boldsymbol{x}(m)) \\
& =\frac{1}{|\mathcal{M}|} \sum_{m \in \mathcal{M}} \sum_{i \in \mathcal{M} \backslash\{m\}} \sum_{\boldsymbol{y} \in \mathcal{D}_{i}} p_{Y \mid X}^{N}(\boldsymbol{y} \mid \boldsymbol{x}(m))
\end{aligned}
$$

where the second equality holds because the decoding regions are disjoint.

The erasure event is given by

$$
\mathcal{E}_{\emptyset}=\left\{(m, \boldsymbol{y}): \boldsymbol{y} \in \mathcal{D}_{\emptyset}\right\}
$$

and has probability

$$
\operatorname{Pr}\left[\mathcal{E}_{\emptyset}\right]=\frac{1}{|\mathcal{M}|} \sum_{m \in \mathcal{M}} \sum_{\boldsymbol{y} \in \mathcal{D}_{\emptyset}} p_{Y \mid X}^{N}(\boldsymbol{y} \mid \boldsymbol{x}(m)) .
$$

The total error event is given by $\mathcal{E}_{\text {err }}=\mathcal{E}_{i} \cup \mathcal{E}_{\emptyset}$. The decoder is generally designed so that $\operatorname{Pr}\left[\mathcal{E}_{i}\right] \ll \operatorname{Pr}\left[\mathcal{E}_{\emptyset}\right]$, so $\operatorname{Pr}\left[\mathcal{E}_{\text {err }}\right] \approx$ $\operatorname{Pr}\left[\mathcal{E}_{\emptyset}\right]$.

Analogously to the Neyman-Pearson problem, one wishes to design the decoding regions to obtain an optimal tradeoff between $\operatorname{Pr}\left[\mathcal{E}_{i}\right]$ and $\operatorname{Pr}\left[\mathcal{E}_{\emptyset}\right]$. Forney proved that the class of decision rules, given in (2.4) at the bottom of the page, is optimal, where $T \geq 0$ is a free parameter trading off $\operatorname{Pr}\left[\mathcal{E}_{i}\right]$ against $\operatorname{Pr}\left[\mathcal{E}_{\emptyset}\right]$. The nonnegativity constraint on $T$ ensures that $\hat{m}$ is uniquely defined for any given $\boldsymbol{y}$. There is no other decision rule that yields simultaneously a lower value for $\operatorname{Pr}\left[\mathcal{E}_{i}\right]$ and for $\operatorname{Pr}\left[\mathcal{E}_{\emptyset}\right]$.

$$
g_{M L}(\boldsymbol{y})=\left\{\begin{array}{lll}
\hat{m} & : & \text { if } p_{Y \mid X}^{N}(\boldsymbol{y} \mid \boldsymbol{x}(\hat{m}))>e^{N T} \sum_{i \neq \hat{m}} p_{Y \mid X}^{N}(\boldsymbol{y} \mid \boldsymbol{x}(i)) \\
\emptyset & : & \text { else }
\end{array}\right.
$$


A conceptually simple (but suboptimal) alternative to (2.4) is (2.5) shown at the bottom of the page, where the decision is made based on the two highest likelihood scores.

If one chooses $T<0$, there is generally more than one value of $\hat{m}$ that satisfies (2.4), and $g_{M L}$ may be viewed as a list decoder that returns the list of all such $\hat{m}$. Denote by $N_{i}$ the number of incorrect messages on the list. Since the decoding regions $\left\{\mathcal{D}_{m}, m \in \mathcal{M}\right\}$ overlap, the average number of incorrect messages in the list

$$
\mathbb{E}\left[N_{i}\right]=\frac{1}{|\mathcal{M}|} \sum_{m \in \mathcal{M}} \sum_{i \in \mathcal{M} \backslash\{m\}} \sum_{\boldsymbol{y} \in \mathcal{D}_{i}} p_{Y \mid X}^{N}(\boldsymbol{y} \mid \boldsymbol{x}(m)),
$$

no longer coincides with $\operatorname{Pr}\left[\mathcal{E}_{i}\right]$ in (2.2).

For the rule (2.4) applied to symmetric channels, Forney showed that the following error exponents are achievable for all $\Delta$ such that $R^{\text {conj }} \leq R+\Delta \leq C$ :

$$
\begin{aligned}
& E_{i}(R, \Delta)=E_{\mathrm{sp}}(R)+\Delta \\
& E_{\emptyset}(R, \Delta)=E_{\mathrm{sp}}(R+\Delta)
\end{aligned}
$$

where $E_{\mathrm{sp}}(R)$ is the sphere-packing exponent, and $R^{\text {conj }}$ is the conjugate rate, defined as the rate for which the slope of $E_{\mathrm{sp}}(\cdot)$ is the reciprocal of the slope at rate $R$

$$
E_{\mathrm{sp}}^{\prime}\left(R^{\mathrm{conj}}\right)=\frac{1}{E_{\mathrm{sp}}^{\prime}(R)} .
$$

The exponents of (2.7) are achieved using independent and identically distributed (i.i.d.) codes.

\section{B. Universal Decoding}

When the channel law $p_{Y \mid X}$ is unknown, maximum-likelihood decoding cannot be used. For constant-composition codes with type $p_{X}$, the MMI decoder takes the form

$$
g_{\mathrm{MMI}}(\boldsymbol{y})=\arg \max _{i \in \mathcal{M}} I(\boldsymbol{x}(i) ; \boldsymbol{y}) .
$$

Csiszár and Körner [1, pp. 174-178] extended the MMI decoder to include an erasure option, using the decision rule (2.10) also shown at the bottom of the page, where $\Delta \geq 0$ and $\lambda>1$.
They derived the following error exponents for the resulting undetected-error and erasure events:

$$
\left\{E_{r, \lambda}\left(R, p_{X}, p_{Y \mid X}\right)+\Delta, E_{r, 1 / \lambda}\left(R+\Delta, p_{X}, p_{Y \mid X}\right)\right\},
$$

$\forall p_{Y \mid X}$

where

$$
\begin{aligned}
& E_{r, \lambda}\left(R, p_{X}, p_{Y \mid X}\right) \\
& \quad=\min _{\tilde{p}_{Y \mid X}}\left\{D\left(\tilde{p}_{Y \mid X} \| p_{Y \mid X} \mid p_{X}\right)+\lambda\left|I\left(p_{X}, \tilde{p}_{Y \mid X}\right)-R\right|^{+}\right\} .
\end{aligned}
$$

While $\Delta$ and $\lambda$ are tradeoff parameters, they did not mention whether the decision rule (2.10) satisfies any Neyman-Pearson type optimality criterion.

A different approach was recently proposed by Merhav and Feder [6]. They raised the possibility that the achievable pairs of undetected-error and erasure exponents might be smaller than in the known-channel case and proposed a decision rule based on the competitive minimax principle. This rule is parameterized by a scalar parameter $0 \leq \xi \leq 1$ which represents a fraction of the optimal exponents (for the known-channel case) that their decoding procedure is guaranteed to achieve. Decoding involves explicit maximization of a cost function over the compound DMC family, analogously to a generalized likelihood ratio test (GLRT). The rule coincides with the GLRT when $\xi=0$, but the choice of $\xi$ can be optimized. They conjectured that the highest achievable $\xi$ is lower than 1 in general, and derived a computable lower bound on that value.

\section{III. $\mathcal{F}$-MMI CLASS OF DECODERS}

\section{A. Decoding Rule}

Assume that random constant-composition codes with type $p_{X}$ are used, and that the DMC $p_{Y \mid X}$ belongs to a compact subset $\mathcal{W}$ of $\mathcal{P}_{Y \mid X}$. The decoder knows $\mathcal{W}$ but not which $p_{Y \mid X}$ is in effect.

Analogously to (2.10), our proposed decoding rule is a test based on the empirical mutual informations for each candidate message and its highest scoring competitor. Let $\mathcal{F}$ be the class of continuous, nondecreasing functions $F:\left[-R, H\left(p_{X}\right)-R\right] \rightarrow$ $\mathbb{R}$. The decision rule indexed by $F \in \mathcal{F}$ takes the form of (3.1) shown at the bottom of the page. Given a candidate message $\hat{m}$,

$$
g_{M L, 2}(\boldsymbol{y})=\left\{\begin{array}{lll}
\hat{m} & : & \text { if } p_{Y \mid X}^{N}(\boldsymbol{y} \mid \boldsymbol{x}(\hat{m}))>e^{N T} \max _{i \neq \hat{m}} p_{Y \mid X}^{N}(\boldsymbol{y} \mid \boldsymbol{x}(i)) \\
\emptyset: & \text { else }
\end{array}\right.
$$

$$
g_{\lambda, \Delta}(\boldsymbol{y})=\left\{\begin{array}{lll}
\hat{m} & : & \text { if } I(\boldsymbol{x}(\hat{m}) ; \boldsymbol{y})>R+\Delta+\lambda \max _{i \neq \hat{m}}|I(\boldsymbol{x}(i) ; \boldsymbol{y})-R|^{+} \\
\emptyset \quad: & \text { else }
\end{array}\right.
$$

$$
g_{F}(\boldsymbol{y})=\left\{\begin{array}{lll}
\hat{m} & : & \text { if } I(\boldsymbol{x}(\hat{m}) ; \boldsymbol{y})>R+\max _{i \neq \hat{m}} F(I(\boldsymbol{x}(i) ; \boldsymbol{y})-R) \\
\emptyset & : & \text { else. }
\end{array}\right.
$$


the function $F$ weighs the score of the best competing codeword. The decoder outputs $\hat{m}$ if its empirical mutual information score is large enough relative to its strongest competitor, as weighted by $F .{ }^{1}$ Since $0 \leq I(\boldsymbol{x}(i) ; \boldsymbol{y}) \leq H\left(p_{X}\right)$, all values of $F(t)$ for $t \notin\left[-R, H\left(p_{X}\right)-R\right]$ are equivalent in terms of the decision rule (3.1).

The choice $F(t)=t$ results in the MMI decoding rule (2.9), and

$$
F(t)=\Delta+\lambda|t|^{+}
$$

(two-parameter family of functions) results in the Csiszár-Körner rule (2.10).

One may further require that $F(t) \geq t$ to guarantee that $\hat{m}=\arg \max _{i} I(\boldsymbol{x}(i) ; \boldsymbol{y})$, as can be verified by direct substitution into (3.1). In this case, the decision is whether the decoder should output the highest scoring message or output an erasure decision.

When the restriction $F(t) \geq t$ is not imposed, the decision rule (3.1) is ambiguous because more than one $\hat{m}$ could satisfy the inequality in (3.1). Then (3.1) may be viewed as a list decoder that returns the list of all such $\hat{m}$, similarly to (2.4).

The Csiszár-Körner decision rule parameterized by $F$ in (3.2) is nonambiguous for $\lambda \geq 1$. Note that there is an error in Theorem 5.11 and Corollary 5.11A of [1, p. 175], where the condition $\lambda>0$ should be replaced with $\lambda \geq 1[11]$.

In the limit as $\lambda \downarrow 0,(3.2)$ leads to the simple decoder that lists all messages whose empirical mutual information score exceeds $R+\Delta$. If a list decoder is not desired, a simple variation on (3.2) when $0<\lambda<1$ is

$$
F(t)=\left\{\begin{array}{lll}
\Delta+\lambda|t|^{+} & : & t \leq \frac{\Delta}{1-\lambda} \\
t & : & \text { else. }
\end{array}\right.
$$

It is also worth noting that the function $F(t)=\Delta+t$ may be thought of as an empirical version of Forney's suboptimal decoding rule $(2.5)$, with $T=\Delta$. Indeed, using the identity $I(\boldsymbol{x} ; \boldsymbol{y})=H(\boldsymbol{y})-H(\boldsymbol{y} \mid \boldsymbol{x})$ and viewing the negative empirical equivocation

$$
-H(\boldsymbol{y} \mid \boldsymbol{x})=\sum_{x, y} p_{\boldsymbol{x} \boldsymbol{y}}(x, y) \ln p_{\boldsymbol{y} \mid \boldsymbol{x}}(y \mid x)
$$

as an empirical version of the normalized loglikelihood

$$
\frac{1}{N} \ln p_{Y \mid X}^{N}(\boldsymbol{y} \mid \boldsymbol{x})=\sum_{x, y} p_{\boldsymbol{x} \boldsymbol{y}}(x, y) \ln p_{Y \mid X}(y \mid x),
$$

${ }^{1}$ Note that the function $F$ could have been defined slightly differently as $G(t)=R+F(t-R)$. The right side of (3.1) would be written more compactly as $\max _{i \neq \hat{m}} G(I(\boldsymbol{x}(i) ; \boldsymbol{y}))$ but the resulting expressions (4.3) and (4.7) for random-coding exponents would be somewhat less elegant. we may rewrite (2.5) and (3.1) respectively as(3.3) at the bottom of the page, and

$$
g_{F}(\boldsymbol{y})= \begin{cases}\hat{m} & : \text { if }-H(\boldsymbol{y} \mid \boldsymbol{x}(\hat{m}))>\Delta+\max _{i \neq \hat{m}}[-H(\boldsymbol{y} \mid \boldsymbol{x}(i))] \\ \emptyset \quad: \text { else. }\end{cases}
$$

While this observation does not imply $F(t)=\Delta+t$ is an optimal choice for $F$, one might intuitively expect optimality in some regime.

\section{B. Error Exponents}

For a random-coding strategy using constant-composition codes with type $p_{X}$, the expected number of incorrect messages on the list $\mathbb{E}\left[N_{i}\right]$ and the erasure probability, $\operatorname{Pr}\left[\mathcal{E}_{\emptyset}\right]$ may be viewed as functions of $R, p_{X}, p_{Y \mid X}$, and $F$.

Definition 3.1: A pair

$$
\left\{E_{i}\left(R, p_{X}, p_{Y \mid X}, F\right), E_{\emptyset}\left(R, p_{X}, p_{Y \mid X}, F\right)\right\}
$$

of incorrect-message and erasure exponents is said to be universally attainable over $\mathcal{W}$ for random codes of rate $R$ and type $p_{X}$ if the expected number of incorrect messages on the list and the erasure probability satisfy

$$
\begin{array}{r}
\mathbb{E}\left[N_{i}\right] \leq \exp _{2}\left\{-N\left[E_{i}\left(R, p_{X}, p_{Y \mid X}, F\right)-\epsilon\right]\right\} \\
\operatorname{Pr}\left[\mathcal{E}_{\emptyset}\right] \leq \exp _{2}\left\{-N\left[E_{\emptyset}\left(R, p_{X}, p_{Y \mid X}, F\right)-\epsilon\right]\right\}, \\
\forall p_{Y \mid X} \in \mathcal{W}
\end{array}
$$

for any $\epsilon>0$ and $N$ greater than some $N_{0}(\epsilon)$.

The worst case exponents (over all $p_{Y \mid X} \in \mathcal{W}$ ) are denoted by

$$
\begin{aligned}
& E_{i}\left(R, p_{X}, \mathcal{W}, F\right) \triangleq \min _{p_{Y \mid X} \in \mathcal{W}} E_{i}\left(R, p_{X}, p_{Y \mid X}, F\right) \\
& E_{\emptyset}\left(R, p_{X}, \mathcal{W}, F\right) \triangleq \min _{p_{Y \mid X} \in \mathcal{W}} E_{\emptyset}\left(R, p_{X}, p_{Y \mid X}, F\right) .
\end{aligned}
$$

Our problem is to maximize the erasure exponent $E_{\emptyset}\left(R, p_{X}, \mathcal{W}, F\right)$ subject to the constraint that the incorrect-message exponent $E_{i}\left(R, p_{X}, \mathcal{W}, F\right)$ is at least equal to some prescribed value $\alpha$. This is an asymptotic Neyman-Pearson problem. We shall focus on the regime of practical interest where erasures are more acceptable than undetected errors

$$
E_{\emptyset}\left(R, p_{X}, \mathcal{W}, F\right) \leq E_{i}\left(R, p_{X}, \mathcal{W}, F\right) .
$$

We emphasize that asymptotic Neyman-Pearson optimality of the decision rule holds only in a restricted sense, namely, with respect to the $\mathcal{F}$-MMI class (3.1).

$$
g_{M L, 2}(\boldsymbol{y})=\left\{\begin{array}{lll}
\hat{m} & : & \text { if } \frac{1}{N} \ln p_{Y \mid X}^{N}(\boldsymbol{y} \mid \boldsymbol{x}(\hat{m}))>T+\max _{i \neq \hat{m}} \frac{1}{N} \ln p_{Y \mid X}^{N}(\boldsymbol{y} \mid \boldsymbol{x}(i)) \\
\emptyset & : \quad \text { else }
\end{array}\right.
$$


Specifically, given $R$ and $\mathcal{W}$, we seek the solution to the constrained optimization problem

$$
\begin{aligned}
& E_{\emptyset}^{*}(R, \mathcal{W}, \alpha) \\
& \triangleq \max _{p_{X}} \max _{F \in \mathcal{F}\left(R, p_{X}, \mathcal{W}, \alpha\right)} \min _{p_{Y \mid X} \in \mathcal{W}} E_{\emptyset}\left(R, p_{X}, p_{Y \mid X}, F\right)
\end{aligned}
$$

where $\mathcal{F}\left(R, p_{X}, \mathcal{W}, \alpha\right)$ is the set of functions $F$ that satisfy

$$
\min _{p_{Y \mid X} \in \mathcal{W}} E_{i}\left(R, p_{X}, p_{Y \mid X}, F\right) \geq \alpha
$$

as well as the continuity and monotonicity conditions mentioned above (3.1).

If we were able to choose $F$ as a function of $p_{Y \mid X}$, we would do at least as well as in (3.9) and achieve the erasure exponent

$$
\begin{aligned}
E_{\emptyset}^{* *}(R, \mathcal{W}, \alpha) \triangleq & \max _{p_{X}} \min _{p_{Y \mid X} \in \mathcal{W}} \max _{F \in \mathcal{F}\left(R, p_{X}, p_{Y \mid X}, \alpha\right)} \\
& E_{\emptyset}\left(R, p_{X}, p_{Y \mid X}, F\right) \\
\geq & E_{\emptyset}^{*}(R, \mathcal{W}, \alpha) .
\end{aligned}
$$

The max min max order for this optimization corresponds to a widely used communications scenario without feedback where each player only knows the strategy of the previous player(s) and so the encoder does not know the channel law, but the decoder does. We shall be particularly interested in characterizing $(R, \mathcal{W}, \alpha)$ for which the decoder incurs no penalty for not knowing $p_{Y \mid X}$, i.e.,

- equality holds in (3.11), and

- the optimal exponents $E_{i}\left(R, p_{X}, p_{Y \mid X}, F\right)$ and $E_{\emptyset}\left(R, p_{X}, p_{Y \mid X}, F\right)$ in (3.10) and (3.9) coincide with Forney's exponents in (2.7) for all $p_{Y \mid X} \in \mathcal{W}$, the second property being stronger than the first.

\section{Basic Properties of $F$}

To simplify the derivations, it is convenient to slightly strengthen the requirement that $F$ be nondecreasing, and work with (strictly) increasing functions $F$ instead. Then the maxima over $F$ in (3.9) and (3.11) are replaced with suprema, but of course their value remains the same.

To each monotonically increasing function $F \in \mathcal{F}$ corresponds an inverse $F^{-1}$, such that

$$
F(t)=u \Leftrightarrow F^{-1}(u)=t .
$$

Since $F$ is continuous and monotonically increasing, elementary properties satisfied by $F^{-1}$ include:

(P1) $F^{-1}$ is continuous and increasing over its range.

(P2) If $F \preceq G$, then $F^{-1} \succeq G^{-1}$.

(P3) $G(t)=F(t)+\Delta \Leftrightarrow \bar{G}^{-1}(t)=F^{-1}(t-\Delta)$.

(P4) If $F$ is differentiable, then $\frac{d F(t)}{d t}=1 / \frac{d F^{-1}(t)}{d t}$.

(P5) If $F$ is convex, then $F^{-1}$ is concave.

(P6) The domain of $F^{-1}$ is the range of $F$, and vice versa.

The function $F^{-1}$ that appears in the statement of our main results can still be defined if $F$ is not strictly increasing, but some care must be taken, in particular in the definition of the domain of $F^{-1}$. For instance, given parameters $\lambda, \epsilon>0$, the function

$$
F(t)=\left\{\begin{array}{lll}
\Delta+\lambda t & : & 0 \leq t \leq H\left(p_{X}\right)-R \\
\Delta+\epsilon t & : & -R \leq t \leq 0
\end{array}\right.
$$

is increasing and converges in the sup norm to (3.2) as $\epsilon \downarrow 0$. The inverse of (3.12) is given by

$$
F^{-1}(t)=\left\{\begin{array}{lll}
\lambda^{-1}(t-\Delta) & : & 0 \leq t-\Delta \leq \lambda\left[H\left(p_{X}\right)-R\right] \\
\epsilon^{-1}(t-\Delta) & : & -\epsilon R \leq t-\Delta \leq 0 .
\end{array}\right.
$$

In the limit case $\epsilon=0$, the domain of $F^{-1}$ reduces to $[\Delta, \Delta+$ $\left.\lambda\left(H\left(p_{X}\right)-R\right)\right]$, and ${ }^{2}$

$$
F^{-1}(t)=\frac{t-\Delta}{\lambda}, \text { for } t \geq \Delta .
$$

In the limit case $\lambda=\epsilon=0$, corresponding to $F(t) \equiv \Delta$, the domain of $F^{-1}$ reduces to the singleton $\{\Delta\}$.

\section{RANDOM-CODING AND SPHERE-PACKING EXPONENTS}

The sphere-packing exponent for channel $p_{Y \mid X}$ is defined as $E_{\mathrm{sp}}\left(R, p_{X}, p_{Y \mid X}\right)$

$$
\triangleq \min _{\tilde{p}_{Y \mid X}: I\left(p_{X}, \tilde{p}_{Y \mid X}\right) \leq R} D\left(\tilde{p}_{Y \mid X} \| p_{Y \mid X} \mid p_{X}\right)
$$

and as $\infty$ if the minimization above is over an empty set. The function $E_{\mathrm{sp}}\left(R, p_{X}, p_{Y \mid X}\right)$ is convex, nonincreasing, and continuous in $p_{Y \mid X}$. The first two properties are given in [1, Lemma 5.4]. The third property holds because the minimand of (4.1) may be viewed as a functional $\Phi\left(\tilde{p}_{Y \mid X}, p_{Y \mid X}\right)$, and the left side of (4.1) as a functional $E\left(p_{Y \mid X}\right)=\min _{\tilde{p}_{Y \mid X}} \Phi\left(\tilde{p}_{Y \mid X}, p_{Y \mid X}\right)$. Since $\Phi$ is continuous in $\left(\tilde{p}_{Y \mid X}, p_{Y \mid X}\right)$ with respect to $L^{1}$ norm, (4.1) is also a continuous functional of $p_{Y \mid X}$.

The sphere-packing exponent for class $\mathcal{W}$ is defined as

$$
E_{\mathrm{sp}}\left(R, p_{X}, \mathcal{W}\right) \triangleq \min _{p_{Y \mid X} \in \mathcal{W}} E_{\mathrm{sp}}\left(R, p_{X}, p_{Y \mid X}\right) .
$$

The function $E_{\mathrm{sp}}\left(R, p_{X}, \mathcal{W}\right)$ is continuous and nonincreasing in $R$. In some cases, $E_{\mathrm{sp}}\left(R, p_{X}, \mathcal{W}\right)$ is also convex and continuously differentiable in $R$, e.g., when the same $p_{Y \mid X}$ achieves the minimum in (4.2) at all rates. Denote by $R_{\infty}\left(p_{X}, \mathcal{W}\right)$ the infimum of the rates $R$ such that $E_{\mathrm{sp}}\left(R, p_{X}, \mathcal{W}\right)<\infty$, and by

$$
I\left(p_{X}, \mathcal{W}\right) \triangleq \min _{p_{Y \mid X} \in \mathcal{W}} I\left(p_{X}, p_{Y \mid X}\right)
$$

the supremum of $R$ such that $E_{\mathrm{sp}}\left(R, p_{X}, \mathcal{W}\right)>0$.

The modified random coding exponent for channel $p_{Y \mid X}$ and for class $\mathcal{W}$ are, respectively, defined as

$$
\begin{aligned}
E_{r, F}\left(R, p_{X}, p_{Y \mid X}\right) \triangleq \min _{\tilde{p}_{Y \mid X}}[ & D\left(\tilde{p}_{Y \mid X} \| p_{Y \mid X} \mid p_{X}\right) \\
& \left.+F\left(I\left(p_{X}, \tilde{p}_{Y \mid X}\right)-R\right)\right]
\end{aligned}
$$

\footnotetext{
${ }^{2}$ Any nondecreasing $F$ can be written as the limit of a sequence of increasing functions converging in the sup norm. Since $F$ is defined over a finite interval, this implies convergence in the $L^{1}$ norm. The sequence of inverse functions converges in the $L^{1}$ norm to a right-continuous function which may be defined as $F^{-1}$. Since $E_{r, F}$ defined in (4.3) is continuous with respect to $F$ in the $L^{1}$ norm, it follows that Propositions 4.1 and 4.2 hold for nondecreasing $F$ and not just for increasing $F$.
} 


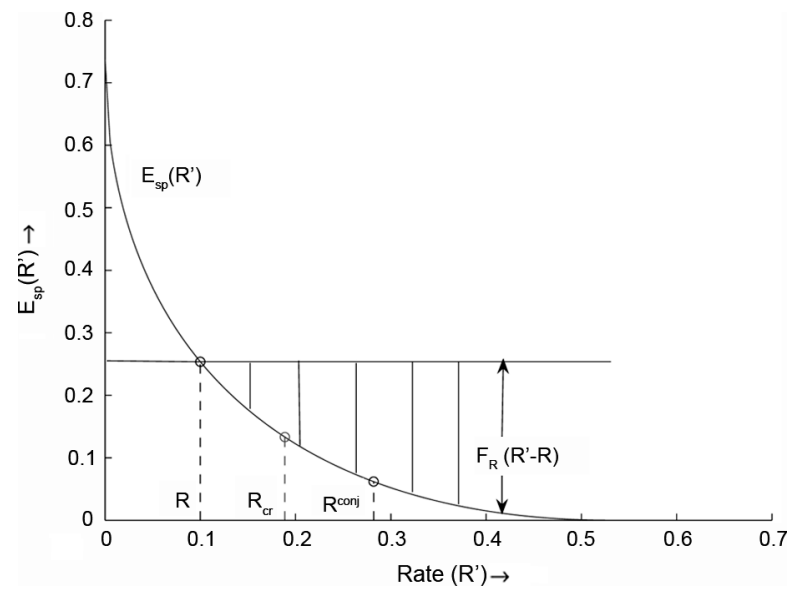

Fig. 1. A graphical depiction of the function $F_{R, p_{X}, \mathcal{W}}\left(R^{\prime}-R\right)$ versus $R^{\prime}$ when $R=0.1, \mathcal{W}$ is the family of BSCs with crossover probability $\rho \leq 0.1$ (capacity $C(\rho) \geq 0.53$ ), and $p_{X}$ is the uniform pmf over $\{0,1\}$. The function is evaluated from the sphere-packing exponent $E_{\mathrm{sp}}\left(R^{\prime}\right)$ for class $\mathcal{W}$.

and

$$
E_{r, F}\left(R, p_{X}, \mathcal{W}\right) \triangleq \min _{p_{Y \mid X} \in \mathcal{W}} E_{r, F}\left(R, p_{X}, p_{Y \mid X}\right)
$$

We may view the minimand of (4.3) as a functional $\Phi\left(F, \tilde{p}_{Y \mid X}\right)$ and the left side of (4.3) as a functional

$$
E(F)=\min _{p_{Y \mid X}} \Phi\left(F, \tilde{p}_{Y \mid X}\right)
$$

Since $\Phi$ is continuous in $\left(F, \tilde{p}_{Y \mid X}\right),(4.3)$ is a continuous functional of $F$. When $F(t)=|t|^{+},(4.3)$ is just the usual random coding exponent.

Define the function

$$
F_{R, p_{X}, \mathcal{W}}(t) \triangleq E_{\mathrm{sp}}\left(R, p_{X}, \mathcal{W}\right)-E_{\mathrm{sp}}\left(R+t, p_{X}, \mathcal{W}\right)
$$

which is depicted in Fig. 1 for a BSC example to be analyzed in Section VII. This function is increasing for $R_{\infty}\left(p_{X}, \mathcal{W}\right)-$ $R \leq t \leq I\left(p_{X}, \mathcal{W}\right)-R$ and satisfies the following elementary properties:

$$
\begin{aligned}
F_{R, p_{X}, \mathcal{W}}(0) & =0 \\
F_{R, p_{X}, \mathcal{W}}^{\prime}(t) & =-E_{\mathrm{sp}}^{\prime}\left(R+t, p_{X}, \mathcal{W}\right)
\end{aligned}
$$

$E_{\mathrm{sp}}\left(R^{\prime}, p_{X}, \mathcal{W}\right)+F_{R, p_{X}, \mathcal{W}}\left(R^{\prime}-R\right) \equiv E_{\mathrm{sp}}\left(R, p_{X}, \mathcal{W}\right)$

If $E_{\mathrm{sp}}\left(R, p_{X}, \mathcal{W}\right)$ is convex in $R$, then $F_{R, p_{X}, \mathcal{W}}(t)$ is concave in $t$.

Proposition 4.1: The modified random coding exponent $E_{r, F}\left(R, p_{X}, p_{Y \mid X}\right)$ satisfies the following properties.

(i) $E_{r, F}\left(R, p_{X}, p_{Y \mid X}\right)$ is decreasing in $R$ for all $R \leq$ $I\left(p_{X}, p_{Y \mid X}\right)$.

(ii) If $F \preceq G$, then

$$
E_{r, F}\left(R, p_{X}, p_{Y \mid X}\right) \leq E_{r, G}\left(R, p_{X}, p_{Y \mid X}\right) .
$$

(iii) $E_{r, F}\left(R, p_{X}, p_{Y \mid X}\right)$ is related to the sphere packing exponent as follows:

$$
\begin{aligned}
& E_{r, F}\left(R, p_{X}, p_{Y \mid X}\right) \\
& \quad=\min _{R^{\prime}}\left[E_{\mathrm{sp}}\left(R^{\prime}, p_{X}, p_{Y \mid X}\right)+F\left(R^{\prime}-R\right)\right] .
\end{aligned}
$$

(iv) The preceding properties hold with $\mathcal{W}$ in place of $p_{Y \mid X}$ in the arguments of the functions $E_{r, F}, E_{\mathrm{sp}}$, and $I$.

The proof of these properties is given in Appendix A. Part (iii) is a variation on Lemma 5.4 and its corollary in [1, p. 168]. Also note that while $E_{r, F}\left(R, p_{X}, p_{Y \mid X}\right)$ is convex in $R$ for some choices of $F$, including (3.2), that property does not extend to arbitrary $F$.

Proposition 4.2: The following pair of incorrect-message and erasure exponents are universally attainable over $\mathcal{W}$ (recall Definition 3.1) under the decision rule (3.1):

$$
\begin{aligned}
& E_{i}\left(R, p_{X}, p_{Y \mid X}, F\right)=E_{r, F}\left(R, p_{X}, p_{Y \mid X}\right) \\
& E_{\emptyset}\left(R, p_{X}, p_{Y \mid X}, F\right)=E_{r,\left|F^{-1}\right|^{+}}\left(R, p_{X}, p_{Y \mid X}\right), \quad \forall F \in \mathcal{F} .
\end{aligned}
$$

Proof: See Appendix B.

If $F(t)=|t|^{+}$, then $F^{-1}(t)=t$ for $t \geq 0$, and both (4.8) and (4.9) reduce to the ordinary random-coding exponent.

If the channel is not reliable enough in the sense that $I\left(p_{X}, p_{Y \mid X}\right)<R+F(0)$, then

$$
F^{-1}\left(I\left(p_{X}, p_{Y \mid X}\right)-R\right) \leq F^{-1}(F(0))=0
$$

and from (4.9) we obtain $E_{\emptyset}\left(R, p_{X}, p_{Y \mid X}, F\right)=0$ because the minimizing $\tilde{p}_{Y \mid X}$ in the expression for $E_{r,\left|F^{-1}\right|^{+}}$is equal to $p_{Y \mid X}$. Checking (3.1), a heuristic interpretation for the zero erasure exponent is that $I(\boldsymbol{x}(m) ; \boldsymbol{y}) \approx I\left(p_{X}, p_{Y \mid X}\right)$ with high probability when $m$ is the transmitted message, and $\max _{i \neq m} I(\boldsymbol{x}(m) ; \boldsymbol{y}) \approx R$ (obtained using (B.4) with $\nu=R$ and the union bound). Hence $m$ fails the test (3.1) with high probability.

\section{W-Optimal ChOice of $F$}

In this section, we view the incorrect-message and erasure exponents as functionals of $|F|^{+}$and examine optimal tradeoffs between them. It is instructive to first consider the one-parameter family

$$
F(t) \equiv \Delta \geq 0
$$

which corresponds to a thresholding rule in (3.1). Using (4.7)-(4.9), we obtain

$$
\begin{aligned}
& E_{i}\left(R, p_{X}, p_{Y \mid X}, F\right)=E_{r, F}\left(R, p_{X}, p_{Y \mid X}\right) \equiv \Delta \\
& E_{\emptyset}\left(R, p_{X}, p_{Y \mid X}, F\right) \equiv E_{\mathrm{sp}}\left(R+\Delta, p_{Y \mid X}\right)
\end{aligned}
$$

where (5.2) holds because the minimum in (4.7) is achieved at $R^{\prime}=I\left(p_{X}, p_{Y \mid X}\right)$, in which case the sphere-packing exponent is 0 ; and (5.3) is obtained by viewing $F(t) \equiv \Delta$ as the limit of a sequence of increasing functions, as described at the end of Section III-C. Two extreme choices for $\Delta$ are 0 and $I\left(p_{X}, \mathcal{W}\right)-$ $R$ because in each case one error exponent is zero and the other one is positive. One would expect that better tradeoffs can be achieved using a broader class of functions $F$, though.

Recalling (3.9), (3.10), and using (4.8) and (4.9), we seek the solution to the following two asymptotic Neyman-Pearson optimization problems. For list decoding, find

$$
E_{\emptyset}^{L}(R, \mathcal{W}, \alpha)=\max _{p_{X}} E_{\emptyset}^{L}\left(R, p_{X}, \mathcal{W}, \alpha\right)
$$


where the cost function

$$
\begin{aligned}
E_{\emptyset}^{L}\left(R, p_{X}, \mathcal{W}, \alpha\right) & \sup _{F \in \mathcal{F}^{L}\left(R, p_{X}, \mathcal{W}, \alpha\right)} E_{r,\left|F^{-1}\right|^{+}}\left(R, p_{X}, \mathcal{W}\right)
\end{aligned}
$$

and the feasible set $\mathcal{F}^{L}\left(R, p_{X}, \mathcal{W}, \alpha\right)$ is the set of continuous, increasing functions $F$ that yield an incorrect-message exponent at least equal to $\alpha$

$$
E_{i}\left(R, p_{X}, \mathcal{W}, F\right)=E_{r, F}\left(R, p_{X}, \mathcal{W}\right) \geq \alpha .
$$

For classical decoding (list size $\leq 1$ ), find

$$
E_{\emptyset}(R, \mathcal{W}, \alpha)=\max _{p_{X}} E_{\emptyset}\left(R, p_{X}, \mathcal{W}, \alpha\right)
$$

where

$$
\begin{aligned}
E_{\emptyset}\left(R, p_{X}, \mathcal{W}, \alpha\right) & \sup _{F \in \mathcal{F}\left(R, p_{X}, \mathcal{W}, \alpha\right)} E_{r,\left|F^{-1}\right|^{+}}\left(R, p_{X}, \mathcal{W}\right)
\end{aligned}
$$

and $\mathcal{F}\left(R, p_{X}, \mathcal{W}, \alpha\right)$ is the subset of functions in $\mathcal{F}^{L}\left(R, p_{X}, \mathcal{W}, \alpha\right)$ that satisfy $F(t) \geq t$, i.e., the decoder outputs at most one message.

Since $\mathcal{F}\left(R, p_{X}, \mathcal{W}, \alpha\right) \subset \mathcal{F}^{L}\left(R, p_{X}, \mathcal{W}, \alpha\right)$, we have

$$
E_{\emptyset}\left(R, p_{X}, \mathcal{W}, \alpha\right) \leq E_{\emptyset}^{L}\left(R, p_{X}, \mathcal{W}, \alpha\right) .
$$

The remainder of this paper focuses on the class of variable-size list decoders associated with $\mathcal{F}^{L}$ because the error exponent tradeoffs are at least as good as those associated with $\mathcal{F}$, and the corresponding error exponents take a more concise form.

Define the critical rate $R_{c r}\left(p_{X}, \mathcal{W}\right)$ as the rate at which the derivative $E_{\mathrm{sp}}^{\prime}\left(\cdot, p_{X}, \mathcal{W}\right)=-1$ (assuming this derivative exists), and

$$
\Delta \triangleq \alpha-E_{\mathrm{sp}}\left(R, p_{X}, \mathcal{W}\right) .
$$

Analogously to (2.8), two rates $R_{1}$ and $R_{2}$ are said to be conjugate given $p_{X}$ and $\mathcal{W}$ if the corresponding slopes of $E_{\mathrm{sp}}\left(\cdot, p_{X}, \mathcal{W}\right)$ are reciprocal of each other

$$
E_{\mathrm{sp}}^{\prime}\left(R_{1}, p_{X}, \mathcal{W}\right)=\frac{1}{E_{\mathrm{sp}}^{\prime}\left(R_{2}, p_{X}, \mathcal{W}\right)} .
$$

The difference $d$ between two conjugate rates uniquely specifies them. We denote the smaller one by $R_{1}(d)$ and the larger one by $R_{2}(d)$, irrespective of the sign of $d$. Hence, $R_{1}(d) \leq R_{c r}\left(p_{X}, \mathcal{W}\right) \leq R_{2}(d)$, with equality when $d=0$. We also denote by $R^{\operatorname{conj}}\left(p_{X}, \mathcal{W}\right)$ the conjugate rate of $R$, as defined by (5.10). The conjugate rate always exists when $R$ is below the critical rate $R_{c r}\left(p_{X}, \mathcal{W}\right)$. If $R$ is above the critical rate and sufficiently large, $R^{\operatorname{conj}}\left(p_{X}, \mathcal{W}\right)$ may not exist. Instead of treating this case separately, we note that this case will be irrelevant because the conjugate rate always appears via the expression $\max \left\{R, R^{\operatorname{conj}}\left(p_{X}, \mathcal{W}\right)\right\}$ in our analysis. This maximum is equal to $R$ if $R>R_{c r}\left(p_{X}, \mathcal{W}\right)$ and is therefore always well defined.

The proofs of Propositions 5.1 and 5.3 and Lemma 5.4 below may be found in Appendices C-E; recall $F_{R, p_{X}, \mathcal{W}}(t)$ was defined in (4.5). The proof of Proposition 5.5 parallels that of Proposition 5.1(ii) and is therefore omitted.
Proposition 5.1: The suprema in (5.5) and (5.8) are respectively achieved by

$$
\begin{aligned}
F^{L *}(t) & =F_{R, p_{X}, \mathcal{W}}(t)+\Delta \\
& =\alpha-E_{\mathrm{sp}}\left(R+t, p_{X}, \mathcal{W}\right)
\end{aligned}
$$

and

$$
F^{*}(t)=\max \left(t, F^{L *}(t)\right) .
$$

The resulting incorrect-message exponent is given by $E_{i}\left(R, p_{X}, \mathcal{W}\right)=\alpha$. The optimal solution is nonunique. In particular, for $t \leq 0$, one can replace $F^{L^{*}}(t)$ by the constant $F^{L *}(0)$ without effect on the error exponents.

The proof of Proposition 5.1 is quite simple and can be separated from the calculation of the error exponents. The main idea is this: If $G \preceq F$, we have

$$
E_{r, G}\left(R, p_{X}, \mathcal{W}\right) \leq E_{r, F}\left(R, p_{X}, \mathcal{W}\right) .
$$

Since $G^{-1} \succeq F^{-1}$, we also have

$$
E_{r, G^{-1}}\left(R, p_{X}, \mathcal{W}\right) \geq E_{r, F^{-1}}\left(R, p_{X}, \mathcal{W}\right) .
$$

Therefore, we seek $F^{*} \in \mathcal{F}^{L}\left(R, p_{X}, \mathcal{W}, \alpha\right)$ such that $F^{*} \preceq F$ for all $F \in \mathcal{F}^{L}\left(R, p_{X}, \mathcal{W}, \alpha\right)$. Such $F^{*}$, assuming it exists, necessarily achieves $E_{\emptyset}^{L}\left(R, p_{X}, \mathcal{W}, \alpha\right)$. The same procedure applies to $\mathcal{F}\left(R, p_{X}, \mathcal{W}, \alpha\right)$.

Corollary 5.2: If $R \geq I\left(p_{X}, \mathcal{W}\right)$, the thresholding rule $F^{L *}(t) \equiv \Delta$ of (5.1) is optimal, and the optimal error exponents are $E_{i}\left(R, p_{X}, \mathcal{W}, \alpha\right)=\Delta$ and $E_{\emptyset}^{L}\left(R, p_{X}, \mathcal{W}, \alpha\right)=0$.

Proof: Since $R \geq I\left(p_{X}, \mathcal{W}\right)$, we have $E_{\mathrm{sp}}\left(R, p_{X}, \mathcal{W}\right)=$ 0 . Hence, from (4.5), $F_{R, p_{X}, \mathcal{W}}(t) \equiv 0$ for all $t \geq 0$. Substituting into (5.11) establishes the optimality of the thresholding rule (5.1). The corresponding error exponents are obtained by minimizing (5.2) and (5.3) over $p_{Y \mid X} \in \mathcal{W}$.

The more elaborate case $R<I\left(p_{X}, \mathcal{W}\right)$ is addressed next.

Proposition 5.3: If $E_{\mathrm{sp}}\left(R, p_{X}, \mathcal{W}\right)$ is convex and differentiable in $R$, the optimal incorrect-message and erasure exponents are related as follows.

(i) For $\left|R^{\operatorname{conj}}\left(p_{X}, \mathcal{W}\right)-R\right|^{+} \leq \Delta \leq I\left(p_{X}, \mathcal{W}\right)-R$, we have

$$
\begin{aligned}
E_{i}\left(R, p_{X}, \mathcal{W}, \alpha\right) & =E_{\mathrm{sp}}\left(R, p_{X}, \mathcal{W}\right)+\Delta=\alpha \\
E_{\emptyset}^{L}\left(R, p_{X}, \mathcal{W}, \alpha\right) & =E_{\mathrm{sp}}\left(R+\Delta, p_{X}, \mathcal{W}\right) .
\end{aligned}
$$

(ii) The above exponents are also achieved using the penalty function $F(t)=\Delta+\lambda|t|^{+}$with

$$
-E_{\mathrm{sp}}^{\prime}\left(R, p_{X}, \mathcal{W}\right) \leq \lambda \leq \frac{1}{-E_{\mathrm{sp}}^{\prime}\left(R+\Delta, p_{X}, \mathcal{W}\right)} .
$$

(iii) If $R \leq R_{c r}\left(p_{X}, \mathcal{W}\right)$ and $0 \leq \Delta \leq R^{\operatorname{conj}}\left(p_{X}, \mathcal{W}\right)-R$, we have

$$
\begin{aligned}
E_{i}\left(R, p_{X}, \mathcal{W}, \alpha\right)= & E_{\mathrm{sp}}\left(R, p_{X}, \mathcal{W}\right)+\Delta=\alpha \\
E_{\emptyset}^{L}\left(R, p_{X}, \mathcal{W}, \alpha\right)= & E_{\mathrm{sp}}\left(R_{2}(\Delta), p_{X}, \mathcal{W}\right) \\
& +F_{R, p_{X}, \mathcal{W}}^{-1}\left(R_{1}(\Delta)-R\right) .
\end{aligned}
$$


(iv) If $R \leq R_{c r}\left(p_{X}, \mathcal{W}\right)$ and $R_{\infty}\left(p_{X}, \mathcal{W}\right)-R \leq \Delta \leq 0$, we have

$$
\begin{aligned}
E_{i}\left(R, p_{X}, \mathcal{W}, \alpha\right)= & E_{\mathrm{sp}}\left(R, p_{X}, \mathcal{W}\right)+\Delta=\alpha \\
E_{\emptyset}^{L}\left(R, p_{X}, \mathcal{W}, \alpha\right)= & E_{\mathrm{sp}}\left(R_{1}(\Delta), p_{X}, \mathcal{W}\right) \\
& +F_{R, p_{X}, \mathcal{W}}^{-1}\left(R_{2}(\Delta)-R\right) .
\end{aligned}
$$

Part (ii) of the proposition implies that not only is the optimal $F$ nonunique under the combinations of $\left(R, p_{X}, \mathcal{W}, \alpha\right)$ of Part (i), but also the Csiszár-Körner rule (2.10) is optimal for any $(\Delta, \lambda)$ in a certain range of values.

Also, while Proposition 5.3 provides simple expressions for the worst case error exponents over $\mathcal{W}$, the exponents for any specific channel $p_{Y \mid X} \in \mathcal{W}$ are obtained by substituting the function (5.12) and its inverse, respectively, into the minimization problem of (4.7). This problem does generally not admit a simple expression.

This leads us back to the question asked at the end of Section II, namely, when does the decoder pay no penalty for not knowing $p_{Y \mid X}$ ? Defining

$$
\underline{E}_{\mathrm{sp}}^{\prime}\left(R, p_{X}, \mathcal{W}\right) \triangleq \min _{p_{Y \mid X} \in \mathcal{W}} E_{\mathrm{sp}}^{\prime}\left(R, p_{X}, p_{Y \mid X}\right)
$$

and

$$
\bar{R}^{\mathrm{conj}}\left(p_{X}, \mathcal{W}\right) \triangleq \max _{p_{Y \mid X} \in \mathcal{W}} R^{\operatorname{conj}}\left(p_{X}, p_{Y \mid X}\right)
$$

we have the following lemma, whose proof appears in Appendix E.

Lemma 5.4:

$$
\begin{aligned}
E_{\mathrm{sp}}^{\prime}\left(R, p_{X}, \mathcal{W}\right) & \geq \underline{E}_{\mathrm{sp}}^{\prime}\left(R, p_{X}, \mathcal{W}\right) \\
\bar{R}^{\mathrm{conj}}\left(p_{X}, \mathcal{W}\right) & \geq R^{\mathrm{conj}}\left(p_{X}, \mathcal{W}\right)
\end{aligned}
$$

with equality if the same $p_{Y \mid X}$ minimizes $E_{\mathrm{sp}}\left(R, p_{X}, p_{Y \mid X}\right)$ at all rates.

Proposition 5.5: Assume that $R, p_{X}, \mathcal{W}, \Delta$, and $\lambda$ are such that

$$
\begin{aligned}
\left|\bar{R}^{\mathrm{conj}}\left(p_{X}, \mathcal{W}\right)-R\right|^{+} & \leq \Delta \leq I\left(p_{X}, \mathcal{W}\right)-R \\
-\underline{E}_{\mathrm{sp}}^{\prime}\left(R, p_{X}, \mathcal{W}\right) & \leq \lambda \leq \frac{1}{-\underline{E}_{\mathrm{sp}}^{\prime}\left(R+\Delta, p_{X}, \mathcal{W}\right)} .
\end{aligned}
$$

Then the pair of incorrect-message and erasure exponents

$$
\left\{E_{\mathrm{sp}}\left(R, p_{X}, p_{Y \mid X}\right)+\Delta, E_{\mathrm{sp}}\left(R+\Delta, p_{X}, p_{Y \mid X}\right)\right\}
$$

is universally attainable over $p_{Y \mid X} \in \mathcal{W}$ using the penalty function $F(t)=\Delta+\lambda|t|^{+}$, and equality holds in the erasure-exponent game of (3.11).

Proof: From (5.19) and (5.22), we have

$$
\begin{aligned}
\left|R^{\operatorname{conj}}\left(p_{X}, p_{Y \mid X}\right)-R\right|^{+} \leq \Delta \leq I\left(p_{X}, p_{Y \mid X}\right) & -R, \\
& \forall p_{Y \mid X} \in \mathcal{W} .
\end{aligned}
$$

Similarly, from (5.18) and (5.23), we have

$$
\begin{aligned}
-E_{\mathrm{sp}}^{\prime}\left(R, p_{X}, p_{Y \mid X}\right) \leq \lambda \leq \frac{1}{-E_{\mathrm{sp}}^{\prime}\left(R+\Delta, p_{X}, p_{Y \mid X}\right)}, & \\
\forall p_{Y \mid X} & \in \mathcal{W} .
\end{aligned}
$$

Then applying Proposition 5.3(ii) with the singleton $\left\{p_{Y \mid X}\right\}$ in place of $\mathcal{W}$ proves the claim.

The set of $(\Delta, \lambda)$ defined by (5.22), (5.23) is smaller than that of Proposition 5.3(i) but is not empty because $\underline{E}_{\mathrm{sp}}^{\prime}\left(R+\Delta, p_{X}, \mathcal{W}\right)$ tends to zero as $\Delta$ approaches the upper limit $I\left(p_{X}, \mathcal{W}\right)-R$. Thus, the universal exponents in (5.24) hold at least in the small erasure-exponent regime (where $\left.E_{\mathrm{sp}}\left(R+\Delta, p_{X}, p_{Y \mid X}\right) \rightarrow 0\right)$ and coincide with those derived by Forney [3, Theorem 3(a)] for symmetric channels, using maximum a posteriori probability (MAP) decoding. For symmetric channels, the same input distribution $p_{X}$ is optimal at all rates. ${ }^{3}$ Our rates are identical to his, i.e., the same optimal error exponents are achieved without knowledge of the channel.

\section{RELATIVE MiNIMAX}

When the compound class $\mathcal{W}$ is so large that $I\left(p_{X}, \mathcal{W}\right) \leq R$, we have seen from Corollary 5.2 that the simple thresholding rule $F(t) \equiv \Delta$ is optimal. Even if $I\left(p_{X}, \mathcal{W}\right)>R$, our minimax criterion (which seeks the worst case error exponents over the class $\mathcal{W}$ ) for designing $F$ might be a pessimistic one. This drawback can be alleviated to some extent using a relative minimax principle, see [12] and references therein. Our proposed approach is to define two functionals $\alpha\left(p_{Y \mid X}\right)$ and $\beta\left(p_{Y \mid X}\right)$ and the relative error exponents

$$
\begin{aligned}
& \Delta_{\alpha} E_{i}\left(R, p_{X}, p_{Y \mid X}, F\right) \triangleq E_{i}\left(R, p_{X}, p_{Y \mid X}, F\right)-\alpha\left(p_{Y \mid X}\right) \\
& \Delta_{\beta} E_{\emptyset}\left(R, p_{X}, p_{Y \mid X}, F\right) \triangleq E_{\emptyset}\left(R, p_{X}, p_{Y \mid X}, F\right)-\beta\left(p_{Y \mid X}\right) .
\end{aligned}
$$

Then solve the constrained optimization problem of (3.9) with the above functionals in place of $E_{i}\left(R, p_{X}, p_{Y \mid X}, F\right)-\alpha$ and $E_{\emptyset}\left(R, p_{X}, p_{Y \mid X}, F\right)$. It is reasonable to choose $\alpha\left(p_{Y \mid X}\right)$ and $\beta\left(p_{Y \mid X}\right)$ large for "good channels" and small for very noisy channels. Also, while $\alpha\left(p_{Y \mid X}\right)$ and $\beta\left(p_{Y \mid X}\right)$ could be the error exponents associated with some reference test, this is not a requirement. A possible choice is

$$
\begin{aligned}
& \alpha\left(p_{Y \mid X}\right)=\Delta \\
& \beta\left(p_{Y \mid X}\right)=E_{\mathrm{sp}}\left(R+\Delta, p_{X}, p_{Y \mid X}\right)
\end{aligned}
$$

which are the error exponents (5.2) and (5.3) corresponding to the thresholding rule $F(t) \equiv \Delta$. Another choice is

$$
\begin{aligned}
& \alpha\left(p_{Y \mid X}\right)=E_{\mathrm{sp}}\left(R, p_{X}, p_{Y \mid X}\right)+\Delta \\
& \beta\left(p_{Y \mid X}\right)=E_{\mathrm{sp}}\left(R+\Delta, p_{X}, p_{Y \mid X}\right)
\end{aligned}
$$

which are the "ideal" Forney exponents—achievable under the assumptions of Proposition 5.5(i).

The relative minimax problem is a simple extension of the minimax problem solved earlier. Define the following functions:

$$
\begin{aligned}
\Delta_{\alpha} E_{r, F}\left(R, p_{X}, \mathcal{W}\right)= & \min _{p_{Y \mid X} \in \mathcal{W}}\left[E_{r, F}\left(R, p_{X}, p_{Y \mid X}\right)\right. \\
& \left.-\alpha\left(p_{Y \mid X}\right)\right], \\
\Delta_{\alpha} E_{\mathrm{sp}}\left(R, p_{X}, \mathcal{W}\right)= & \min _{p_{Y \mid X} \in \mathcal{W}}\left[E_{\mathrm{sp}}\left(R, p_{X}, p_{Y \mid X}\right)\right. \\
& \left.-\alpha\left(p_{Y \mid X}\right)\right], \\
F_{R, p_{X}, \mathcal{W}, \alpha}(t)= & \Delta_{\alpha} E_{\mathrm{sp}}\left(R, p_{X}, \mathcal{W}\right) \\
& -\Delta_{\alpha} E_{\mathrm{sp}}\left(R+t, p_{X}, \mathcal{W}\right) .
\end{aligned}
$$

${ }^{3}$ Forney also studied the case $E_{\emptyset}(R)>E_{i}(R)$, which is not covered by our analysis. 
The function $F_{R, p_{X}, \mathcal{W}, \alpha}(t)$ of (6.5) is increasing and satisfies $F_{R, p_{X}, \mathcal{W}, \alpha}(0)=0$. The above functions $\Delta_{\alpha} E_{r, F}$ and $\Delta_{\alpha} E_{\mathrm{sp}}$ satisfy the following relationship:

$$
\begin{aligned}
& \Delta_{\alpha} E_{r, F}\left(R, p_{X}, \mathcal{W}\right) \\
& \stackrel{\text { (a) }}{=} \min _{p_{Y \mid X} \in \mathcal{W}}\left\{\operatorname { m i n } _ { R ^ { \prime } } \left[E_{\mathrm{sp}}\left(R^{\prime}, p_{X}, p_{Y \mid X}\right)\right.\right. \\
& \left.\left.+F\left(R^{\prime}-R\right)\right]-\alpha\left(p_{Y \mid X}\right)\right\} \\
& =\min _{R^{\prime}}\left\{\operatorname { m i n } _ { p _ { Y | X } \in \mathcal { W } } \left[E_{\mathrm{sp}}\left(R^{\prime}, p_{X}, p_{Y \mid X}\right)\right.\right. \\
& \left.\left.\left.-\alpha\left(p_{Y \mid X}\right)\right]+F\left(R^{\prime}-R\right)\right]\right\} \\
& \stackrel{(\mathrm{b})}{=} \min _{R^{\prime}}\left[\Delta_{\alpha} E_{\mathrm{sp}}\left(R^{\prime}, p_{X}, \mathcal{W}\right)+F\left(R^{\prime}-R\right)\right]
\end{aligned}
$$

where (a) is obtained from (4.7) and (6.3), and (b) from (6.4). Equation (6.6) is of the same form as (4.7), with $\Delta_{\alpha} E_{r, F}$ and $\Delta_{\alpha} E_{\mathrm{sp}}$ in place of $E_{r, F}-\alpha$ and $E_{\mathrm{sp}}-\alpha$, respectively.

Analogously to (5.4)-(5.6), the relative minimax for variablesize decoders is given by

$$
\begin{aligned}
& \Delta_{\beta} E_{\emptyset}^{L}(R, \mathcal{W}, \alpha) \\
& \quad=\max _{p_{X}} \sup _{F \in \mathcal{F}^{L}\left(R, p_{X}, \mathcal{W}, \alpha\right)} \Delta_{\beta} E_{r,\left|F^{-1}\right|^{+}}\left(R, p_{X}, \mathcal{W}\right)
\end{aligned}
$$

where the feasible set $\mathcal{F}^{L}\left(R, p_{X}, \mathcal{W}, \alpha\right)$ is the set of functions $F$ that satisfy

$$
\Delta_{\alpha} E_{r, F}\left(R, p_{X}, \mathcal{W}\right) \geq 0
$$

as well as the previous continuity and monotonicity conditions. The following proposition is analogous to Proposition 5.1.

Proposition 6.1: The supremum over $F$ in (6.7) is achieved by

$$
\begin{aligned}
F^{L *}(t) & =F_{R, p_{X}, \mathcal{W}, \alpha}(t)-\Delta_{\alpha} E_{\mathrm{sp}}\left(R, p_{X}, \mathcal{W}\right) \\
& =-\Delta_{\alpha} E_{\mathrm{sp}}\left(R+t, p_{X}, \mathcal{W}\right)
\end{aligned}
$$

independently of the choice of $\beta$. The relative minimax is given by

$$
\Delta_{\beta} E_{\emptyset}^{L}(R, \mathcal{W}, \alpha)=\max _{p_{X}} \Delta_{\beta} E_{r,\left|\left(F^{L *}\right)^{-1}\right|}\left(R, p_{X}, \mathcal{W}\right) .
$$

Proof: The proof exploits the same monotonicity property $\left(E_{r, F} \leq E_{r, G}\right.$ for $F \preceq G$ ) that was used to derive the optimal $F$ in (5.12). The supremum over $F$ is obtained by following the steps of the proof of Proposition 5.1, substituting $\Delta_{\alpha} E_{r, F}$, $\Delta_{\alpha} E_{\mathrm{sp}}$, and $F_{R, p_{X}, \mathcal{W}, \alpha}$ for $E_{r, F}-\alpha, E_{\mathrm{sp}}-\alpha$, and $F_{R, p_{X}, \mathcal{W}}$, respectively. The relative minimax is obtained by substituting the optimal $F$ into (6.7).

We would like to know how much influence the reference function $\alpha$ has on the optimal $F$. For the "Forney reference exponent function" $\alpha$ of (6.1), we obtain the optimal $F$ from (6.8) and (6.4):

$$
\begin{gathered}
F^{L *}(t)=-\min _{p_{Y \mid X} \in \mathcal{W}}\left[E_{\mathrm{sp}}\left(R+t, p_{X}, p_{Y \mid X}\right)-\alpha\left(p_{Y \mid X}\right)\right] \\
=-\min _{p_{Y \mid X} \in \mathcal{W}}\left[E_{\mathrm{sp}}\left(R+t, p_{X}, p_{Y \mid X}\right)\right. \\
\left.-E_{\mathrm{sp}}\left(R, p_{X}, p_{Y \mid X}\right)-\Delta\right]
\end{gathered}
$$

$$
\begin{gathered}
=\Delta+\max _{p_{Y \mid X} \in \mathcal{W}}\left[E_{\mathrm{sp}}\left(R, p_{X}, p_{Y \mid X}\right)\right. \\
\left.\quad-E_{\mathrm{sp}}\left(R+t, p_{X}, p_{Y \mid X}\right)\right] \\
=\Delta+\max _{p_{Y \mid X} \in \mathcal{W}} F_{R, p_{X}, p_{Y \mid X}}(t) .
\end{gathered}
$$

Interestingly, the maximum above is often achieved by the cleanest channel in $\mathcal{W}$-for which $E_{\mathrm{sp}}\left(R, p_{X}, p_{Y \mid X}\right)$ is large and $E_{\mathrm{sp}}\left(R+t, p_{X}, p_{Y \mid X}\right)$ falls off rapidly as $t$ increases. This stands in contrast to (5.12) which may be written as

$$
\begin{aligned}
F^{L *}(t)= & \alpha-\min _{p_{Y \mid X} \in \mathcal{W}} E_{\mathrm{sp}}\left(R+t, p_{X}, p_{Y \mid X}\right) \\
= & \Delta+\min _{p_{Y \mid X} \in \mathcal{W}} E_{\mathrm{sp}}\left(R, p_{X}, p_{Y \mid X}\right) \\
& -\min _{p_{Y \mid X} \in \mathcal{W}} E_{\mathrm{sp}}\left(R+t, p_{X}, p_{Y \mid X}\right) .
\end{aligned}
$$

In (6.10), the minima are achieved by the noisiest channel at rates $R$ and $R+t$, respectively. Also note that $F^{L *}(t)$ from (6.9) is uniformly larger than $F^{L *}(t)$ from (6.10) and thus results in larger incorrect-message exponents.

For $R>I\left(p_{X}, \mathcal{W}\right)$, Corollary 5.2 has shown that the minimax criterion is maximized by the thresholding rule $F(t)=\Delta$ which yields

$$
\begin{aligned}
& E_{i}\left(R, p_{X}, p_{Y \mid X}\right)=\Delta \\
& E_{\emptyset}\left(R, p_{X}, p_{Y \mid X}\right)=E_{\mathrm{sp}}\left(R+\Delta, p_{X}, p_{Y \mid X}\right), \quad \forall p_{Y \mid X} .
\end{aligned}
$$

The relative minimax criterion based on $\alpha\left(p_{Y \mid X}\right)$ of (6.1) yields a higher $E_{i}\left(R, p_{X}, p_{Y \mid X}\right)$ for good channels and this is counterbalanced by a lower $E_{\emptyset}\left(R, p_{X}, p_{Y \mid X}\right)$. Thus, the primary advantage of the relative minimax approach is that $\alpha\left(p_{Y \mid X}\right)$ can be chosen to more finely balance the error exponents across the range of channels of interest.

\section{COMPOUND BINARY-SYMMETRIC CHANNEL}

We have evaluated the incorrect-message and erasure exponents of (5.24) for the compound BSC with crossover probability $\rho \in\left[\rho_{\min }, \rho_{\max }\right]$, where $0<\rho_{\min }<\rho_{\max } \leq \frac{1}{2}$. The class $\mathcal{W}$ may be identified with the interval $\left[\rho_{\min }, \rho_{\max }\right]$, where $\rho_{\min }$ and $\rho_{\max }$ correspond to the cleanest and noisiest channels in $\mathcal{W}$, respectively. Denote by $h_{2}(\rho) \triangleq-\rho \log \rho-(1-\rho) \log (1-\rho)$ the binary entropy function, by $h_{2}^{-1}(\cdot)$ the inverse of that function over the range $\left[0, \frac{1}{2}\right]$, and by $p_{\rho}$ the Bernoulli pmf with parameter $\rho$.

Capacity of the BSC is given by $C(\rho)=1-h_{2}(\rho)$, and the sphere packing exponent by [1, p. 195]

$$
\begin{aligned}
E_{\mathrm{sp}}(R, \rho) & =D\left(p_{\rho_{R}} \| p_{\rho}\right) \\
& =\rho_{R} \log \frac{\rho_{R}}{\rho}+\left(1-\rho_{R}\right) \log \frac{1-\rho_{R}}{1-\rho}, \\
& 0 \leq R \leq C(\rho)
\end{aligned}
$$

where $\rho_{R}=h_{2}^{-1}(1-R) \geq \rho$. The optimal input distribution $p_{X}$ is uniform at all rates and will be omitted from the list of arguments of the functions $E_{r, F}, E_{\mathrm{sp}}$, and $F_{R}$ below. The critical rate is

$$
R_{c r}(\rho)=1-h_{2}\left(\frac{1}{1+\sqrt{1 / \rho^{2}-1}}\right)
$$

and $E_{\mathrm{sp}}(0, \rho)=-\log \sqrt{4 \rho(1-\rho)}$. 
The capacity and sphere-packing exponent for the compound BSC are respectively given by $C(\mathcal{W})=C\left(\rho_{\max }\right)$ and

$$
\begin{aligned}
E_{\mathrm{sp}}(R, \mathcal{W}) & =\min _{\rho_{\min } \leq \rho \leq \rho_{\max }} E_{\mathrm{sp}}(R, \rho) \\
& =E_{\mathrm{sp}}\left(R, \rho_{\max }\right) .
\end{aligned}
$$

For $R \geq C(\mathcal{W})$, the optimal $F$ is the thresholding rule of (5.1), and (5.2), (5.3) yield

$$
E_{i}(R, \rho)=\Delta \quad \text { and } \quad E_{\emptyset}(R, \rho)=E_{\mathrm{sp}}(R+\Delta, \rho) .
$$

In the remainder of this section, we assume $R<C(\mathcal{W})$, in which case $E_{\mathrm{sp}}(R, \mathcal{W})>0$.

Optimal F: For any $0 \leq \Delta \leq C(\rho)-R$, we have $\rho \leq$ $\rho_{R+\Delta} \leq \rho_{R} \leq \frac{1}{2}$. From (7.1) we have

$$
\begin{aligned}
F_{R, \rho}(t)= & E_{\mathrm{sp}}(R, \rho)-E_{\mathrm{sp}}(R+t, \rho) \\
= & D\left(p_{\rho_{R}} \| p_{\rho}\right)-D\left(p_{\rho_{R+t}} \| p_{\rho}\right) \\
= & h_{2}\left(\rho_{R}\right)-h_{2}\left(\rho_{R+t}\right)+\left(\rho_{R}-\rho_{R+t}\right) \\
& \times\left(\log \frac{1}{\rho}-\log \frac{1}{1-\rho}\right) \\
= & h_{2}\left(\rho_{R}\right)-h_{2}\left(\rho_{R+t}\right)+\left(\rho_{R}-\rho_{R+t}\right) \\
& \times \log \left(\frac{1}{\rho}-1\right), \quad t \geq 0
\end{aligned}
$$

which is a decreasing function of $\rho$.

Evaluating the optimal $F$ from (5.12), we have

$$
\begin{aligned}
F^{L *}(t) & =\Delta+F_{R, \mathcal{W}}(t) \\
& =\Delta+E_{\mathrm{sp}}(R, \mathcal{W})-E_{\mathrm{sp}}(R+t, \mathcal{W}) \\
& =\Delta+E_{\mathrm{sp}}\left(R, \rho_{\max }\right)-E_{\mathrm{sp}}\left(R+t, \rho_{\max }\right) \\
& =\Delta+F_{R, \rho_{\max }}(t), \quad t \geq 0 .
\end{aligned}
$$

Observe that the optimal $F$ is determined by the noisiest channel $\left(\rho_{\max }\right)$ and does not depend at all on $\rho_{\min }$.

This contrasts with the relative minimax criterion with $\alpha\left(p_{Y \mid X}\right)$ of (6.1), where evaluation of the optimal $F$ from (6.9) yields

$$
\begin{aligned}
F^{L *}(t) & =\Delta+\max _{\rho_{\min } \leq \rho \leq \rho_{\max }} F_{R, \rho}(t) \\
& =\Delta+F_{R, \rho_{\min }}(t), \quad t \geq 0
\end{aligned}
$$

which is determined by the cleanest channel $\left(\rho_{\min }\right)$ and does not depend on $\rho_{\max }$.

Optimal Error Exponents: Fig. 2 displays $E_{\emptyset}(R, \mathcal{W})$ and $E_{i}(R, \mathcal{W})$ when $\rho_{\max }=0.1$ (the value of $\rho_{\min }$ is immaterial for the minimax criterion here), $R=0.1<C\left(\rho_{\max }\right) \approx$ 0.53 , and the exponent tradeoff parameter $\Delta$ ranges from $-R$ to $C\left(\rho_{\max }\right)-R$. The resulting exponents are obtained from Proposition 5.3, and the existence of the three regimes, respectively described by (5.14), (5.16), and (5.17), may be seen in the figure.

Next we derive expressions for $E_{\mathrm{sp}}^{\prime}(R, \mathcal{W})$ and $\underline{E}_{\mathrm{sp}}^{\prime}(R, \mathcal{W})$. The derivations are simplified if instead of working with the crossover probability $\rho$, we use the following reparameterization:

$$
\mu \triangleq \rho^{-1}-1, \quad \mu_{\max } \triangleq \rho_{\min }^{-1}-1, \quad \mu_{\min } \triangleq \rho_{\max }^{-1}-1
$$

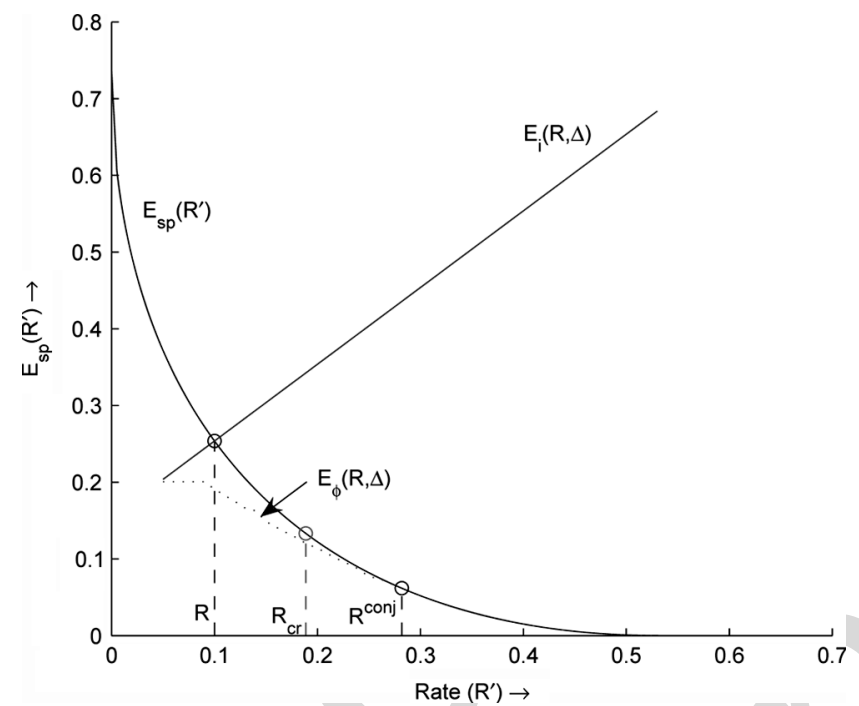

Fig. 2. Erasure and incorrect-message exponents $E_{\emptyset}(R, \mathcal{W})$ and $E_{i}(R, \mathcal{W})$ when $R=0.1$ and $\mathcal{W}$ is the family of BSCs with crossover probability $\rho \leq$ $\rho_{\max }=0.1$. The compound channel capacity is $C\left(\rho_{\max }\right) \approx 0.53$.

and

$\mu_{R} \triangleq \rho_{R}^{-1}-1=\frac{1}{h_{2}^{-1}(1-R)}-1 \Leftrightarrow R=1-h_{2}\left(\frac{1}{1+\mu}\right)$

where $\mu_{R}$ increases monotonically from 1 to $\infty$ as $R$ increases from 0 to 1 . With this notation, we have $\rho=\frac{1}{1+\mu}$ and $\mu_{\max } \geq$ $\mu \geq \mu_{R+\Delta} \geq \mu_{R} \geq 1$. Also

$$
\begin{aligned}
\frac{d R}{d \rho_{R}} & =-\frac{d h_{2}\left(\rho_{R}\right)}{d \rho_{R}}=-\frac{\log \mu_{R}}{\ln 2} \\
\frac{d E_{\mathrm{sp}}(R, \rho)}{d \rho_{R}} & =\frac{\log \mu-\log \mu_{R}}{\ln 2}
\end{aligned}
$$

$-E_{\mathrm{sp}}^{\prime}(R, \rho)=\frac{d E_{\mathrm{sp}}(R, \rho) / d \rho_{R}}{-d R / d \rho_{R}}=\frac{\log \mu}{\log \mu_{R}}-1=\frac{\log \mu / \mu_{R}}{\log \mu_{R}}$.

From (5.18) and (7.3), we obtain

$$
-\underline{E}_{\mathrm{sp}}^{\prime}(R, \mathcal{W})=-\min _{\rho_{\min } \leq \rho \leq \rho_{\max }} E_{\mathrm{sp}}^{\prime}(R, \rho)=\frac{\log \mu_{\max } / \mu_{R}}{\log \mu_{R}} .
$$

Observe that the minimizing $\rho$ is $\rho_{\min }$, i.e., the cleanest channel in $\mathcal{W}$. In contrast, from (7.2), we have

$$
\begin{aligned}
-E_{\mathrm{sp}}^{\prime}(R, \mathcal{W}) & =-E_{\mathrm{sp}}^{\prime}\left(R, \rho_{\max }\right) \\
& =\frac{\log \mu_{\min } / \mu_{R}}{\log \mu_{R}}<-\underline{E}_{\mathrm{sp}}^{\prime}(R, \mathcal{W})
\end{aligned}
$$

which is determined by the noisiest channel $\left(\rho_{\max }\right)$.

Conditions for Universality: Next we evaluate $\bar{R}^{\text {conj }}(\mathcal{W})$ from (5.19). For a given $\rho$, the conjugate rate of $R$ is obtained from (7.3)

$$
\begin{aligned}
& -E_{\mathrm{sp}}^{\prime}(R, \rho)=\frac{1}{-E_{\mathrm{sp}}^{\prime}\left(R^{\mathrm{conj}}, \rho\right)} \\
& \frac{\log \left(\mu / \mu_{R}\right)}{\log \mu_{R}}=\frac{\log \mu_{R^{\mathrm{conj}}}}{\log \left(\mu / \mu_{\left.R^{\mathrm{conj}}\right)}\right.}
\end{aligned}
$$


hence

$$
\begin{aligned}
& \mu_{R^{\mathrm{conj}}}=\frac{\mu}{\mu_{R}} \\
& R^{\mathrm{conj}}(\mu)=1-h_{2}\left(\frac{1}{1+\mu / \mu_{R}}\right) \\
& \bar{R}^{\text {conj }}(\mathcal{W})=\max _{\mu_{\min } \leq \mu \leq \mu_{\max }} R^{\text {conj }}(\mu) \\
& =R^{\mathrm{conj}}\left(\mu_{\max }\right) \text {. }
\end{aligned}
$$

From (5.10) and (7.2), we have

$$
R^{\mathrm{conj}}(\mathcal{W})=R^{\mathrm{conj}}\left(\mu_{\min }\right) .
$$

Analogously to (7.5), observe that both $\bar{R}^{\text {conj }}(\mathcal{W})$ and $R^{\operatorname{conj}}(\mathcal{W})$ are determined by the cleanest and noisiest channels in $\mathcal{W}$, respectively.

We can now evaluate the conditions of Proposition 5.5, under which $F(t)=\Delta+\lambda|t|^{+}$is universal (subject to conditions on $\Delta$ and $\lambda$ ). In (5.22), $\Delta$ must satisfy (7.6) shown at the bottom of the page. The left side is zero if $\mu_{\max } \leq \mu_{R}^{2}$. If $\mu_{\max }>\mu_{R}^{2}$, the argument of $|\cdot|^{+}$is positive, and we need $\mu_{\max } / \mu_{R}<\mu_{\min }$ to ensure that the left side is lower than the right side. Hence, there exists a nonempty range of values of $\Delta$ satisfying (7.6) if and only if

$$
\mu_{R} \geq \min \left\{\sqrt{\mu_{\max }}, \frac{\mu_{\max }}{\mu_{\min }}\right\}
$$

which may also be written as

$$
\mu_{\max } \leq \max \left\{\mu_{R}^{2}, \mu_{R} \mu_{\min }\right\} .
$$

Next, substituting (7.4) into (5.23), we obtain the following condition for $\lambda$ :

$$
\frac{\log \mu_{\max } / \mu_{R}}{\log \mu_{R}} \leq \lambda \leq \frac{\log \mu_{R+\Delta}}{\log \mu_{\max } / \mu_{R+\Delta}} .
$$

This equation has a solution if and only if the left side does not exceed the right side, i.e.,

$$
\mu_{\max } \leq \mu_{R} \mu_{R+\Delta}
$$

or, equivalently, $\rho_{\min } \geq\left(1+\mu_{R} \mu_{R+\Delta}\right)^{-1}$. Since $\mu_{R}$ is an increasing function of $R$, the larger the values of $R$ and $\Delta$, the lower the value of $\rho_{\min }$ for which the universality property still holds.

If equality holds in (7.8), the only feasible value of $\lambda$ is

$$
\lambda=\frac{\log \mu_{R+\Delta}}{\log \mu_{R}} \geq 1 \text {. }
$$

This value of $\lambda$ remains feasible if (7.8) holds with strict inequality.

\section{DISCUSSION}

The $\mathcal{F}$-MMI decision rule of (3.1) is a generalization of Csiszár and Körner's MMI decoder with erasure option. The weighting function $F$ in (3.1) can be optimized in an asymptotic Neyman-Pearson sense given a compound class of channels $\mathcal{W}$. An explicit formula has been derived in terms of the sphere-packing exponent function for $F$ that maximizes the erasure exponent subject to a constraint on the incorrect-message exponent. The optimal $F$ is generally nonunique but agrees with existing designs in special cases of interest.

In particular, Corollary 5.2 shows that the simple thresholding rule $F(t) \equiv \Delta$ is optimal if $R \geq I\left(p_{X}, \mathcal{W}\right)$, i.e., when the transmission rate cannot be reliably supported by the worst channel in $\mathcal{W}$. When $R<I\left(p_{X}, \mathcal{W}\right)$, Proposition 5.5 shows that for small erasure exponents, our expressions for the optimal exponents coincide with those derived by Forney [3] for symmetric channels, where the same input distribution $p_{X}$ is optimal at all rates. In this regime, Csiszár and Körner's rule $F(t)=$ $\Delta+\lambda|t|^{+}$is also universal under some conditions on the parameter pair $(\Delta, \lambda)$. It is also worth noting that while suboptimal, the design $F(t)=\Delta+t$ yields an empirical version of Forney's simple decision rule (2.5).

Previous work [6] using a different universal decoder had shown that Forney's exponents can be matched in the special case where undetected-error and erasure exponents are equal (corresponding to $T=0$ in Forney's rule (2.4)). Our results show that this property extends beyond this special case, albeit not everywhere.

Another analogy between Forney's suboptimal decision rule (2.5) and ours (3.1) is that the former is based on the likelihood score of each candidate message and its highest scoring competitor, and ours uses empirical mutual information instead of likelihood. Our results imply that (2.5) is optimal (in terms of error exponents) in the special regime identified above.

The relative minimax criterion of Section VI is attractive when the compound class $\mathcal{W}$ is broad (or difficult to pick) as it allows finer tuning of the error exponents for different channels in $\mathcal{W}$. The class $\mathcal{W}$ could conceivably be as large as $\mathcal{P}_{Y \mid X}$, the set of all DMCs. Depending on the choice of the reference exponents and the class $\mathcal{W}$, the design of $F$ may be determined by the least noisy channel in $\mathcal{W}$ instead of the noisiest one.

Finally, we have extended our framework to decoding for compound MACs. Those results will be presented elsewhere.

$$
\begin{gathered}
\left|\bar{R}^{\mathrm{conj}}(\mathcal{W})-R\right|^{+} \leq \Delta \leq C(\mathcal{W})-R \\
\mid R^{\text {conj }\left(\mu_{\max }\right)-\left.R\right|^{+}} \leq \Delta \leq C\left(\mu_{\min }\right)-R \\
\left|h_{2}\left(\frac{1}{1+\mu_{R}}\right)-h_{2}\left(\frac{1}{1+\mu_{\max } / \mu_{R}}\right)\right|^{+} \leq \Delta \leq h_{2}\left(\frac{1}{1+\mu_{R}}\right)-h_{2}\left(\frac{1}{1+\mu_{\min }}\right) .
\end{gathered}
$$




\section{APPENDIX A \\ PROOF OF PROPOSITION 4.1}

(i) follows from (4.3), restated below

$$
\begin{aligned}
E_{r, F}\left(R, p_{X}, p_{Y \mid X}\right)=\min _{\tilde{p}_{Y \mid X}}[ & D\left(\tilde{p}_{Y \mid X} \| p_{Y \mid X} \mid p_{X}\right) \\
& \left.+F\left(I\left(p_{X}, \tilde{p}_{Y \mid X}\right)-R\right)\right]
\end{aligned}
$$

and the fact that $F$ is increasing. Indeed, let $\tilde{p}_{Y \mid X}^{[R]}$ achieve the minimum of (A.1), at rate $R$, and choose $R<R^{\prime} \leq$ $I\left(p_{X}, p_{Y \mid X}\right)$. Then evaluating (A.1) at $R^{\prime}$ we have

$$
\begin{aligned}
E_{r, F} & \left(R^{\prime}, p_{X}, p_{Y \mid X}\right) \\
& \leq D\left(\tilde{p}_{Y \mid X}^{[R]} \| p_{Y \mid X} \mid p_{X}\right)+F\left(I\left(p_{X}, \tilde{p}_{Y \mid X}^{[R]}\right)-R^{\prime}\right) \\
& <D\left(\tilde{p}_{Y \mid X}^{[R]} \| p_{Y \mid X} \mid p_{X}\right)+F\left(I\left(p_{X}, \tilde{p}_{Y \mid X}^{[R]}\right)-R\right) \\
& =\min _{\tilde{p}_{Y \mid X}}\left[D\left(\tilde{p}_{Y \mid X} \| p_{Y \mid X} \mid p_{X}\right)+F\left(I\left(p_{X}, \tilde{p}_{Y \mid X}\right)-R\right)\right] \\
& =E_{r, F}\left(R, p_{X}, p_{Y \mid X}\right) .
\end{aligned}
$$

(ii) holds for the same reason as above.

(iii) Since the function $E_{\mathrm{sp}}\left(R, p_{X}, p_{Y \mid X}\right)$ is decreasing in $R$ for $R \leq I\left(p_{X}, p_{Y \mid X}\right)$, it follows from (4.1) that

$$
\begin{array}{r}
E_{\mathrm{Sp}}\left(R, p_{X}, p_{Y \mid X}\right)=\min _{\tilde{p}_{Y \mid X}: I\left(p_{X}, \tilde{p}_{Y \mid X}\right)=R} D\left(\tilde{p}_{Y \mid X} \| p_{Y \mid X} \mid p_{X}\right), \\
\forall R \leq I\left(p_{X}, p_{Y \mid X}\right) . \quad \text { (A.2) }
\end{array}
$$

For any $\tilde{p}_{Y \mid X}$ such that $I\left(p_{X}, \tilde{p}_{Y \mid X}\right) \geq I\left(p_{X}, p_{Y \mid X}\right)$, we have $F\left(I\left(p_{X}, \tilde{p}_{Y \mid X}\right)-R\right) \geq F\left(I\left(p_{X}, p_{Y \mid X}\right)-R\right)$ because $F$ is nondecreasing. The minimum of $D+F$ in (A.1) over all such $\tilde{p}_{Y \mid X}$ is therefore achieved by $\tilde{p}_{Y \mid X}=p_{Y \mid X}$. Thus, $\tilde{p}_{Y \mid X}$ that achieves the unconstrained minimum in (A.1) must satisfy $I\left(p_{X}, \tilde{p}_{Y \mid X}\right) \leq I\left(p_{X}, p_{Y \mid X}\right)$. Hence, we have the equation at the bottom of the page, where (a) is due to (A.2), and (b) holds because $E_{\mathrm{sp}}\left(R^{\prime}, p_{X}, p_{Y \mid X}\right)=0$ for $R^{\prime} \geq I\left(p_{X}, p_{Y \mid X}\right)$ and $F$ is nondecreasing.

(iv) The claim follows directly from the definitions (4.2) and (4.4), e.g., (i) is proved by observing that $E_{r, F}\left(R, p_{X}, p_{Y \mid X}\right)<$ $E_{r, F}\left(R^{\prime}, p_{X}, p_{Y \mid X}\right)$ implies

$$
\begin{aligned}
E_{r, F}\left(R, p_{X}, \mathcal{W}\right) & =\min _{p_{Y \mid X} \in \mathcal{W}} E_{r, F}\left(R, p_{X}, p_{Y \mid X}\right) \\
& <\min _{p_{Y \mid X} \in \mathcal{W}} E_{r, F}\left(R^{\prime}, p_{X}, p_{Y \mid X}\right) \\
& =E_{r, F}\left(R^{\prime}, p_{X}, \mathcal{W}\right)
\end{aligned}
$$

for all $R \leq I\left(p_{X}, \mathcal{W}\right)$. Property (ii) is immediate as well. Property (iii) holds because

$$
\begin{aligned}
E_{r, F} & \left(R, p_{X}, \mathcal{W}\right) \\
& =\min _{p_{Y \mid X} \in \mathcal{W}} \min _{R^{\prime}}\left[E_{\mathrm{sp}}\left(R^{\prime}, p_{X}, p_{Y \mid X}\right)+F\left(R^{\prime}-R\right)\right] \\
& =\min _{R^{\prime}}\left[\min _{p_{Y \mid X} \in \mathcal{W}} E_{\mathrm{sp}}\left(R^{\prime}, p_{X}, p_{Y \mid X}\right)+F\left(R^{\prime}-R\right)\right] \\
& =\min _{R^{\prime}}\left[E_{\mathrm{sp}}\left(R^{\prime}, p_{X}, \mathcal{W}\right)+F\left(R^{\prime}-R\right)\right] .
\end{aligned}
$$

APPENDIX B

PROOF OF PROPOSITION 4.2

Given the pmf $p_{X}$, choose any type $p_{\boldsymbol{x}}$ such that $\max _{x \in \mathcal{X}}\left|p_{\boldsymbol{x}}(x)-p_{X}(x)\right| \leq \frac{|\mathcal{X}|}{N} \cdot{ }^{4}$ Define

$$
\begin{aligned}
& E_{r, F, N}\left(R, p_{\boldsymbol{x}}, p_{Y \mid X}\right) \\
& \quad=\min _{p_{\boldsymbol{y} \mid \boldsymbol{x}}}\left[D\left(p_{\boldsymbol{y} \mid \boldsymbol{x}} \| p_{Y \mid X} \mid p_{\boldsymbol{x}}\right)+F(I(\boldsymbol{x} ; \boldsymbol{y})-R)\right]
\end{aligned}
$$

and

$$
E_{s p, N}\left(R, p_{\boldsymbol{x}}, p_{Y \mid X}\right)=\min _{p_{\boldsymbol{y} \mid x}: I(\boldsymbol{x} ; \boldsymbol{y}) \leq R} D\left(p_{\boldsymbol{y} \mid \boldsymbol{x}}|| p_{Y \mid X} \mid p_{\boldsymbol{x}}\right)
$$

which differ from (4.3) and (4.1) in that the minimization is performed over conditional types instead of general conditional pmfs. We have

$$
\begin{aligned}
\lim _{N \rightarrow \infty} E_{r, F, N}\left(R, p_{\boldsymbol{x}}, p_{Y \mid X}\right) & =E_{r, F}\left(R, p_{X}, p_{Y \mid X}\right) \\
\lim _{N \rightarrow \infty} E_{s p, N}\left(R, p_{\boldsymbol{x}}, p_{Y \mid X}\right) & =E_{\mathrm{sp}}\left(R, p_{X}, p_{Y \mid X}\right)
\end{aligned}
$$

by continuity of the divergence functional and of $F$ in the region where the minimand $D+F$ is finite.

We will use the following two standard inequalities.

1) Given an arbitrary sequence $\boldsymbol{y}$, draw $\boldsymbol{x}^{\prime}$ independently of $\boldsymbol{y}$ and uniformly over a fixed type class $T_{\boldsymbol{x}}$. Then [1]

$$
\operatorname{Pr}\left[T_{\boldsymbol{x}^{\prime} \mid \boldsymbol{y}}\right]=\frac{\left|T_{\boldsymbol{x}^{\prime} \mid \boldsymbol{y}}\right|}{\left|T_{\boldsymbol{x}}\right|}=\frac{\left|T_{\boldsymbol{x}^{\prime} \mid \boldsymbol{y}}\right|}{\left|T_{\boldsymbol{x}^{\prime}}\right|} \doteq 2^{-N I\left(\boldsymbol{x}^{\prime} ; \boldsymbol{y}\right)}
$$

Hence, for any $0 \leq \nu \leq H\left(p_{\boldsymbol{x}}\right)$

$$
\operatorname{Pr}\left[I\left(\boldsymbol{x}^{\prime} ; \boldsymbol{y}\right) \geq \nu\right]=\sum_{T_{\boldsymbol{x}^{\prime} \mid \boldsymbol{y}}} \operatorname{Pr}\left[T_{\boldsymbol{x}^{\prime} \mid \boldsymbol{y}}\right] \mathbb{1}_{\left\{I\left(\boldsymbol{x}^{\prime} ; \boldsymbol{y}\right) \geq \nu\right\}}
$$

${ }^{4}$ For instance, truncate each $p_{X}(x)$ down to the nearest integer multiple of $1 / N$ and add $a / N$ to the smallest resulting value to obtain $p_{\boldsymbol{x}}(x), x \in \mathcal{X}$, summing to one. $a$ is an integer in the range $\{0,1, \ldots,|\mathcal{X}|-1\}$.

$$
\begin{aligned}
E_{r, F}\left(R, p_{X}, p_{Y \mid X}\right) & =\cos _{\tilde{p}_{Y \mid X}: I\left(p_{X}, \tilde{p}_{Y \mid X}\right) \leq I\left(p_{X}, p_{Y \mid X}\right)}\left[D\left(\tilde{p}_{Y \mid X} \| p_{Y \mid X} \mid p_{X}\right)+F\left(I\left(p_{X}, \tilde{p}_{Y \mid X}\right)-R\right)\right] \\
& =\min _{R^{\prime} \leq I\left(p_{X}, p_{Y \mid X}\right)} \tilde{p}_{Y \mid X}: I\left(p_{X}, \tilde{p}_{Y \mid X}\right)=R^{\prime} \\
& \stackrel{(\mathrm{a})}{=} \min _{R^{\prime} \leq I\left(p_{X}, p_{Y \mid X}\right)}\left[E_{\mathrm{sp}}\left(R^{\prime}, p_{X}, p_{Y \mid X}\right)+F\left(R^{\prime}-R\right)\right] \\
& \stackrel{(\mathrm{b})}{=} \min _{R^{\prime}}\left[E_{\mathrm{sp}}\left(R^{\prime}, p_{X}, p_{Y \mid X}\right)+F\left(p_{X}\right)+F\left(R^{\prime}-R\right)\right]
\end{aligned}
$$




$$
\begin{aligned}
& \doteq \sum_{T_{\boldsymbol{x}^{\prime} \mid \boldsymbol{y}}} 2^{-N I\left(\boldsymbol{x}^{\prime} ; \boldsymbol{y}\right)} \mathbb{1}_{\left\{I\left(\boldsymbol{x}^{\prime} ; \boldsymbol{y}\right) \geq \nu\right\}} \\
& \stackrel{(\mathrm{a})}{\doteq} \max _{T_{\boldsymbol{x}^{\prime} \mid \boldsymbol{y}}} 2^{-N I\left(\boldsymbol{x}^{\prime} ; \boldsymbol{y}\right)} \mathbb{1}_{\left\{I\left(\boldsymbol{x}^{\prime} ; \boldsymbol{y}\right) \geq \nu\right\}} \\
& \doteq 2^{-N \nu}
\end{aligned}
$$

where (a) holds because the number of types is polynomial in $N$. For $\nu>H\left(p_{\boldsymbol{x}}\right)$ we have $\operatorname{Pr}\left[I\left(\boldsymbol{x}^{\prime} ; \boldsymbol{y}\right) \geq \nu\right]=0$.

2) Given an arbitrary sequence $\boldsymbol{x}$, draw $\boldsymbol{y}$ from the conditional $\operatorname{pmf} p_{Y \mid X}^{N}(\cdot \mid \boldsymbol{x})$. We have [1]

$$
\operatorname{Pr}\left[T_{\boldsymbol{y} \mid \boldsymbol{x}}\right] \doteq 2^{-N D\left(p_{\boldsymbol{y} \mid \boldsymbol{x}} \| p_{Y \mid X} \mid p_{\boldsymbol{x}}\right)} .
$$

Then, for any $\nu>0$

$$
\begin{aligned}
\operatorname{Pr}[I(\boldsymbol{x} ; \boldsymbol{y}) \leq \nu]= & \sum_{T_{\boldsymbol{y} \mid \boldsymbol{x}}} \operatorname{Pr}\left[T_{\boldsymbol{y} \mid \boldsymbol{x}}\right] \\
& \times \mathbb{1}_{\{I(\boldsymbol{x} ; \boldsymbol{y}) \leq \nu\}} \\
\doteq & \sum_{p_{\boldsymbol{y} \mid \boldsymbol{x}}} 2^{-N D\left(p_{\boldsymbol{y} \mid \boldsymbol{x}} \| p_{Y \mid X} \mid p_{\boldsymbol{x}}\right)} \\
& \times \mathbb{1}_{\{I(\boldsymbol{x} ; \boldsymbol{y}) \leq \nu\}} \\
\doteq & \max _{p_{\boldsymbol{y} \mid \boldsymbol{x}}} 2^{-N D\left(p_{\boldsymbol{y} \mid \boldsymbol{x}} \| p_{Y \mid X} \mid p_{\boldsymbol{x}}\right)} \\
& \times \mathbb{1}_{\{I(\boldsymbol{x} ; \boldsymbol{y}) \leq \nu\}} \\
= & \max _{p_{\boldsymbol{y} \mid \boldsymbol{x}}: I(\boldsymbol{x} ; \boldsymbol{y}) \leq \nu} 2^{-N D\left(p_{\boldsymbol{y} \mid \boldsymbol{x}} \| p_{Y \mid X} \mid p_{\boldsymbol{x}}\right)} \\
= & 2^{-N E_{s p, N}\left(\nu, p_{\boldsymbol{x}}, p_{Y \mid X}\right)} .
\end{aligned}
$$

Incorrect Messages: The codewords are drawn independently and uniformly from type class $T_{\boldsymbol{x}}$. Since the conditional error probability is independent of the transmitted message, assume without loss of generality that message $m=1$ was transmitted. An incorrect codeword $\boldsymbol{x}(i)$ appears on the decoder's list if $i>1$ and

$$
I(\boldsymbol{x}(i) ; \boldsymbol{y}) \geq R+\max _{j \neq i} F(I(\boldsymbol{x}(j) ; \boldsymbol{y})-R) .
$$

Let $\boldsymbol{x}=\boldsymbol{x}(1)$. To evaluate the expected number of incorrect codewords on the list, we first fix $\boldsymbol{y}$.

Given $\boldsymbol{y}$, define the i.i.d. random variables $Z_{i}=I(\boldsymbol{x}(i) ; \boldsymbol{y})-$ $R$ for $2 \leq i \leq 2^{N R}$. Also, let $z_{1}=I(\boldsymbol{x} ; \boldsymbol{y})-R$, which is a function of the joint type $p_{x y}$. The expected number of incorrect codewords on the list depends on $(\boldsymbol{x}, \boldsymbol{y})$ only via their joint type and is given by

$$
\begin{aligned}
\mathbb{E}\left[N_{i} \mid T_{\boldsymbol{x} \boldsymbol{y}}\right]= & \sum_{i=2}^{2^{N R} \operatorname{Pr}}[I(\boldsymbol{x}(i) ; \boldsymbol{y}) \geq R \\
& \left.\quad+\max _{j \notin \mathcal{M} \backslash\{i\}} F(I(\boldsymbol{x}(j) ; \boldsymbol{y})-R)\right] \\
= & \sum_{i=2}^{2^{N R} \operatorname{Pr}}\left[Z_{i} \geq \max _{j \notin \mathcal{M} \backslash\{i\}} F\left(Z_{j}\right)\right] \\
\leq & \sum_{i=2}^{2^{N R}} \operatorname{Pr}\left[Z_{i} \geq F\left(z_{1}\right)\right] \\
= & \left(2^{N R}-1\right) \operatorname{Pr}\left[Z_{2} \geq F\left(z_{1}\right)\right]
\end{aligned}
$$

$$
\begin{aligned}
& \stackrel{(\mathrm{a})}{=}\left(2^{N R}-1\right) \\
& \quad \times \operatorname{Pr}\left[I\left(\boldsymbol{x}^{\prime} ; \boldsymbol{y}\right) \geq R+F(I(\boldsymbol{x} ; \boldsymbol{y})-R)\right] \\
& \stackrel{(\mathrm{b})}{\doteq} 2^{N R} 2^{-N[R+F(I(\boldsymbol{x} ; \boldsymbol{y})-R)]} \\
& =2^{-N F(I(\boldsymbol{x} ; \boldsymbol{y})-R)}
\end{aligned}
$$

where in (a), $\boldsymbol{x}^{\prime}$ is drawn independently of $\boldsymbol{y}$ and uniformly over the type class $T_{\boldsymbol{x}}$; and (b) is obtained by application of (B.4).

Averaging over $\boldsymbol{y}$, we obtain

$$
\begin{aligned}
\mathbb{E}\left[N_{i} \mid T_{\boldsymbol{x}}\right] & =\sum_{T_{\boldsymbol{y} \mid \boldsymbol{x}}} \operatorname{Pr}\left[T_{\boldsymbol{y} \mid \boldsymbol{x}}\right] \mathbb{E}\left[N_{i} \mid T_{\boldsymbol{x} \boldsymbol{y}}\right] \\
& \doteq \max _{T_{\boldsymbol{y} \mid \boldsymbol{x}}} \operatorname{Pr}\left[T_{\boldsymbol{y} \mid \boldsymbol{x}}\right] \mathbb{E}\left[N_{i} \mid T_{\boldsymbol{x} \boldsymbol{y}}\right] \\
& \stackrel{(\mathrm{a})}{\leq} \max _{p_{\boldsymbol{y} \mid \boldsymbol{x}}} \exp _{2}\left\{-N\left[D\left(p_{\boldsymbol{y} \mid \boldsymbol{x}} \| p_{Y \mid X} \mid p_{\boldsymbol{x}}\right)\right.\right. \\
& \quad+F(I(\boldsymbol{x} ; \boldsymbol{y})-R)]\} \\
& \stackrel{\text { (b) }}{=} \exp _{2}\left\{-N E_{r, F, N}\left(R, p_{\boldsymbol{x}}, p_{Y \mid X}\right)\right\} \\
& \stackrel{\text { (c) }}{=} \exp _{2}\left\{-N E_{r, F}\left(R, p_{X}, p_{Y \mid X}\right)\right\}
\end{aligned}
$$

where (a) follows from (B.5) and (B.7), (b) from (B.1), and (c) from (B.3). This proves (4.8).

Erasure: The decoder fails to return the transmitted codeword $\boldsymbol{x}=\boldsymbol{x}(1)$ if

$$
I(\boldsymbol{x} ; \boldsymbol{y}) \leq R+\max _{2 \leq i \leq 2^{N R}} F(I(\boldsymbol{x}(i) ; \boldsymbol{y})-R) .
$$

Denote by $p_{\emptyset}\left(p_{\boldsymbol{x}}\right)$ the probability of this event. The event is the disjoint union of events $\mathcal{E}_{1}$ and $\mathcal{E}_{2}$ below. The first one is

$$
\mathcal{E}_{1}: I(\boldsymbol{x} ; \boldsymbol{y}) \leq R+F(0) .
$$

Since $F^{-1}$ is increasing, $\mathcal{E}_{1}$ is equivalent to $F^{-1}(I(\boldsymbol{x} ; \boldsymbol{y})-R) \leq$ 0 . The second event is

$$
\begin{aligned}
\mathcal{E}_{2} & : R+F(0)<I(\boldsymbol{x} ; \boldsymbol{y}) \\
& \leq R+\max _{2 \leq i \leq 2^{N R}} F(I(\boldsymbol{x}(i) ; \boldsymbol{y})-R) \\
& =R+F\left(\max _{2 \leq i \leq 2^{N R}} I(\boldsymbol{x}(i) ; \boldsymbol{y})-R\right)
\end{aligned}
$$

where equality holds because $F$ is nondecreasing. Thus, $\mathcal{E}_{2}$ is equivalent to

$$
\begin{aligned}
\max _{2 \leq i \leq 2^{N R}} I(\boldsymbol{x}(i) ; \boldsymbol{y}) & \geq R+F^{-1}(I(\boldsymbol{x} ; \boldsymbol{y})-R) \\
& >R
\end{aligned}
$$

where the last inequality follows from the fact that $I(\boldsymbol{x} ; \boldsymbol{y})-R>$ $F(0)$ under $\mathcal{E}_{2}$.

Applying (B.6) and (B.3) successively, we have

$$
\begin{aligned}
\operatorname{Pr}\left[\mathcal{E}_{1}\right] & =p_{\emptyset}^{(1)}\left(p_{\boldsymbol{x}}\right) \\
& \triangleq \operatorname{Pr}[I(\boldsymbol{x} ; \boldsymbol{y}) \leq R+F(0)] \\
& \doteq \exp _{2}\left\{-N E_{s p, N}\left(R+F(0), p_{\boldsymbol{x}}, p_{Y \mid X}\right)\right\} \\
& \doteq \exp _{2}\left\{-N E_{\mathrm{sp}}\left(R+F(0), p_{\boldsymbol{x}}, p_{Y \mid X}\right)\right\} .
\end{aligned}
$$


Clearly $p_{\emptyset}\left(p_{\boldsymbol{x}}\right) \sim p_{\emptyset}^{(1)}\left(p_{\boldsymbol{x}}\right) \sim 1$ if

$$
R+F(0) \geq I\left(p_{\boldsymbol{x}}, p_{Y \mid X}\right) .
$$

Next, the probability of $\mathcal{E}_{2}$ conditioned on $\boldsymbol{y}$ is

$$
\begin{aligned}
p_{\emptyset}^{(2)}\left(T_{\boldsymbol{y} \mid \boldsymbol{x}}\right)= & \operatorname{Pr}\left[\max _{2 \leq i \leq 2^{N R}} I(\boldsymbol{x}(i) ; \boldsymbol{y}) \geq R\right. \\
& \left.\quad+F^{-1}(I(\boldsymbol{x} ; \boldsymbol{y})-R)\right] \\
& \stackrel{(\mathrm{a})}{\leq} 2^{N R} \operatorname{Pr}\left[I\left(\boldsymbol{x}^{\prime} ; \boldsymbol{y}\right) \geq R+F^{-1}(I(\boldsymbol{x} ; \boldsymbol{y})-R)\right] \\
& \stackrel{(\mathrm{b})}{\doteq} 2^{-N F^{-1}(I(\boldsymbol{x} ; \boldsymbol{y})-R)}
\end{aligned}
$$

where (a) follows from the union bound, and $\boldsymbol{x}^{\prime}$ is drawn independently of $\boldsymbol{x}$ and $\boldsymbol{y}$ and uniformly from the type class $T_{\boldsymbol{x}}$; and (b) follows from (B.4).

Averaging over $y$, we obtain (B.13) at the bottom of the page, where (a) follows from (B.5) and (B.12) and (b) from the same continuity argument that was used to establish (B.2). Note that $F^{-1}\left(I\left(p_{\boldsymbol{x}}, \tilde{p}_{Y \mid X}\right)-R\right)=0$ for $I\left(p_{\boldsymbol{x}}, \tilde{p}_{Y \mid X}\right)=R+F(0)$. In view of the comment following (B.11), we need to bound $p_{\emptyset}^{(2)}\left(p_{\boldsymbol{x}}\right)$ only in the nontrivial case $R+F(0) \leq I\left(p_{\boldsymbol{x}}, p_{Y \mid X}\right)$. Then, analogously to (A.2), we have

$\max _{\tilde{p}_{Y \mid X}: I\left(p_{\boldsymbol{x}}, \tilde{p}_{Y \mid X}\right) \leq R+F(0)} \exp _{2}\left\{-N D\left(\tilde{p}_{Y \mid X} \| p_{Y \mid X} \mid p_{\boldsymbol{x}}\right)+0\right\}$

$=\exp _{2}\left\{-N E_{\mathrm{sp}}\left(R+F(0), p_{\boldsymbol{x}}, p_{Y \mid X}\right)\right\}$

$=\max _{\tilde{p}_{Y \mid X}: I\left(p_{\boldsymbol{x}}, \tilde{p}_{Y \mid X}\right)=R+F(0)} \exp _{2}\left\{-N D\left(\tilde{p}_{Y \mid X} \| p_{Y \mid X} \mid p_{\boldsymbol{x}}\right)+0\right\}$.

Thus, (B.13) reduces to the unconstrained maximum

$$
\begin{aligned}
& p_{\emptyset}^{(2)}\left(p_{\boldsymbol{x}}\right) \doteq \max _{\tilde{p}_{Y \mid X}} \exp _{2}\left\{-N\left[D\left(\tilde{p}_{Y \mid X} \| p_{Y \mid X} \mid p_{\boldsymbol{x}}\right)\right.\right. \\
&\left.\left.\quad+\left|F^{-1}\left(I\left(p_{\boldsymbol{x}}, \tilde{p}_{Y \mid X}\right)-R\right)\right|^{+}\right]\right\} \\
&=\exp _{2}\left\{-N E_{r,\left|F^{-1}\right|^{+}}\left(R, p_{\boldsymbol{x}}, p_{Y \mid X}\right)\right\} .
\end{aligned}
$$

Since $\mathcal{E}_{1}$ and $\mathcal{E}_{2}$ are disjoint events, we obtain

$$
\begin{gathered}
p_{\emptyset}\left(p_{\boldsymbol{x}}\right)=p_{\emptyset}^{(1)}\left(p_{\boldsymbol{x}}\right)+p_{\emptyset}^{(2)}\left(p_{\boldsymbol{x}}\right) \\
\doteq \exp _{2}\left\{-N \min \left\{E_{\mathrm{sp}}\left(R+F(0), p_{X}, p_{Y \mid X}\right),\right.\right. \\
\left.\left.E_{r,\left|F^{-1}\right|^{+}}\left(R, p_{X}, p_{Y \mid X}\right)\right\}\right\} .
\end{gathered}
$$

The function $F^{-1}(t)$ has a zero at $t=F(0)$. Applying (4.7), we obtain

$$
\begin{aligned}
& E_{r,\left|F^{-1}\right|^{+}}\left(R, p_{X}, p_{Y \mid X}\right) \\
& \quad=\min _{R^{\prime}}\left[E_{\mathrm{sp}}\left(R^{\prime}, p_{X}, p_{Y \mid X}\right)+\left|F^{-1}\left(R^{\prime}-R\right)\right|^{+}\right] \\
& \quad \leq E_{\mathrm{sp}}\left(R+F(0), p_{X}, p_{Y \mid X}\right)+0 .
\end{aligned}
$$

Hence

$$
p_{\emptyset}\left(p_{\boldsymbol{x}}\right) \dot{\leq} \exp _{2}\left\{-N E_{r,\left|F^{-1}\right|^{+}}\left(R, p_{X}, p_{Y \mid X}\right)\right\},
$$

which proves (4.9).

\section{APPENDIX C \\ PROOF OF PROPOSITION 5.1}

We first prove (5.11). Recall (4.7) and (4.6), restated here for convenience:

$$
\begin{aligned}
E_{r, F}\left(R, p_{X}, \mathcal{W}\right) & =\min _{R^{\prime}}\left[E_{\mathrm{sp}}\left(R^{\prime}, p_{X}, \mathcal{W}\right)+F\left(R^{\prime}-R\right)\right] \\
E_{\mathrm{sp}}\left(R, p_{X}, \mathcal{W}\right) & \equiv E_{\mathrm{sp}}\left(R^{\prime}, p_{X}, \mathcal{W}\right)+F_{R, p_{X}, \mathcal{W}}\left(R^{\prime}-R\right),
\end{aligned}
$$

$\forall R^{\prime}$.

\section{Hence}

$$
E_{r, F_{R, p_{X}, \mathcal{W}}}\left(R, p_{X}, \mathcal{W}\right)=E_{\mathrm{sp}}\left(R, p_{X}, \mathcal{W}\right) .
$$

We have

$$
\begin{aligned}
& \alpha \stackrel{(\mathrm{a})}{=} \Delta+E_{\mathrm{sp}}\left(R, p_{X}, \mathcal{W}\right) \\
& \stackrel{\text { b) }}{=} \Delta+E_{r, F_{R, p_{X}, \mathcal{W}}}\left(R, p_{X}, \mathcal{W}\right) \\
& \stackrel{(\mathrm{c})}{=} E_{r, \Delta+F_{R, p_{X}}, \mathcal{W}}\left(R, p_{X}, \mathcal{W}\right) \\
& \stackrel{(\mathrm{d})}{=} E_{F^{L *}}\left(R, p_{X}, \mathcal{W}\right)
\end{aligned}
$$

where (a) follows from (5.9) (b) from (C.1), (c) from the definition (4.3) of $E_{r, F}(\cdot)$, and (d) from the definition (5.11) of $F^{L *}$. The feasible set $\mathcal{F}^{L}\left(R, p_{X}, \mathcal{W}, \alpha\right)$ defined in (5.5) takes the form

$$
\begin{aligned}
\mathcal{F}^{L}\left(R, p_{X}, \mathcal{W}, \alpha\right)= & \left\{F: E_{r, F}\left(R, p_{X}, \mathcal{W}\right) \geq \alpha\right\} \\
= & \left\{F: E_{r, F}\left(R, p_{X}, \mathcal{W}\right)\right. \\
& \left.\geq E_{r, F^{L *}}\left(R, p_{X}, \mathcal{W}\right)\right\} \\
= & \left\{F: F \succeq F^{L *}\right\}
\end{aligned}
$$

$$
\begin{aligned}
\operatorname{Pr}\left[\mathcal{E}_{2}\right]=p_{\emptyset}^{(2)}\left(p_{\boldsymbol{x}}\right) & \triangleq \sum_{T_{\boldsymbol{y} \mid \boldsymbol{x}}: I(\boldsymbol{x} ; \boldsymbol{y})>R+F(0)} \operatorname{Pr}\left[T_{\boldsymbol{y} \mid \boldsymbol{x}}\right] p_{\emptyset}^{(2)}\left(T_{\boldsymbol{y} \mid \boldsymbol{x}}\right) \\
& \doteq \max _{T_{\boldsymbol{y} \mid \boldsymbol{x}}: I(\boldsymbol{x} ; \boldsymbol{y}) \geq R+F(0)} \operatorname{Pr}\left[T_{\boldsymbol{y} \mid \boldsymbol{x}}\right] p_{\emptyset}^{(2)}\left(T_{\boldsymbol{y} \mid \boldsymbol{x}}\right) \\
& \stackrel{(\mathrm{a})}{\doteq} \max _{p_{\boldsymbol{y} \mid \boldsymbol{x}}: I(\boldsymbol{x} ; \boldsymbol{y}) \geq R+F(0)} \exp _{2}\left\{-N\left[D\left(p_{\boldsymbol{y} \mid \boldsymbol{x}} \| p_{Y \mid X} \mid p_{\boldsymbol{x}}\right)+F^{-1}(I(\boldsymbol{x} ; \boldsymbol{y})-R)\right]\right\} \\
& \stackrel{(\mathrm{b})}{\doteq} \operatorname{pr}_{Y \mid X} \max _{\left.\tilde{p}_{Y}: \tilde{p}_{\boldsymbol{x}}, \tilde{p}_{Y \mid X}\right) \geq R+F(0)} \exp _{2}\left\{-N\left[D\left(\tilde{p}_{Y \mid X} \| p_{Y \mid X} \mid p_{\boldsymbol{x}}\right)+F^{-1}\left(I\left(p_{\boldsymbol{x}}, \tilde{p}_{Y \mid X}\right)-R\right)\right]\right\}
\end{aligned}
$$


where the last line follows from the monotonicity property of Proposition 4.1(ii). As indicated below the statement of Proposition 5.1, this implies $F^{L *}$ achieves the supremum in (5.5).

The alternative expression (5.12) for $F^{L *}$ is obtained by substituting (4.5) and (5.9) into (5.11).

To prove (5.13), we simply observe that if $F^{L *} \preceq F$ for all $F \in \mathcal{F}^{L}\left(R, p_{X}, \mathcal{W}, \alpha\right)$, then $F^{*} \preceq F$ for all $F \in$ $\mathcal{F}\left(R, p_{X}, \mathcal{W}, \alpha\right)$, where $F^{*}(t)=\max \left(t, F^{L *}(t)\right)$.

\section{APPENDIX D}

\section{PROOF OF PROPOSITION 5.3}

We have

$$
\begin{aligned}
& E_{\emptyset}^{L}\left(R, p_{X}, \mathcal{W}, \alpha\right) \stackrel{(\mathrm{a})}{=} E_{r,\left|\left(F^{L *}\right)^{-1}\right|^{+}}\left(R, p_{X}, \mathcal{W}\right) \\
& \stackrel{(\mathrm{b})}{=} \min _{R^{\prime}}\left[E_{\mathrm{sp}}\left(R^{\prime}, p_{X}, \mathcal{W}\right)\right. \\
&\left.\quad+\left|\left(F^{L *}\right)^{-1}\right|^{+}\left(R^{\prime}-R\right)\right]
\end{aligned}
$$

where equality (a) results from Propositions 4.2 and 5.1, and (b) from (4.5). From (5.11) and property (P3) in Section III, we obtain the inverse function

$$
\left(F^{L *}\right)^{-1}(t)=F_{R, p_{X}, \mathcal{W}}^{-1}(t-\Delta)
$$

where $F_{R, p_{X}, \mathcal{W}}(t)$ is given in (4.5). We have $\left(F^{L *}\right)^{-1}(0)=0$ and thus $\left|\left(F^{L *}\right)^{-1}(t)\right|^{+}=0$ for all $t \leq 0$. Since the function $E_{\mathrm{sp}}\left(R^{\prime}, p_{X}, \mathcal{W}\right)$ is nonincreasing in $R^{\prime}$, we may write (D.1) as

$$
\begin{aligned}
& E_{\emptyset}^{L}\left(R, p_{X}, \mathcal{W}, \alpha\right)= \\
& \min _{R^{\prime} \geq R+\Delta}[\underbrace{E_{\mathrm{sp}}\left(R^{\prime}, p_{X}, \mathcal{W}\right)+F_{R, p_{X}, \mathcal{W}}^{-1}\left(R^{\prime}-R-\Delta\right)}_{h\left(R^{\prime}\right)}] . \quad \text { (D.3) }
\end{aligned}
$$

By assumption, $E_{\mathrm{sp}}\left(R, p_{X}, \mathcal{W}\right)$ is convex in $R$, and therefore $F_{R, p_{X}, \mathcal{W}}(t)$ is concave. By application of Property (P5) in Section III, the function $F_{R, p_{X}, \mathcal{W}}^{-1}$ is convex, and thus so is $h\left(R^{\prime}\right)$ in (D.3). The derivative of $F_{R, p_{X}, \mathcal{W}}^{-1}$ is given by

$$
\begin{aligned}
\left(F_{R, p_{X}, \mathcal{W}}^{-1}\right)^{\prime}(t) & \stackrel{(\mathrm{a})}{=} \frac{1}{F_{R, p_{X}, \mathcal{W}}^{\prime}(t)} \\
& \stackrel{(\mathrm{b})}{=} \frac{1}{-E_{\mathrm{sp}}^{\prime}\left(R+t, p_{X}, \mathcal{W}\right)}
\end{aligned}
$$

where (a) follows from Property (P4) and (b) from (4.6). Differentiating $h\left(R^{\prime}\right)$ defined in (D.3) and using (D.4) we obtain

$$
h^{\prime}\left(R^{\prime}\right)=E_{\mathrm{sp}}^{\prime}\left(R^{\prime}, p_{X}, \mathcal{W}\right)+\frac{1}{-E_{\mathrm{sp}}^{\prime}\left(R^{\prime}-\Delta, p_{X}, \mathcal{W}\right)} .
$$

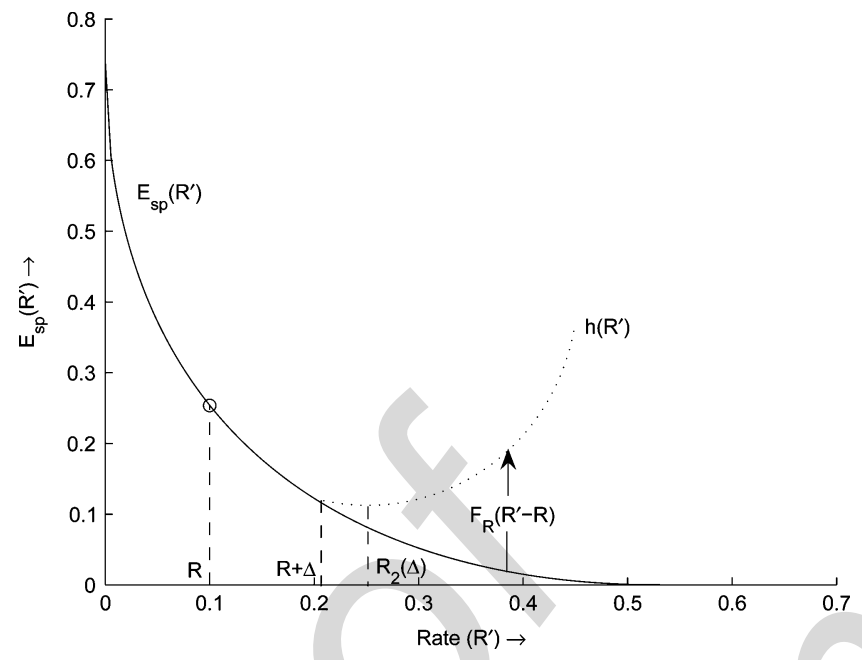

Fig. 3. Construction and minimization of $h\left(R^{\prime}\right)$ for Case (i).

By Proposition 5.1 and the definition of $\Delta$ in (5.9), we have

$$
E_{i}\left(R, p_{X}, \mathcal{W}\right)=\alpha=E_{\mathrm{sp}}\left(R, p_{X}, \mathcal{W}\right)+\Delta
$$

Next we prove the statements (i)-(iv).

(i) It is assumed that

$$
\max \left(0, R^{\operatorname{conj}}\left(p_{X}, \mathcal{W}\right)-R\right) \leq \Delta \leq I\left(p_{X}, \mathcal{W}\right)-R .
$$

This case is illustrated in Fig. 3. We have

$$
R+\Delta \geq \max \left(R, R^{\operatorname{conj}}\left(p_{X}, \mathcal{W}\right)\right) \geq R_{c r}\left(p_{X}, \mathcal{W}\right) .
$$

Hence from (D.5) we have (D.7) shown at the bottom of the page. By convexity of $h(\cdot)$, this implies that $R+\Delta$ minimizes $h\left(R^{\prime}\right)$ over $R^{\prime} \geq R+\Delta$, and so

$$
E_{\emptyset}^{L}\left(R, p_{X}, \mathcal{W}, \alpha\right)=h(R+\Delta)=E_{\mathrm{sp}}\left(R+\Delta, p_{X}, \mathcal{W}\right) .
$$

(ii) Due to (D.6), we have either $R \geq R_{c r}\left(p_{X}, \mathcal{W}\right)$ or $R+\Delta \geq R^{\operatorname{conj}}\left(p_{X}, \mathcal{W}\right) \geq R$. In both cases

$$
-E_{\mathrm{sp}}^{\prime}\left(R, p_{X}, \mathcal{W}\right) \leq \frac{1}{-E_{\mathrm{sp}}^{\prime}\left(R+\Delta, p_{X}, \mathcal{W}\right)} .
$$

Let $F(t)=\Delta+\lambda|t|^{+}$where $\lambda$ is sandwiched by the left and right sides of the above inequality. We have $F^{\prime}(t)=\lambda \mathbb{1}_{\{t \geq 0\}}$. The inverse function is $F^{-1}(t)=\frac{1}{\lambda}(t-\Delta)$ for $t \geq \Delta$. Hence

$$
\begin{aligned}
h^{\prime}(R+\Delta) & =E_{\mathrm{sp}}^{\prime}\left(R+\Delta, p_{X}, \mathcal{W}\right)+\frac{1}{-E_{\mathrm{sp}}^{\prime}\left(R, p_{X}, \mathcal{W}\right)} \\
& \geq \begin{cases}E_{\mathrm{sp}}^{\prime}\left(R, p_{X}, \mathcal{W}\right)+\frac{1}{-E_{\mathrm{sp}}^{\prime}\left(R, p_{X}, \mathcal{W}\right)} \geq 0 & : \text { if } R \geq R_{c r}\left(p_{X}, \mathcal{W}\right) \\
E_{\mathrm{sp}}^{\prime}\left(R^{\operatorname{conj}}\left(p_{X}, \mathcal{W}\right), p_{X}, \mathcal{W}\right)+\frac{1}{-E_{\mathrm{sp}}^{\prime}\left(R, p_{X}, \mathcal{W}\right)}=0 & : \text { if } R \leq R_{c r}\left(p_{X}, \mathcal{W}\right)\end{cases} \\
& \geq 0 .
\end{aligned}
$$




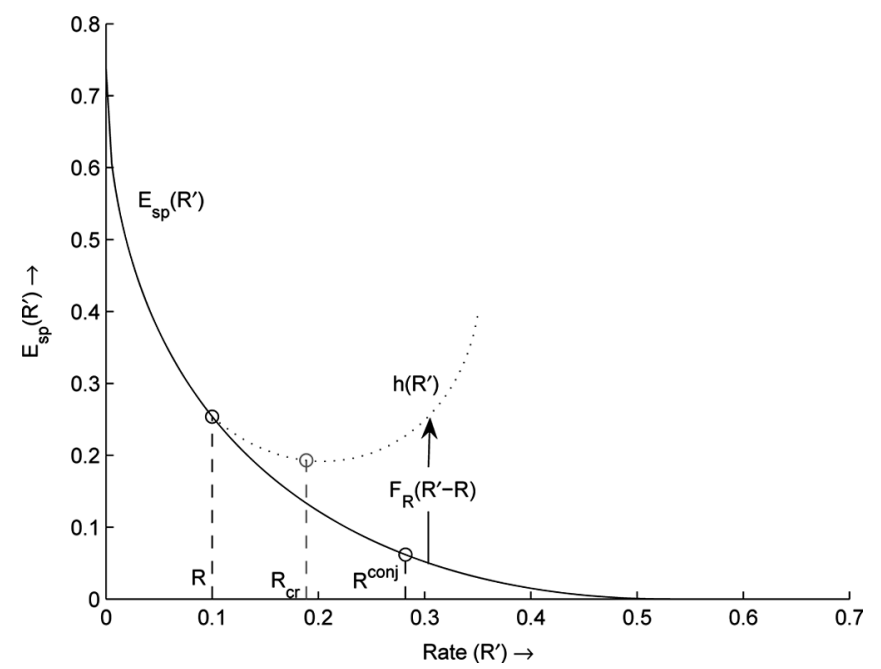

Fig. 4. Construction and minimization of $h\left(R^{\prime}\right)$ for Case (ii), with $\Delta=0$.

$\left(F^{-1}\right)^{\prime}(t)=\frac{1}{\lambda}$ for $t \geq \Delta$. Substituting $F$ and $F^{-1}$ into (4.7), we obtain

$$
\begin{aligned}
& E_{r, F}\left(R, p_{X}, \mathcal{W}\right) \\
& \quad=\min _{R^{\prime} \geq R}\left[E_{\mathrm{sp}}\left(R^{\prime}, p_{X}, \mathcal{W}\right)+\Delta+\lambda\left(R^{\prime}-R\right)\right] \\
& E_{r,\left|F^{-1}\right|^{+}}\left(R, p_{X}, \mathcal{W}\right) \\
& \quad=\min _{R^{\prime} \geq R+\Delta}\left[E_{\mathrm{sp}}\left(R^{\prime}, p_{X}, \mathcal{W}\right)+\frac{1}{\lambda}\left(R^{\prime}-R-\Delta\right)\right] .
\end{aligned}
$$

Taking derivatives of the bracketed terms with respect to $R^{\prime}$ and recalling that

$$
\lambda \geq-E_{\mathrm{sp}}^{\prime}\left(R, p_{X}, \mathcal{W}\right), \quad \frac{1}{\lambda} \geq-E_{\mathrm{sp}}^{\prime}\left(R+\Delta, p_{X}, \mathcal{W}\right)
$$

we observe that these derivatives are nonnegative. Since $E_{\mathrm{sp}}\left(\cdot, p_{X}, \mathcal{W}\right)$ is convex, the minima are achieved at $R$ and $R+\Delta$, respectively.

The resulting exponents are $E_{\mathrm{sp}}\left(R, p_{X}, \mathcal{W}\right)+\Delta$ and $E_{\mathrm{sp}}\left(R+\Delta, p_{X}, \mathcal{W}\right)$ which coincide with the optimal exponents of (5.14).

(iii) $R \leq R_{c r}\left(p_{X}, \mathcal{W}\right)$ and $0 \leq \Delta \leq R^{\operatorname{conj}}\left(p_{X}, \mathcal{W}\right)-R$. This case is illustrated in Fig. 4 in the case $\Delta=0$. From (D.7), we have $h^{\prime}\left(R^{\prime}\right)=0$ if and only if $R^{\prime}$ and $R^{\prime}-\Delta$ are conjugate rates. In this case, using the above assumption on $\Delta$, we have

$$
\begin{aligned}
R \leq R^{\prime}-\Delta & =R_{1}(\Delta) \leq R_{c r}\left(p_{X}, \mathcal{W}\right) \leq R^{\prime} \\
& =R_{2}(\Delta) \leq R^{\operatorname{conj}}\left(p_{X}, \mathcal{W}\right) .
\end{aligned}
$$

Hence, $R_{2}(\Delta)=R^{\prime} \geq R+\Delta$ is feasible for (D.3) and minimizes $h(\cdot)$. Substituting $R^{\prime}$ back into (D.3), we obtain

$$
\begin{aligned}
E_{\emptyset}^{L}\left(R, p_{X}, \mathcal{W}, \alpha\right)= & h\left(R_{2}(\Delta)\right) \\
= & E_{\mathrm{sp}}\left(R_{2}(\Delta), p_{X}, \mathcal{W}\right) \\
& +F_{R, p_{X}, \mathcal{W}}^{-1}\left(R_{1}(\Delta)-R\right)
\end{aligned}
$$

which establishes (5.16).

(iv) $R \leq R_{c r}\left(p_{X}, \mathcal{W}\right)$ and $R_{\infty}\left(p_{X}, \mathcal{W}\right)-R \leq \Delta \leq 0$.
Again we have $h^{\prime}\left(R^{\prime}\right)=0$ if and only if $R^{\prime}$ and $R^{\prime}-\Delta$ are conjugate rates. Then, using the above assumption on $\Delta$, we have

$$
R \leq R^{\prime}=R_{1}(\Delta) \leq R_{c r}\left(p_{X}, \mathcal{W}\right) \leq R^{\prime}-\Delta=R_{2}(\Delta)
$$

Hence $R_{1}(\Delta)=R^{\prime} \geq R+\Delta$ is feasible for (D.3) and minimizes $h(\cdot)$. Substituting $R^{\prime}$ back into (D.3), we obtain

$$
\begin{aligned}
E_{\emptyset}^{L}\left(R, p_{X}, \mathcal{W} \alpha\right)= & h\left(R_{1}(\Delta)\right) \\
= & E_{\mathrm{sp}}\left(R_{1}(\Delta), p_{X}, \mathcal{W}\right) \\
& +F_{R, p_{X}, \mathcal{W}}^{-1}\left(R_{2}(\Delta)-R\right)
\end{aligned}
$$

which establishes (5.17).

\section{APPENDIX E}

PROOF OF LEMMA 5.4

First we prove (5.20). For any $R_{0}<R_{1}$, we have

$$
\begin{aligned}
\int_{R_{0}}^{R_{1}} & \underline{E_{\mathrm{sp}}^{\prime}}\left(R, p_{X}, \mathcal{W}\right) d R \\
& \stackrel{(\mathrm{a})}{=} \int_{R_{0}}^{R_{1}} \min _{p_{Y \mid X} \in \mathcal{W}} E_{\mathrm{sp}}^{\prime}\left(R, p_{X}, p_{Y \mid X}\right) d R \\
& \leq \min _{p_{Y \mid X} \in \mathcal{W}} \int_{R_{0}}^{R_{1}} E_{\mathrm{sp}}^{\prime}\left(R, p_{X}, p_{Y \mid X}\right) d R \\
& =\min _{p_{Y \mid X} \in \mathcal{W}}\left[E_{\mathrm{sp}}\left(R_{1}, p_{X}, p_{Y \mid X}\right)-E_{\mathrm{sp}}\left(R_{0}, p_{X}, p_{Y \mid X}\right)\right] \\
& \stackrel{\mathrm{b})}{\leq} E_{\mathrm{sp}}\left(R_{1}, p_{X}, p_{Y \mid X}^{*}\right)-E_{\mathrm{sp}}\left(R_{0}, p_{X}, p_{Y \mid X}^{*}\right) \\
& =\min _{p_{Y \mid X} \in \mathcal{W}} E_{\mathrm{sp}}\left(R_{1}, p_{X}, p_{Y \mid X}\right)-E_{\mathrm{sp}}\left(R_{0}, p_{X}, p_{Y \mid X}^{*}\right) \\
& \leq \min _{p_{Y \mid X} \in \mathcal{W}} E_{\mathrm{sp}}\left(R_{1}, p_{X}, p_{Y \mid X}\right)-\min _{p_{Y \mid X} \in \mathcal{W}} E_{\mathrm{sp}}\left(R_{0}, p_{X}, p_{Y \mid X}\right) \\
& =E_{\mathrm{sp}}\left(R_{1}, p_{X}, \mathcal{W}\right)-E_{\mathrm{sp}}\left(R_{0}, p_{X}, \mathcal{W}\right) \\
& =\int_{R_{0}}^{R_{1}} E_{\mathrm{sp}}^{\prime}\left(R, p_{X}, \mathcal{W}\right) d R
\end{aligned}
$$

where (a) follows from the definition of $\underline{E}_{\mathrm{sp}}^{\prime}$ in (5.18), and we choose $p_{Y \mid X}^{*}$ in inequality (b) as the minimizer of $E_{\mathrm{sp}}\left(R_{1}, p_{X}, \cdot\right)$ over $\mathcal{W}$. Since (E.1) holds for all $R_{0}<R_{1}$, we must have inequality between the integrands in the left and right sides: $\underline{E}_{\mathrm{sp}}^{\prime}\left(R, p_{X}, \mathcal{W}\right) \leq E_{\mathrm{sp}}^{\prime}\left(R, p_{X}, \mathcal{W}\right)$. Moreover, the three inequalities used to derive (E.1) hold with equality if the same $p_{Y \mid X}^{* *}$ minimizes $E_{\mathrm{sp}}^{\prime}\left(R, p_{X}, \cdot\right)$ at all rates, and the same $p_{Y \mid X}^{*}$ minimizes $E_{\mathrm{sp}}\left(R, p_{X}, \cdot\right)$ at all rates. We need not (and generally do not) have $p_{Y \mid X}^{* *}=p_{Y \mid X}^{*} \cdot{ }^{5}$

Next we prove (5.21). By definition of $R^{\operatorname{conj}}\left(p_{X}, \mathcal{W}\right)$, we have

$$
\begin{gathered}
-E_{\mathrm{sp}}^{\prime}\left(R^{\mathrm{conj}}\left(p_{X}, \mathcal{W}\right), p_{X}, \mathcal{W}\right) \\
=\frac{1}{-E_{\mathrm{sp}}^{\prime}\left(R, p_{X}, \mathcal{W}\right)} \\
\quad \stackrel{(\mathrm{a})}{\geq} \frac{1}{-\underline{E}_{\mathrm{sp}}^{\prime}\left(R, p_{X}, \mathcal{W}\right)}
\end{gathered}
$$

${ }^{5}$ While $p_{Y \mid X}^{*}$ is the noisiest channel in $\mathcal{W}, p_{Y \mid X}^{* *}$ may be the cleanest channel in $\mathcal{W}$, as in the BSC example of Section VII. 


$$
\begin{aligned}
& =\min _{p_{Y \mid X} \in \mathcal{W}} \frac{1}{-E_{\mathrm{sp}}^{\prime}\left(R, p_{X}, p_{Y \mid X}\right)} \\
& =\min _{p_{Y \mid X} \in \mathcal{W}}\left[-E_{\mathrm{sp}}^{\prime}\left(R^{\mathrm{conj}}\left(p_{X}, p_{Y \mid X}\right), p_{X}, p_{Y \mid X}\right)\right] \\
& \stackrel{(\mathrm{b})}{\geq} \min _{p_{Y \mid X} \in \mathcal{W}}\left[-E_{\mathrm{sp}}^{\prime}\left(\bar{R}^{\mathrm{conj}}\left(p_{X}, \mathcal{W}\right), p_{X}, p_{Y \mid X}\right)\right] \\
& \stackrel{(\mathrm{c})}{=}-\underline{E}_{\mathrm{sp}}^{\prime}\left(\bar{R}^{\mathrm{conj}}\left(p_{X}, \mathcal{W}\right), p_{X}, \mathcal{W}\right) \\
& \stackrel{(\mathrm{d})}{\geq}-E_{\mathrm{sp}}^{\prime}\left(\bar{R}^{\mathrm{conj}}\left(p_{X}, \mathcal{W}\right), p_{X}, \mathcal{W}\right)
\end{aligned}
$$

where (a) and (d) are due to (5.20), (b) to the definition of $\bar{R}^{\mathrm{conj}}\left(p_{X}, \mathcal{W}\right)$ in (5.19) and the fact that $-E_{\mathrm{sp}}^{\prime}\left(R, p_{X}, p_{Y \mid X}\right)$ is a decreasing function of $R$, and (c) from (5.18). Since $-E_{\mathrm{sp}}^{\prime}\left(R, p_{X}, \mathcal{W}\right)$ is also a decreasing function of $R$, we must have $R^{\text {conj }}\left(p_{X}, \mathcal{W}\right) \leq \bar{R}^{\text {conj }}\left(p_{X}, \mathcal{W}\right)$. Moreover, the conditions for equality are the same as those for equality in (5.20).

\section{ACKNOWLEDGMENT}

The author wishes to thank Shankar Sadasivam for numerical evaluation of the error exponent formulas in Section VII, Prof. Lizhong Zheng for helpful comments, and the reviewers for their careful reading of the manuscript and recommended improvements.

\section{REFERENCES}

[1] I. Csiszár and J. Körner, Information Theory: Coding Theory for Discrete Memoryless Systems. New York: Academic, 1981.

[2] A. Lapidoth and P. Narayan, "Reliable communication under channel uncertainty," IEEE Trans. Inf. Theory, vol. 44, no. 6, pp. 2148-2177, Oct. 1998.

[3] G. D. Forney, Jr, "Exponential error bounds for erasure, list, and decision feedback schemes," IEEE Trans. Inf. Theory, vol. IT-14, no. 2, pp. 206-220, Mar. 1968.

[4] I. E. Telatar, "Multi-Access Communications With Decision Feedback Decoding," Ph.D. dissertation, MIT, Cambridge, MA, 1992.
[5] I. E. Telatar and R. G. Gallager, "New exponential upper bounds to error and erasure probabilities," in Proc. IEEE Int. Symp. Information Theory (ISIT'94), Trondheim, Norway, Jun. 1994, p. 379.

[6] N. Merhav and M. Feder, "Minimax universal decoding with erasure option," IEEE Trans. Inf. Theory, vol. 53, no. 5, pp. 1664-1675, May 2007.

[7] S. A. Kassam and H. V. Poor, "Robust techniques for signal processing: A survey," Proc. IEEE, vol. 73, no. 3, pp. 433-481, Mar. 1985.

[8] W. Hoeffding, "Asymptotically optimal tests for multinomial distributions," Ann. Math. Statist., vol. 36, no. 2, pp. 369-400, 1965.

[9] G. Tusnády, "On asymptotically optimal tests," Ann. Statist., vol. 5, no. 2, pp. 385-393, 1977.

[10] O. Zeitouni and M. Gutman, "On universal hypotheses testing via large deviations," IEEE Trans. Inf. Theory, vol. 37, no. 2, pp. 285-290, Mar. 1991.

[11] I. Csiszár, Personal Communication Aug. 2007.

[12] M. Feder and N. Merhav, "Universal composite hypothesis testing: A competitive minimax approach," IEEE Trans. Inf. Theory, vol. 48, no. 6, pp. 1504-1517, Jun. 2002.

Pierre Moulin (S'89-M'90-SM'98-F'03) received the doctoral degree from Washington University, St. Louis, MO, in 1990.

After graduation, he joined at Bell Communications Research, Morristown, NJ, as a Research Scientist. In 1996, he joined the University of Illinois at Urbana-Champaign (UIUC), Urbana, where he is currently Professor in the Department of Electrical and Computer Engineering, Research Professor at the Beckman Institute and the Coordinated Science Laboratory, an affiliate Professor in the Department of Statistics, and Sony Faculty Scholar. His fields of professional interest include image and video processing, compression, statistical signal processing and modeling, media security, decision theory, and information theory.

Dr. Moulin has served on the editorial boards of the IEEE TRANSACTIONS ON INFORMATION THEORY and the IEEE TRANSACTIONS ON IMAGE PROCESSING, He currently serves on the Editorial Boards of PROCEEDINGS OF IEEE and of Foundations and Trends in Signal Processing. He was co-founding Editor-inChief of the IEEE TRANSACTIONS ON INFORMATION FORENSICS AND SECURITY (2005-2008), member of the IEEE Signal Processing Society Board of Governors (2005-2007), and has served IEEE in various other capacities. He received a 1997 CAREER award from the National Science Foundation and an IEEE Signal Processing Society 1997 Senior Best Paper award. He is also coauthor (with Juan Liu) of a paper that received an IEEE Signal Processing Society 2002 Young Author Best Paper award. He was 2003 Beckman Associate of UIUCs Center for Advanced Study and plenary speaker for ICASSP 2006 and several other conferences 